\title{
Enhancing the Nutritional Quality of Major Food Crops Through Conventional and Genomics-Assisted Breeding
}

\author{
Kiran B. Gaikwad ${ }^{1 *}$, Sushma Rani ${ }^{2}$, Manjeet Kumar ${ }^{1}$, Vikas Gupta ${ }^{3}$, Prashanth H. Babu ${ }^{1}$, \\ Naresh Kumar Bainsla ${ }^{1}$ and Rajbir Yadav ${ }^{1}$ \\ 1 Division of Genetics, Indian Council of Agricultural Research (ICAR)-Indian Agricultural Research Institute, New Delhi, India, \\ ${ }^{2}$ Indian Council of Agricultural Research (ICAR)-National Institute for Plant Biotechnology, New Delhi, India, ${ }^{3}$ Division of \\ Genetics, Indian Council of Agricultural Research (ICAR)-Indian Institute of Wheat and Barley Research, Karnal, India
}

Nutritional stress is making over two billion world population malnourished. Either our commercially cultivated varieties of cereals, pulses, and oilseed crops are deficient in essential nutrients or the soils in which these crops grow are becoming devoid of minerals. Unfortunately, our major food crops are poor sources of micronutrients required for normal human growth. To overcome the problem of nutritional deficiency, greater emphasis should be laid on the identification of genes/quantitative trait loci (QTLS) pertaining to essential nutrients and their successful deployment in elite breeding lines through marker-assisted breeding. The manuscript deals with information on identified QTLs for protein content, vitamins, macronutrients, micro-nutrients, minerals, oil content, and essential amino acids in major food crops. These QTLs can be utilized in the development of nutrient-rich crop varieties. Genome editing technologies that can rapidly modify genomes in a precise way and will directly enrich the nutritional status of elite varieties could hold a bright future to address the challenge of malnutrition.

Keywords: nutritional quality, genomics assisted breeding, QTLs, food crops, malnutrition, genome editing, CRISPR/Cas9, plant breeding

Kiran B. Gaikwad

kiran.gaikwad@icar.gov.in

gaikwadkb@gmail.com

Specialty section:

This article was submitted to Nutrigenomics,

a section of the journal

Frontiers in Nutrition

Received: 08 February 2020 Accepted: 03 September 2020 Published: 26 November 2020

Citation:

Gaikwad KB, Rani S, Kumar M

Gupta V, Babu PH, Bainsla NK and Yadav $R$ (2020) Enhancing the

Nutritional Quality of Major Food

Crops Through Conventional and

Genomics-Assisted Breeding.

Front. Nutr. 7:533453

doi: 10.3389/fnut.2020.533453

\section{INTRODUCTION}

Over two billion of the world's population is at the risk of micronutrient deficiency which resulted due to an inadequate supply of micronutrients in daily diet (1). The principal reason behind this nutrient stress is our food crops which are inadequate to supply essential nutrients as they are grown on the soils which are deficient in minerals. The agricultural production scenario in most developing countries does not address the issues related to malnutrition; rather, it focuses on increasing grain yield and crop productivity. Malnutrition is also a vital threat to the population's health and productivity and is reviewed as the largest adverse health impact of climate change. In the last few decades, the climate had altered a lot since the temperature is rising in many parts of the world, coupled with low or unexpectedly high rainfall. According to the World Health Organization and the Fifth Assessment Report of the Intergovernmental Panel on Climate Change, malnutrition is considered as one of the five largest adverse health impacts of climate change (2).

The situation of continuous degrading natural resources, farm resource constraints, and agriculture affected by changing climate in the developing countries is making a rapid rise in micronutrient deficiency in food grains, thereby increasing micronutrient malnutrition among 
the population, but thanks to the innovative breeding efforts by plant breeders, the agriculture in developing countries is shifting toward producing nutrient-rich food crops. This will eliminate micronutrient malnutrition in these countries, where daily diets are dominated by micronutrient-poor staple food crops. Biofortification is a strategy that can overcome nutrient deficiency more sustainably. It is a one-time investment and offers a cost-effective, long-term, and sustainable approach in fighting hidden hunger because, once the biofortified crops are developed, there are no additional costs of buying the fortificants and adding them to the food supply during processing (3). Biofortification is a process of enriching the content of vitamins and minerals in crops through genetic and agronomic interventions. Biofortification not only makes our food nutrientrich but also reduces the cost of external amendments required to make food rich in nutrients. Approaches such as conventional plant breeding, molecular breeding, transgenic techniques, or agronomical practices provide a new avenue for the development of nutrient-rich crops. Biofortification is mainly focused on important diet-dominant foods such as cereal crops, viz., rice, wheat, maize, sorghum, millet, and legumes, and starchy food, viz., potato and sweet potatoes.

We, humans, require around 40 known nutrients in optimum amounts to live a healthy life. These nutrients are classified as macro nutrients, micronutrients, and minerals. The micronutrients, viz., iron, zinc, copper, manganese, iodine, selenium, molybdenum, cobalt, nickel, and vitamin $A$, are extremely important for a wide range of metabolic processes which ultimately lead to normal growth and development. The mineral elements such as sodium, magnesium, calcium, potassium, phosphorous, chlorine, and sulfur are classified as essential nutrients that are required in trace amounts in the body. Overall, these nutrients play a pivotal role in our physical and mental development (4). Apart from micronutrients and minerals, protein and oil content are the chief nutritional factors in food grains. Therefore, it is foremost essence to incorporate essential micronutrients, minerals, and proteins into the diets of resource-poor populations whose diet is based on cereals such as rice, wheat, cassava, and maize which contain insufficient amounts of several nutrients.

The adverse effect of climate change on nutritional food security will be primarily seen in the developing countries of Africa and South Asia. Climate change will affect not only the number of food crops but also nutritional quality. Overall, hundreds of millions of people are expected to be placed at risk of zinc, iron, and/or protein deficiencies as a result of rising $\mathrm{CO}_{2}$ concentrations. Recent studies proved that elevated $\mathrm{CO}_{2}$ concentration in the atmosphere reduces the concentrations of iron, zinc, and proteins in staple cereals, viz., rice, wheat, barley, and pulses. Nowadays, $\mathrm{CO}_{2}$ concentrations of $550 \mathrm{ppm}$, which is higher than normal, can lead to $3-11 \%$ decreases of zinc and iron concentrations in cereal grains and legumes (5). Further increase in $\mathrm{CO}_{2}$ concentration by $690 \mathrm{ppm}$ will lead to $5-10 \%$ reductions in the concentration of iron, zinc, potassium, calcium, phosphorus, sulfur, magnesium, copper, and manganese in the majority of crops (6). The decline in zinc content due to the continuously increasing level of $\mathrm{CO}_{2}$ is expected to place 150 200 million people at risk of zinc deficiency (7). Mitigating the adverse effects of climate change on the nutritional quality of food crops can be achieved through breeding biofortified food crops.

Breeding biofortified crops require the identification of genetic resources with high micronutrient and mineral content from available germplasm. Most of the crops' wild relatives, landraces, and local cultivars are a rich source of these nutrients, which provide their effective utilization in the breeding program. The HarvestPlus biofortification program was started by International Food Policy Research Institute and International Center for Tropical Agriculture in collaboration with the Consultative Group on International Agricultural Research (CGIAR) centers which focused on making crops rich in vitamin $\mathrm{A}$, iron, and zinc. The target crops were beans and pearl millet for iron, maize, cassava, and sweet potato for vitamin A, and wheat, rice, and maize for zinc content. The sole aim of this project is to develop micronutrient-rich crops through breeding and delivery of these nutrient-rich crops in the developing world which are mainly affected by micronutrient malnutrition. The quality traits are polygenic and are quantitatively controlled; therefore, it is difficult to improve these traits by conventional breeding. The rapid development of advanced genomic tools like molecular markers provides an effective means for improving the efficiency of plant breeding in transferring these quantitatively inherited traits. The adoption of molecular markers can remarkably facilitate the breeding programs by identifying the exact location of the genomic region/QTL controlling the trait for nutrient content. The identified QTLs can then be easily transferred to elite breeding materials if these lines have a low level of nutrient content. The usefulness of these markers based on QTL mapping was not that significant because these are based on bi-parental mapping populations. However, genome-wide association mapping studies (GWAS) offer unique opportunities to use diverse germplasm which had accumulated a large number of meiotic events as opposed to only one or a few meiotic recombinations in bi-parental mapping populations. The ability to resolve marker/trait associations depends upon the extent of linkage disequilibrium present in the panel. The markers identified to be linked with QTL have the potential to be used across breeding material for identification and introgression. In this review, we aimed to compile information on QTLs pertaining to nutrient content in important food crops, which once biofortified, may ensure good nutrition and eliminate hunger among populations, especially food-insecure children who are majorly impacted by climate-related devastations.

\section{QTLs for Pro-vitamin A}

Vitamin A deficiency is one of the serious health problems in developing countries, leading to irreversible blindness. This situation encouraged the researchers to attempt the biofortification of plant-derived foods including pro-vitamin A carotenoids (mainly $\beta$-carotene), which led to "golden" crops. The conversion rates of $\beta$-carotene into vitamin $A$ are reported to be high in golden rice, maize, and cassava, demonstrating that these staple crops may incorporate a higher level of nutritional 


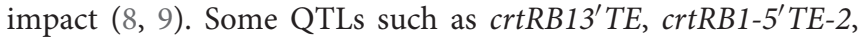
crtRB1-3'TE-1, crtRB1-5'TE-2, crtRB1-3'TE-1, LCYE, PSY1, and $c r t R b 1$ for pro-vitamin A have been identified on chromosome 10 in maize (10-13). In addition to this, QTL for $\beta$-carotene content has been reported in recombinant inbred line (RIL) populations through SSR markers in maize (14) (Table 1).

\section{QTLs for Fe and Zn Content}

Iron $(\mathrm{Fe})$ and zinc $(\mathrm{Zn})$ are among the essential micronutrients that are often lacking in human diet (4). According to WHO, over $30 \%$ of the population is anemic. These micronutrients play a wide range of metabolic functions required for normal growth and brain development. Zinc is an essential nutrient in the proper functioning of the immune system. Infants, small children, and pregnant and lactating women are at a higher risk of $\mathrm{Fe}$ and $\mathrm{Zn}$ depletion. Therefore, it is important to raise the level of $\mathrm{Fe}$ and $\mathrm{Zn}$ in the daily diet of children and women to save them from malnutrition. Cereals biofortified with $\mathrm{Fe}$ and $\mathrm{Zn}$ can eliminate the problem of malnutrition. These traits $(\mathrm{Zn}$ and $\mathrm{Fe}$ ) are polygenic in nature, and genetic variation for these traits exists in landraces and progenitor species of important food crops. Breeding strategies are therefore focusing on novel approaches to broaden the genetic base using wild/related species and landraces and identifying genetic control and their effects (107). Several genetic mapping populations have been developed in the past few years to dissect Fe- and Zn-related traits. In wheat, various studies have reported QTL for high grain $\mathrm{Fe}$ and $\mathrm{Zn}$ concentrations on chromosomes $1 \mathrm{~A}, 1 \mathrm{D}, 1 \mathrm{~B}, 2 \mathrm{~A}, 2 \mathrm{~B}, 3 \mathrm{~B}, 3 \mathrm{D}, 4 \mathrm{~B}$, $5 \mathrm{~A}, 6 \mathrm{~A}, 6 \mathrm{~B}, 7 \mathrm{~A}, 7 \mathrm{~B}$, and $7 \mathrm{D}$ in hexaploid wheat $(16,18,20-24)$ and on chromosome 2 in barley (25) (Table 1). Recently, Velu et al. (19) evaluated the HarvestPlus Association Mapping panel across a range of environments in India and Mexico. GWAS analysis revealed two larger QTL regions on chromosomes 2 and 7 for large grain $\mathrm{Zn}$ content.

In rice, QTLs ( $q F e$ 1.2, $q F e ~ 2$, and $q F e$ 6) were identified on chromosomes 1,2 , and 6 for Fe content $(37,38,41)$ and on chromosomes 5,8 , and $7(q Z n 5, q Z n$ 8) for $Z n$ content $(37,41,42)$. Most of the QTLs for Fe and Zn content were identified through single-nucleotide polymorphism (SNP) and DArT-SSR markers in RIL and BIL populations, but some of them were also from DH lines (Table 1). Recently, many new QTLs for Fe and Zn content have been mapped mainly through SSR markers in DH and BIL mapping populations $(26,27,29,34-36)$ (Table 1). Maize hybrids and varieties having a high yield potential along with $25-30 \%$ more $\mathrm{Fe}$ and $\mathrm{Zn}$ than common cultivars have been developed as part of the HarvestPlus program (108). Pearl millet is an important nutricereal rich in $\mathrm{Fe}$ and $\mathrm{Zn}$. Recently, Kumar et al. (46) and Anuradha et al. (47) have identified QTLs for grain Fe and $\mathrm{Zn}$ content in RILs and 130 pearl millet germplasm lines (Table 1).

Pulses have a uniformly higher amount of $\mathrm{Fe}$ and $\mathrm{Zn}$ compared to the cereals and are a better source of micronutrients. Izquierdo et al. (48), using a meta-QTL analysis in 7 populations, identified three major QTL regions governing seed Fe and Zn content and concentration in seeds. Many other QTLs for Fe and $\mathrm{Zn}$ content (Table 1) have also been reported in common bean using SSR markers $(49,51)$, in lentil using SNP markers $(54,55,58)$, and in peas using SSR (60) and SNP markers (59). In chickpea, Upadhyaya et al. (62) and Diapari et al. (64) identified QTLs for Fe and Zn content using SNP markers in germplasm lines (Table 1). Similarly in mungbean, two QTLs, viz., $q F e 4.1$ and $q Z n 6.4$ were mapped on chromosomes 4 and 6, respectively, using SSR markers in a RIL population (68). In soybean, Ning et al. (77) reported two QTLs, qZn11.1 and $q F e 3$, on chromosomes 11 and 3, respectively, for seed $\mathrm{Zn}$ and Fe content. In lentils, the diversity of genes relating to iron and zinc contents in seeds was studied using molecular markers, with implications for biofortification (109-111).

\section{QTLs for Oil Content}

Oil content is a classic but significant yield trait in groundnut, peanut, soybean, and various Brassica species. The fatty acid composition of various edible oil crops has various parameters such as nutrition, oxidative stability, and shelf-life that correlate with overall oil quality. So, modifying the oil content of the majority of vegetable oil crops is one of the breeding objectives, while breeding vital, healthy, and desired fatty acids should be envisaged for improving oil quality in the specific genotypes. Generally, a higher content of oleic acid and a lower content of linoleic acid (high oleic/linoleic acid ratio) in cooking/edible oil is reported to be healthier for human consumption. Many QTLs (Table 1) have been reported for oil content in diploid and amphiploid species of Brassica such as Brassica napus (87, 89-91, 94, 99) and Brassica carinata (102). In Brassica juncea, QTLs for glucosinolate, gluconapin, sinigrin, glucoiberin, gluconeobrasscin, gluconasturtin, and tocopherol content were mapped in RILs on chromosomes (J18, J1, J4, J9, J7, and J6, respectively) using SSR markers (103). Researchers have developed "canola-grade" mustard varieties containing low levels of erucic acid and glucosinolates. The erucic acid content in Brassicas is controlled by the FAE1 gene. CAPS markers for genes FAE1.1 and FAE1.2 were developed and successfully used in breeding programs in the improvement of $B$. juncea (104) and B. rapa (105), but when compared to simple PCRbased markers, CAPS markers are labor- and cost-intensive, which restrict their routine use by breeders. Recently, Saini et al. (112) assessed polymorphism in the upstream region of FAE1.1 and FAE1.2 genes, across the low erucic acid and high erucic acid genotypes, and developed PCR-based markers (FAE1.1P and FAE1.2P) based on this variability. These markers can be effectively used in marker-assisted selection for the development of low-erucic-acid varieties in B. juncea. In $B$. Oleracea, gene FAD3-2 for low $\alpha$-linolenic acid has been tagged by a gene-specific marker (106) (Table 1). Peanut and soybean are extensively cultivated in many parts of the world and are an important source of edible oil. Researchers are identifying QTLs for the development of peanut genotypes with good oil quality and desired fatty acid composition. Huang and co-workers (65) reported QTL qOCb3 for oil content in the $\mathrm{F}_{2}$ population, whereas Pandey and co-workers (67) reported a meta-QTL (mqOC) for oil content in the RIL population (Table 1). In soybean, seed oil content is under polygenic control and is sensitive to environmental effects. QTLs for oil content flanked 
by SSR marker $(76,78)$ and SNP markers $(83)$ have been reported in the RIL population.

\section{QTLs for Fatty Acid Content}

The trait associated with oil quality, measured in terms of its fatty acid composition, is an important agronomic trait that can eventually be tracked using molecular markers. Oils provide essential fatty acids along with an array of vitamins. Researchers have identified QTLs for major saturated (palmitic acid, arachidic acid, stearic acid, behenic acid, etc.) and unsaturated fatty acids (oleic acid, linoleic acid, linolenic acid, erucic acid, etc.) in major oilseed crops. The QTLs for major fatty acids will be discussed below.

Palmitic acid (C16:0) is the most common saturated fatty acid found in animals, plants, and microorganisms and is also the precursor to longer fatty acids (113). Many QTLs have been reported in major oilseed crops such as peanut $(65,66)$, soybean (83), and B. napus $(87,89,95,96,100)$ for this trait. The details of the marker linked to palmitic acid content are given in Table 1.

Stearic acid (C18:0) is a saturated fatty acid, and its ester is one of the most common saturated fatty acids found in nature following palmitic acid. Many QTLs controlling stearic acid content have been reported in several oil crops. In soybean, a major QTL (qSTEL001) has been reported using SNP markers on chromosome 16 in RIL population (83). In B. napus, a QTL (qC18:0-C3e) was reported on chromosomes $\mathrm{C} 8$ and $\mathrm{C} 3$ using DH lines (89). In peanuts, QTLs such as ASAA4, S_mqSAa07_1, and T_mqSA_b04_1 have been reported in $\mathrm{F}_{2}$ and RILs on chromosomes A4, A7, and A9 $(65,66)$.

Arachidic acid (C20:0) is a saturated fatty acid present in peanut seeds. Several QTLs have been identified in soybean, peanut, and Brassica species pertaining to it. In B. napus, QTL $\mathrm{qC} 18: 3-\mathrm{C} 3 \mathrm{c}$ was present on chromosomes $\mathrm{C} 3$ and $\mathrm{C} 8$ in $\mathrm{DH}$ lines (87). QTLs such as $q A A B 9, S \_m q A A \_b 098$, and T_mqAA_b04_1 in peanut were identified on chromosome B9 using F2 and RILs population $(65,66)$. The details of the marker linked to arachidic acid content are given in Table $\mathbf{1}$.

Oleic acid (C18:1) is a naturally occurring fatty acid among various animal and vegetable oils. Oleic acid accounts for about $80 \%$ of peanut oil. Norden et al. (114) have identified two high oleic acid peanut lines (F435-2-1 and F435-2-2) through a systematic breeding program in Florida. In the same year, genetics of this trait came before the world. Moore and Knauft (115) identified two homozygous recessive mutant genes FAD2A and FAD2B for high oleic acid content, but this was at the cost of lower linoleic acid. As time progressed, many reports have come out on mapping of this trait. In peanut, QTLs for high oleic acid content have been reported by many researchers (Table $\mathbf{1}$ ). Through the DArTseq marker, a QTL (OLE-WH14) was reported on chromosome B2 in inbred lines and another QTL (qOLE.C41) was also reported on chromosome $\mathrm{C} 4$ in $\mathrm{DH}$ lines in $B$. carinata (102). Similarly, in B. Napus, QTLs (BnaA05g 23520, BnaA05g 23930, Gene BrFAD5, and Gene BrFAD7) were detected for oleic acid using DH lines as mapping populations (Table $\mathbf{1}$ ). In soybean, a QTL (qOLE003) was reported using SNPs in the RIL population. In peanut, a QTL ( $q O A A 3)$ was identified in $\mathrm{F}_{2}$ and another QTL ( $m q$ QA181) in RILs population on chromosomes $\mathrm{A} 3$ and $\mathrm{B} 9$ (Table 1).

Linoleic acid (C18:2) is about $40 \%$ in normal peanut lines. Pandey and co-workers (67) reported the relationship of FAD2 genes with peanut oil quality and suggested that FAD2B contributed higher phenotypic variance for linoleic acid than the FAD2A alleles. Recently, $\mathrm{Hu}$ et al. (116) detected the two main QTLs for linoleic acid content located in linkage groups A09 and B09 in peanut. They reported that SNP markers, viz., Marker2575339 and Marker2379598 in B09, were associated with oleic acid and linoleic acid in seven environments and Marker4391589 and Marker-4463600 in A09 in six environments. The QTLs for linoleic acid were identified in other crops, too, viz., soybean (qLINN007), B. carinata (LEN-WH14, qLEI.B8-4), and B. napus (uqFA-C3-3, uqFA-A8-4, qC182_N9, FAD7, FAD3A, and FAD $3 C$ genes) using RILs, DHs, and inbred lines (Table 1).

\section{QTLs for Protein and Amino Acid Content}

Pulses are important sources of proteins, important nutrients, and calories, particularly in diets of peoples living in developing countries. Paying less attention to the genetic enhancement of pulses is likely to have a noticeable impact on global food and nutritional security (117). Advances in sequencing technologies have made significant improvements in the breeding lines of important pulses such as pigeonpea, chickpea, and groundnut; however, the pace in improvement does not match that of cereals. Importantly, the consumption of pulses has been shown to have several positive health effects that are inherently tied to their nutritional qualities (118). The development of improved cultivars of pulses can be accelerated through the identification and the deployment of the gene(s)/QTLs of nutritional quality traits. In chickpea germplasm lines (Desi and Kabuli), two significant QTLs for protein content linked with SSR markers TR26.205 and CaM1068.195, present on LG3 and LG5, were reported (63). In soybean, major QTLs (qProt_Gm20, $q P R O \_13 \_1, q P R O 001$, and $\left.q P C\right)$ for protein content flanked by SNP and SSR markers were identified in RILs $(66,73,79,83)$. In B. carinata, QTLs for protein content were identified ( $q P R O 10$ and PRO-WH13) through DArTseq marker using DH and inbred lines (102) (Table 1). Among cereals, rice and wheat are also sources of protein in daily diets, though in limited quantity. The major effect of QTLs for grain protein content has been mapped in rice $(28,33,39,43)$ and Wheat $(15,17,22)$ (Table 1).

Protein is composed of amino acids; these amino acids are the "building blocks" of the body, and improving essential amino acids is one of the better prospects envisaged. Some QTLs corresponding to essential amino acids have been identified in important food crops. Amino acid contents such as those of lysine, threonine, methionine, and cysteine were improved in soybean through the identification of QTLs in different RIL populations $(70,71,73,80)$. In B. napus, QTLs were identified for lysine, threonine, and methionine content (qLysC-16-3, qThrC12-5, and qMetC-9-5) in DH lines on chromosomes C6, C2, and A9 (93). In B. napus, arginine, histidine, glutamic acid, glycine, alanine, and aspartic acid content was improved through QTLs such as $q$ ArgC-8-5, qHisC-4-3, qGluC-1-1, qGlyC-4-1, qProC-1-1, $q A l a C-7-3$, and $q A s p C-9-4$ in $\mathrm{BC}$ lines on chromosomes A1, A4, 
TABLE 1 | List of important QTLs pertaining to nutritional quality in major food crops.

\begin{tabular}{|c|c|c|c|c|c|c|c|}
\hline Crop & QTL /Loci & MP & Cross(s) & Markers & Chromosome & $\begin{array}{l}\text { Nutritional parameters } \\
\text { in the grain }\end{array}$ & References \\
\hline \multirow[t]{10}{*}{ Wheat } & $\begin{array}{l}\text { QGpc-1B-2 } \\
\text { QGpc-4B-1.4 }\end{array}$ & RILS & Tainong 18/Linmai 6 & $\begin{array}{l}\text { D-1190331S-3222160 (SNP) } \\
\text { D-1380792D-1094306(SNP) }\end{array}$ & $\begin{array}{l}1 \mathrm{~B} \\
4 \mathrm{~B}\end{array}$ & Grain protein content & (15) \\
\hline & $\begin{array}{l}\text { QGZn.co-5A } \\
\text { QGZn.co-7A } \\
\text { QGFe.co-3B.1 } \\
\text { QGFe.co-5A.2 }\end{array}$ & RILs & Roelfs F2007/Hong Hua Mai/./Blouk \#1 & $\begin{array}{l}\text { 1244217-1272027|F|O (DArT) } \\
5356706-5325178|\mathrm{~F}| \mathrm{O} \text { (DArT) } \\
\text { 1089107-1127875|F|O (DArT) } \\
1102433-988523 \text { (DArT) }\end{array}$ & $\begin{array}{l}5 A \\
7 A \\
3 B \\
5 A\end{array}$ & $\begin{array}{l}\mathrm{Zn} \\
\mathrm{Fe}\end{array}$ & (16) \\
\hline & $\begin{array}{l}\text { QGpc.uhw-4B } \\
\text { QGpc.uhw-5A.1 } \\
\text { QGpc.uhw-6B } \\
\text { QGpc.uhw-7B.2 }\end{array}$ & RILs & Svevo/Y12-3 & $\begin{array}{l}\text { TG0010b(SNP) } \\
\text { RAC875_rep_c106118-339(SNP) } \\
\text { T.durum contig 9860-281(SNP) } \\
\text { Kukri_c14766-484(SNP) }\end{array}$ & $\begin{array}{l}4 \mathrm{~B} \\
5 \mathrm{~A} \\
6 \mathrm{~B} \\
7 \mathrm{~B}\end{array}$ & Grain protein content & $(17)$ \\
\hline & $\begin{array}{l}\text { qFes1 } \\
\text { qfes2 } \\
\text { qZns1 } \\
\text { qZns2 }\end{array}$ & Inbreds & Synthetic hexaploid wheat & $\begin{array}{l}\text { Xwmc399 } \\
\text { Xgwm157 } \\
\text { Xwmc357 } \\
\text { Xcfd63 }\end{array}$ & $\begin{array}{l}4 D \\
2 D \\
5 D \\
1 D\end{array}$ & $\begin{array}{l}\mathrm{Fe} \\
\mathrm{Zn}\end{array}$ & (18) \\
\hline & $\begin{array}{l}\text { QZn 2A } \\
\text { QZn7B }\end{array}$ & Inbreds & HPAM panel genotypes & $\begin{array}{l}\text { RAC875_c34757_180 (SNP) } \\
\text { wsnp_Ex_c5268_9320618 (SNP) }\end{array}$ & $\begin{array}{l}2 \mathrm{~A} \\
7 \mathrm{~B}\end{array}$ & $\begin{array}{l}\mathrm{Zn} \\
\mathrm{Zn}\end{array}$ & (19) \\
\hline & $\begin{array}{l}\text { QGzn.ada_1B } \\
\text { QGzn.sar_1B } \\
\text { QGFe.ada_2B }\end{array}$ & RILs & $\begin{array}{l}\text { Adana/70711 Saricanak 98/MM S4 } \\
\text { Adana/70711 }\end{array}$ & $\begin{array}{l}\text { rpt-6561 (DArT) } \\
\text { wPt- } 6434 \text {-wPt1403 (DArT) } \\
\text { wPt-9812 (DArT) }\end{array}$ & $\begin{array}{l}1 \mathrm{~B} \\
1 \mathrm{~B} \\
2 \mathrm{~B}\end{array}$ & $\begin{array}{l}\mathrm{Zn} \\
\mathrm{Zn} \\
\mathrm{Fe}\end{array}$ & (20) \\
\hline & $\begin{array}{l}\text { QZn.Across_4BS } \\
\text { QFe.Across_7DS }\end{array}$ & RILs & Seri M 82/SHW Cw176364 & $\begin{array}{l}\text { TP91631-TP81797 (SNP) } \\
\text { TP43715-TP37547 (SNP) }\end{array}$ & $\begin{array}{l}4 \mathrm{BS} \\
7 \mathrm{DS}\end{array}$ & $\begin{array}{l}\mathrm{Zn} \\
\mathrm{Fe}\end{array}$ & (21) \\
\hline & $\begin{array}{l}\text { QZn.bhu_2B, QFe.bhu_2B } \\
\text { QGPC.bhu_1A }\end{array}$ & $\mathrm{DHs}$ & Berkut/Krichaff & $\begin{array}{l}\text { gwm120-wPt2430 (SSR-DArT) } \\
\text { wPt 9592-GBM } 1153 \text { (DArT-SSR) }\end{array}$ & $\begin{array}{l}2 B \\
1 A\end{array}$ & $\begin{array}{l}\text { Zn and } \\
\mathrm{Fe} \\
\text { Protein content }\end{array}$ & (22) \\
\hline & $\begin{array}{l}\text { QZn.bhu_2B } \\
\text { QZn.bhu_6A } \\
\text { QFe.bhu_3B }\end{array}$ & RILs & PI 348449 (T. spelta)/HUW 234 & $\begin{array}{l}\text { 989092F0 (SNP) } \\
\text { 998265F0 (DArT) } \\
\text { 3022954FO (SNP) }\end{array}$ & $\begin{array}{l}2 B \\
6 A \\
3 B\end{array}$ & $\begin{array}{l}\mathrm{Zn} \\
\mathrm{Zn} \\
\mathrm{Fe}\end{array}$ & (23) \\
\hline & QGzncpk.cimmyt_2BL & RILs & PBW 343/Kenya Swara & wPt-6174 (DArT) & $2 \mathrm{BL}$ & $\mathrm{Zn}$ & (24) \\
\hline Barley & QTL.Zn & RILs & Clipper/Sahara & $\begin{array}{l}\text { Xbcd175-Xpsr108 } \\
\text { Vrs1-XksuF15 }\end{array}$ & $\begin{array}{l}2 \mathrm{HS} \\
2 \mathrm{HL}\end{array}$ & $\mathrm{Zn}$ & (25) \\
\hline \multirow[t]{3}{*}{ Rice } & $\begin{array}{l}\text { QTL.Fe.9 } \\
\text { QTL.Zn.4 } \\
\text { QTL.pro.1 }\end{array}$ & Inbreds & Landraces & $\begin{array}{l}\text { RM215 (SSR) } \\
\text { RM551 (SSR) } \\
\text { RM5 (SSR) }\end{array}$ & $\begin{array}{l}9 \\
4 \\
1\end{array}$ & $\begin{array}{l}\mathrm{Fe} \\
\mathrm{Zn} \\
\text { Protein content }\end{array}$ & (26) \\
\hline & $\begin{array}{l}q F e 3.3 \\
q F e 7.3 \\
q Z n 2.2 \\
q Z n 8.3\end{array}$ & Inbreds & Germplasm lines & $\begin{array}{l}\text { RM7 (SSR) } \\
\text { RM1132 (SSR) } \\
\text { RM300 (SSR) } \\
\text { RM80 (SSR) }\end{array}$ & $\begin{array}{l}3 \\
7 \\
2 \\
8\end{array}$ & $\begin{array}{l}\mathrm{Fe} \\
\mathrm{Zn}\end{array}$ & $(27)$ \\
\hline & $\begin{array}{l}\text { qAAC6.1 } \\
\text { qAAC7.1 } \\
q P C 1.2\end{array}$ & RILs & Milyang23/Tong88-7 & $\begin{array}{l}3515361-3658340(\text { SNP) } \\
4856196-5206110(\text { SNP) } \\
39162572-39234399 \text { (SNP) }\end{array}$ & $\begin{array}{l}6 \\
7 \\
1\end{array}$ & $\begin{array}{l}\text { Amino acid content } \\
\text { Protein content }\end{array}$ & (28) \\
\hline
\end{tabular}




\begin{tabular}{|c|c|c|c|c|c|c|c|}
\hline Crop & QTL /Loci & MP & Cross(s) & Markers & Chromosome & $\begin{array}{l}\text { Nutritional parameters } \\
\text { in the grain }\end{array}$ & References \\
\hline & $\begin{array}{l}\text { qFe1.1 } \\
\text { qFe1.2, qZn1.1 } \\
\text { qFe6.1, qZn6.1 } \\
\text { qFe6.2, qZn6.2 }\end{array}$ & BILs & RP-Bio226/Sampada & $\begin{array}{l}\text { RM562-RM11943 (SSR) } \\
\text { RM294A-RM12276 (SSR) } \\
\text { RM8226-RM400 (SSR) } \\
\text { RM400-RM162 (SSR) }\end{array}$ & $\begin{array}{l}1 \\
1 \\
6 \\
6\end{array}$ & $\begin{array}{l}\mathrm{Fe} \\
\mathrm{Fe} \text { and } Z \mathrm{n} \\
\mathrm{Fe} \text { and } Z \mathrm{n} \\
\mathrm{Fe} \text { and } Z \mathrm{n}\end{array}$ & (29) \\
\hline & $\begin{array}{l}q A A 1 \\
q A A 7\end{array}$ & RILs & Zhenshan 97/Delong 208 & $\begin{array}{l}\text { RM493-RM562 } \\
\text { MRG186-MRG4499 }\end{array}$ & $\begin{array}{l}1 \\
7\end{array}$ & Amino acid content & (30) \\
\hline & qPC6.2 & RILs & Yukihikari/Joiku 462 & Indel 207-Indel 208 & 6 & Protein content & (31) \\
\hline & $\begin{array}{l}\text { qB4.1 } \\
\text { qCo7.1 } \\
\text { qCu8.1 } \\
\text { qK6.1 } \\
\text { qMn3.1 } \\
\text { qMg1.1 } \\
\text { qZn11.1 } \\
\text { qP11.2 }\end{array}$ & $\mathrm{DH}$ & $\begin{array}{l}\text { R64/IR69428 (Pop1) and BR29/IR75862 } \\
\text { (Pop2) }\end{array}$ & $\begin{array}{l}\text { 4333768-4349423(SNP) } \\
\text { 7872824-7892971(SNP) } \\
\text { 89266819-8940497(SNP) } \\
5883472-5895767(\text { SNP) } \\
3005168-2732340(\text { SNP) } \\
115078-117345(\text { SNP) } \\
10907196-11001107(\text { SNP) } \\
11391672-11407547(\text { SNP) }\end{array}$ & $\begin{array}{l}4 \\
7 \\
8 \\
6 \\
3 \\
1 \\
11 \\
11\end{array}$ & $\begin{array}{l}\text { Boron } \\
\text { Cobalt } \\
\text { Copper } \\
\text { Potassium } \\
\text { Manganese } \\
\text { Magnesium } \\
\text { Zinc } \\
\text { Phosphorous }\end{array}$ & (32) \\
\hline & $\begin{array}{l}\text { qGPC1.1 } \\
\text { qSGPC2.1 } \\
\text { qSGPC7.1 }\end{array}$ & BILs & ARC10075/Naveen & $\begin{array}{l}93237905-93229368(\mathrm{SNP}) \\
93256429-93260438(\mathrm{SNP}) \\
93225742-93256949(\mathrm{SNP})\end{array}$ & $\begin{array}{l}1 \\
2 \\
7\end{array}$ & Grain protein content & (33) \\
\hline & $\begin{array}{l}q F e 7 \\
q Z n 7\end{array}$ & $\mathrm{DH}$ & Goami2/Hwaseonchal & $\begin{array}{l}\text { RM10-RM1973 (SSR) } \\
\text { RM10-RM1973 (SSR) }\end{array}$ & 7 & $\begin{array}{l}\mathrm{Fe} \\
\mathrm{Zn}\end{array}$ & (34) \\
\hline & $\begin{array}{l}\text { qFe1.2 } \\
\text { qFe11.1 } \\
\text { qZn2.1 } \\
\text { qZn3.2 } \\
\text { qFe3.2 } \\
\text { qFe4.1 } \\
\text { qZn5.1 } \\
\text { qZn12.1 }\end{array}$ & BILs & $\begin{array}{l}\text { Swarna/O. nivara (IRGC81832) P1 Swarna/O. } \\
\text { nivara (IRGC81848) P2 }\end{array}$ & $\begin{array}{l}\text { RM243-RM81A (SSR) } \\
\text { RM332-RM287 (SSR) } \\
\text { RM250-RM535 (SSR) } \\
\text { RM55-RM520 (SSR) } \\
\text { RM520-RM514 (SSR) } \\
\text { RM241-RM348 (SSR) } \\
\text { RM153-RM413 (SSR) } \\
\text { RM415-RM19 (SSR) }\end{array}$ & $\begin{array}{l}1 \\
11 \\
2 \\
3 \\
3 \\
4 \\
5 \\
12\end{array}$ & $\begin{array}{l}\mathrm{Fe} \\
\mathrm{Fe} \\
\mathrm{Zn} \\
\mathrm{Zn} \\
\mathrm{Fe} \\
\mathrm{Fe} \\
\mathrm{Zn} \\
\mathrm{Zn}\end{array}$ & (35) \\
\hline & $\begin{array}{l}\text { qFe10.1 } \\
\text { qZn6.2 } \\
\text { qZn7.1 }\end{array}$ & MAGIC & MAGIC Population & $\begin{array}{l}\text { S10_13883426 } \\
\text { S6_2939487 } \\
\text { S6_29350591 }\end{array}$ & $\begin{array}{l}10 \\
6 \\
7\end{array}$ & $\begin{array}{l}\mathrm{Fe} \\
\mathrm{Zn} \\
\mathrm{Zn}\end{array}$ & (36) \\
\hline & $\begin{array}{l}q F e \quad 6 \text { and } \\
q Z n 8\end{array}$ & BILs & IR 7586/Ce 258 IR 7586/Zhongguangxiang1 & $\begin{array}{l}\text { RM3- RM340 (SSR) } \\
\text { RM407- RM152 (SSR) }\end{array}$ & $\begin{array}{l}6 \\
8\end{array}$ & $\begin{array}{l}\mathrm{Fe} \\
\mathrm{Zn}\end{array}$ & (37) \\
\hline & $\begin{array}{l}\text { qFe } 1.2 \text { (gene OsYSL1) } \\
\text { qFe } 5.1 \text { (gene OsZIP6) } \\
\text { qFe } 7.2 \text { (gene OsZIP8) }\end{array}$ & RILs & Madhukar/Swarna & $\begin{array}{l}\text { RM490 (SSR) } \\
\text { RM574 (SSR) } \\
\text { RM8007 (SSR) }\end{array}$ & $\begin{array}{l}1 \\
5 \\
7\end{array}$ & $\mathrm{Fe}$ & (38) \\
\hline & $\begin{array}{l}\text { qPro-2 } \\
\text { qPro-10 } \\
\text { qLip-6 }\end{array}$ & $\mathrm{DHs}$ & Cheongcheong/Nagdong & $\begin{array}{l}\text { RM5619-RM1211 (SSR) } \\
\text { RM24934-RM25128 (SSR) } \\
\text { RM586-RM1163 (SSR) }\end{array}$ & $\begin{array}{l}2 \\
10 \\
6\end{array}$ & $\begin{array}{l}\text { Protein content C } \\
\text { Protein content LC }\end{array}$ & (39) \\
\hline & qPro-2 & $\mathrm{DHs}$ & Cheongcheong/Nagdong & RM12532-RM555 & 2 & $\mathrm{PC}$ & (40) \\
\hline & $\begin{array}{l}\text { qFe 2, qZn 5, qCo 1, qCu 2, qMn } \\
\text { 7, and qMo } 7\end{array}$ & BILs & Teqing/Lemont & $\begin{array}{l}\text { RM452, RM421, RM490, RM6378, RM214, } \\
\text { and RM11 (SSR) }\end{array}$ & \multicolumn{2}{|c|}{ 2, 5, 1, 2, 7and 7Fe, Zn, Co, Cu, Mn, Mo } & (41) \\
\hline
\end{tabular}


TABLE 1 | Continued

\begin{tabular}{|c|c|c|c|c|c|c|c|}
\hline Crop & QTL /Loci & MP & Cross(s) & Markers & Chromosome & $\begin{array}{l}\text { Nutritional parameters } \\
\text { in the grain }\end{array}$ & References \\
\hline & $\begin{array}{l}\text { OsZIP8a } \\
\text { OsNAC } \\
\text { OsZIP4b }\end{array}$ & RILS & IRRI 38/Jeerigesanna & $\begin{array}{l}\text { RM8007 (SSR) } \\
- \\
-\end{array}$ & $\begin{array}{l}7 \\
3 \\
8\end{array}$ & $\mathrm{Zn}$ & (42) \\
\hline & $q P C 1$ & RILS & Zhenshan97/Nanyangzhan & RM472- RM104 & 1 & Protein content & (43) \\
\hline \multirow[t]{7}{*}{ Maize } & crtRB13'TE & Inbred & $\begin{array}{l}\text { 1.V335/HP465-30 } \\
\text { 2.V345/HP465-35 }\end{array}$ & crtRB1-3’TE-1 (SSR) & 10 & Pro-vitamin A & (12) \\
\hline & $\begin{array}{l}\text { crtRB1-5'TE-2 } \\
\text { crtRB1-3'TE-1 }\end{array}$ & Inbreds & Нp321-1 & $\begin{array}{l}\text { crtRB1-5'TE-2 (SSR) } \\
\text { crtRB1-3'TE-1 (SSR) }\end{array}$ & 10 & Pro-vitamin A & (13) \\
\hline & $\begin{array}{l}\text { LCyE } \\
\text { CrtRB1-3'TE }\end{array}$ & $\begin{array}{l}F_{2} \\
F_{3: 4}\end{array}$ & $\begin{array}{l}\mathrm{L} 1-\mathrm{L} 5 \\
\mathrm{H} 1-\mathrm{H} 15\end{array}$ & $\begin{array}{l}\text { LcyE-5'TE (SSR) } \\
\text { LcyE-3'TE (SSR) } \\
\text { crtRB1-3'TE (SSR) }\end{array}$ & 10 & Pro-vitamin A & (10) \\
\hline & $\begin{array}{l}\text { LCYE } \\
\text { PSY1 } \\
\text { CrtRb1 }\end{array}$ & Inbreds & 130 Tropical Inbred lines & $\begin{array}{l}\text { LCYE-5'TE (285 Indel) } \\
\text { LCYE-SNP (G-C SNP) } \\
\text { LCYE-3'TE (8 bp Indel) } \\
\text { PSY-SNP7 (A-C SNP) } \\
\text { PSY1-1D1 ( } 378 \text { Indel) } \\
\text { crtRB1-5'TE (397/206 bp Indel) } \\
\text { crtRB1-InDel4 (12 bp Indel) } \\
\text { crtRB1-3'TE (325/1250 bp Indel) }\end{array}$ & & Pro-vitamin A & (11) \\
\hline & crtRB1 & RILs & $\begin{array}{l}\text { 1.B73/BY804 } \\
\text { 2.A619/SC55 } \\
\text { 3.K13/B77 } \\
\text { 4.K13/SC55 }\end{array}$ & $\begin{array}{l}\text { umc1506-bning1028 } \\
\text { umc1506-crtRB1 } \\
\text { crtRB1 } \\
\text { crtRB1 }\end{array}$ & 10 & $\begin{array}{l}\beta \text {-carotene, } \beta \mathrm{C} / \beta \mathrm{CX}, \beta \mathrm{C} / \mathrm{Z} \\
\text { and } \beta \mathrm{C} / \mathrm{ALL}\end{array}$ & (14) \\
\hline & $\begin{array}{l}\text { sQTL4.2 } \\
\text { sQTL4.1 } \\
\text { sQT/3.1 }\end{array}$ & RILS & Ye478/Wu312 & $\begin{array}{l}\text { umc1620-umc1994 (SSR) } \\
\text { umc1346-bnlg2291 (SSR) } \\
\text { mmc0132-umc1504 (SSR) }\end{array}$ & $\begin{array}{l}4 \\
4 \\
3\end{array}$ & $\begin{array}{l}\mathrm{Zn} \\
\mathrm{Mn} \\
\mathrm{Mg}\end{array}$ & (44) \\
\hline & $\begin{array}{l}\text { qZn5, qMn1, qCo3, qCu8, qK4, } \\
\text { qMo1, qNa5, qP4, qS1 }\end{array}$ & RILS & B73/LL14H & $\begin{array}{l}\text { PZA02411.3, PZA02135.2, PZA01925.1, } \\
\text { PZA03698.1, PZA01751.1, PZA02269.3.4, } \\
\text { PZA01327.1, PZA01751.2, PZA02698.3 } \\
\text { (All SNP) }\end{array}$ & $\begin{array}{l}5,1,3,5,8,4 \\
1,5,4,1\end{array}$ & $\begin{array}{l}\mathrm{Zn}, \mathrm{Mn}, \mathrm{Co}, \mathrm{Cu}, \mathrm{K}, \mathrm{Mo} \\
\mathrm{Na}, \mathrm{P}, \mathrm{S}\end{array}$ & (45) \\
\hline \multicolumn{2}{|c|}{ Pearl millet $q F e 1 / 54$ and $q Z n 1 / 54$} & RIL & $\begin{array}{l}\text { ICMS 8511-S1-17-2-1-1-B-P03_AIMP } \\
\text { 92901-S1-183-2-2-B-08 }\end{array}$ & Pgpb10531-pgpb9130 (DArT) & LG1 & Fe and $\mathrm{Zn}$ & (46) \\
\hline & $F e$ and $Z n$ & Inbreds & 130 germplasm lines & Xpsmp2261, Xipes0180, Xipes0096 & & Fe and $Z n$ & (47) \\
\hline \multirow[t]{4}{*}{$\begin{array}{l}\text { Common } \\
\text { Bean }\end{array}$} & $\begin{array}{l}\text { MQTL_Fe,Zn } 1.1 \\
\text { MQTL_Fe,Zn } 6.1 \\
\text { MQTL_Fe,Zn } 6.1\end{array}$ & RILS & 7 Populations & & $\begin{array}{l}1 \\
6 \\
6\end{array}$ & $F e$ and $Z n$ & (48) \\
\hline & $\begin{array}{l}\text { Seed coat } F e \\
\text { Seed coat } Z n\end{array}$ & BILS & Cerinza/G10022 & $\begin{array}{l}\text { Pv-at03, ATA16, ATA26 } \\
\text { ATA247, ATA10 }\end{array}$ & $\begin{array}{l}4,2,3 \\
8,6\end{array}$ & $\begin{array}{l}\text { Seed coat Fe } \\
\text { Seed coat } Z n\end{array}$ & (49) \\
\hline & $\begin{array}{l}\text { Ca1, Ca7, Ca9 } \\
\text { Mg7 } \\
\text { Pt5, Pt7 } \\
\text { DF6, DF7 }\end{array}$ & RILs & Xana/Cornell 49242 & $\begin{array}{l}\text { McatEtc, P gene, McagEac } \\
\text { P gene } \\
\text { OD12 } \\
\text { DBD }(A C), \text { SAS gene }\end{array}$ & $\begin{array}{l}1,7,9 \\
7 \\
5,7 \\
6,7\end{array}$ & $\begin{array}{l}\mathrm{Ca} \\
\mathrm{Mg} \\
\text { Protein content } \\
\text { Dietary fiber }\end{array}$ & (50) \\
\hline & $\begin{array}{l}\text { Fe_cont8.1 } \\
\text { Zn_cont2.1, 5.1, } 5.2 \text { and } 7.1\end{array}$ & BILs & Cerinza/G10022 & $\begin{array}{l}\text { BMc316 (SSR) } \\
\text { PV109, BM155, BMd28 and PV35 (SSR) }\end{array}$ & $\begin{array}{l}8 \\
3,5,5,7\end{array}$ & $\begin{array}{l}\mathrm{Fe} \\
\mathrm{Zn}\end{array}$ & (51) \\
\hline
\end{tabular}




\begin{tabular}{|c|c|c|c|c|c|c|c|}
\hline Crop & QTL /Loci & MP & Cross(s) & Markers & Chromosome & $\begin{array}{l}\text { Nutritional parameters } \\
\text { in the grain }\end{array}$ & References \\
\hline & $\begin{array}{l}F e-A A S 2 a \\
F e-A A S 6 c \\
Z n-A A S 2 c \\
Z n-A A S 7 c\end{array}$ & RILs & G21242/G21078 & $\begin{array}{l}\text { E0403A (SSR) } \\
\text { N0401B (SSR) } \\
\text { Pv11 (SSR) } \\
\text { BM239 (SSR) }\end{array}$ & $\begin{array}{l}2 \\
6 \\
2 \\
7\end{array}$ & $\begin{array}{l}\text { Fe concentration } \\
\text { Zn concentration }\end{array}$ & (52) \\
\hline & $\begin{array}{l}\text { MnQTL1.1 } \\
\text { MnQTL3.2 } \\
\text { MnQTL3.3 }\end{array}$ & RILs & CDC Redberry/ILL7502 & SNP & $\begin{array}{l}\text { LG } \\
\text { LG3 } \\
\text { LG3 }\end{array}$ & Mn content & (53) \\
\hline \multirow[t]{5}{*}{ Lentil } & $\begin{array}{l}\text { FeQTL1.2, 2.2, 4.2, 5.2, 6.2, } \\
\text { and } 7.1\end{array}$ & RILs & ILL8006/CDC Milestone & $\begin{array}{l}\text { 15SNP, } 81 \text { SNP, } 40 \text { SNP, } 239 \text { SNP, } 4 \text { SNP and } \\
12 \text { SNP }\end{array}$ & $1,2,4,5,6,7$ & Fe concentration & (54) \\
\hline & Zn content & Inbreds & 143 diverse germplasm lines & $\begin{array}{l}\text { LcC06739p564(SNP) } \\
\text { LcC04105p1090 (SNP) }\end{array}$ & $\begin{array}{l}3 \\
2\end{array}$ & Zn content & (55) \\
\hline & SeQTL2.1, 5.1, 5.2, 5.3, & RILs & PI320937/Eston & SNP & $2,5,5,5$ & Selenium content & (56) \\
\hline & qFe uptake & RILs & ILL8006-BM/CDC Milestone & - & - & Fe uptake & (57) \\
\hline & $\begin{array}{l}121 \text { QTLs for Mn and } \\
\text { Zn concentartion }\end{array}$ & RILs & CDC-Redberry/ILL7602 & - & - & Mn and $Z n$ concentartion & (58) \\
\hline \multirow[t]{3}{*}{ Pea } & $\begin{array}{l}{[B]-P s 5.1,[\mathrm{Ca}]-P s 5.1,[M g]-P s 5.1} \\
{[S]-P s 5.1} \\
{[\text { Fe]-Ps7.1, }[\mathrm{Zn}]-P s 7.1} \\
{[K]-P s 5.1} \\
{[M n]-P s 5.1} \\
{[M o]-P s 5.1} \\
{[P]-P s 3.1}\end{array}$ & RILs & Aragorn/Kiflica & $\begin{array}{l}\text { TP61763 (SSR) } \\
\text { TP44143 (SSR) } \\
\text { TP55189 (SSR) } \\
\text { tip_SNP2_V (SNP) } \\
\text { TP42330 (SSR) } \\
\text { TP75231 (SSR) }\end{array}$ & $\begin{array}{l}5 \\
7 \\
5 \\
5 \\
5 \\
3\end{array}$ & $\begin{array}{l}\mathrm{B}, \mathrm{Ca}, \mathrm{Mg} \mathrm{S} \\
\mathrm{Fe}, \mathrm{Zn} \\
\mathrm{K} \\
\mathrm{Mn} \\
\mathrm{Mo} \\
\mathrm{P}\end{array}$ & (59) \\
\hline & $\begin{array}{l}\text { QTL.Fe1 } \\
\text { QTL.Fe2 } \\
\text { QTL.Fe3 }\end{array}$ & RILs & Carrera/CDC Striker & $\begin{array}{l}\text { Sc1203_101100 and PsC17710p220 } \\
\text { Sc9618_162688 and PsC4833p179 } \\
\text { Sc2559_48386 and PsC908p622 } \\
\text { (All SNP) }\end{array}$ & $\begin{array}{l}3 \\
4 \\
7\end{array}$ & Fe concentration & (60) \\
\hline & QTL FEBIO & RILs & 1-2347-144/CDC Meadow & PA-P (SNP) & 5 & Fe bioavailability & (61) \\
\hline Chickpea & $\begin{array}{l}\text { CaqFe1.1 } \\
\text { CaqZn2.1 } \\
\text { CaqFZ4.1 } \\
\text { CaqFZ5.1 } \\
\text { CaqFZ7.1 }\end{array}$ & Inbreds & 92 Germplasm lines & $\begin{array}{l}\text { SNP53-SNP55 } \\
\text { SNP110 } \\
\text { SNP300 } \\
\text { SNP413 } \\
\text { SNP471-SNP472 }\end{array}$ & $\begin{array}{l}1 \\
2 \\
4 \\
5 \\
7\end{array}$ & $\begin{array}{l}\mathrm{Fe} \\
\mathrm{Zn} \\
\mathrm{Fe} \text { and } \mathrm{Zn} \\
\mathrm{Fe} \text { and } \mathrm{Zn} \\
\mathrm{Fe} \text { and } \mathrm{Zn}\end{array}$ & (62) \\
\hline & QTL.PC & Inbreds & 187 Germplasm lines & TR26.205 (SNP), CaM1068.195 (SNP) & 3,5 & Protein content & (63) \\
\hline & $\begin{array}{l}\text { QTL.Zn } \\
\text { QTL.Fe }\end{array}$ & Inbreds & 94 Germplasm lines & $\begin{array}{l}\text { Cav1Sc25.1p2052607 } \\
\text { Cav1Sc19.1p1556596 }\end{array}$ & $\begin{array}{l}4 \\
1\end{array}$ & $\begin{array}{l}\mathrm{Zn} \\
\mathrm{Fe}\end{array}$ & (64) \\
\hline Peanut & $\begin{array}{l}\text { qOCB3 } \\
\text { qPAA8 } \\
\text { qOAA3 } \\
\text { qEAA5 } \\
\text { qBAB9 } \\
\text { qAAB9 } \\
\text { ASAA4 }\end{array}$ & $\mathrm{F}_{2}$ & Zhonghua10/ICG12625 & $\begin{array}{l}\text { AHGS1788-pPGSseq14C11 } \\
\text { PM54-pPGPSseq2G3 } \\
\text { TC4E10-ARS744 } \\
\text { GM1577-TC6E1 } \\
\text { AHGS1969 } \\
\text { AHGS1969 } \\
\text { GM2480 }\end{array}$ & $\begin{array}{l}\text { B3 } \\
\text { A8 } \\
\text { A3 } \\
\text { A5 } \\
\text { B9 } \\
\text { B9 } \\
\text { A4 }\end{array}$ & $\begin{array}{l}\text { Oil content } \\
\text { Palmatic acid } \\
\text { Oleic acid } \\
\text { Eicosenic acid } \\
\text { Behenic acid } \\
\text { Arachidic acid } \\
\text { Stearic acid }\end{array}$ & (65) \\
\hline
\end{tabular}




\begin{tabular}{|c|c|c|c|c|c|c|c|}
\hline Crop & QTL /Loci & MP & Cross(s) & Markers & Chromosome & $\begin{array}{l}\text { Nutritional parameters } \\
\text { in the grain }\end{array}$ & References \\
\hline & $\begin{array}{l}\text { S_mqPAb09_4, } \\
\text { S_mqAA_b098 } \\
\text { S_mqGA_b09_1 } \\
\text { S_mqSAa07_1 } \\
\text { S_mqBA_a09 } \\
\text { S_meLA_b08_2 }\end{array}$ & RILs & Sunoleic97R/NC94022 (Population S) & $\begin{array}{l}\text { FAD2B } \\
\text { RN34A10 } \\
\text { FAD2A } \\
\text { Seq2A06 }\end{array}$ & $\begin{array}{l}\text { B9 } \\
\text { A7 } \\
\text { A9 } \\
\text { B8 }\end{array}$ & $\begin{array}{l}\text { Palmatic acid, Arachidic } \\
\text { acid, Gadoleic acid } \\
\text { Stearic acid } \\
\text { Behenic acid } \\
\text { Lignoceric acid }\end{array}$ & (66) \\
\hline & $\begin{array}{l}\text { T_mqSA_b04_1, } \\
\text { T_mqAA)b04_1, } \\
\text { T_mqLA_b04_1 } \\
\text { T_mqGA_b04_1, T_mqBA_b04_4 } \\
\text { T_mqPA_a09_4 }\end{array}$ & RILs & Tifrunner/GTC20 (Population T) & $\begin{array}{l}\text { PM15 } \\
\text { TC4H07 } \\
\text { FAD2A }\end{array}$ & $\begin{array}{l}\text { B9 } \\
\text { B4 } \\
\text { A9 }\end{array}$ & $\begin{array}{l}\text { Stearic acid Arachidic } \\
\text { acid, } \\
\text { Lignoceric acid } \\
\text { Gadoleic acid, } \\
\text { Behenic acid } \\
\text { Palmatic acid }\end{array}$ & (66) \\
\hline & $\begin{array}{l}\text { mqQA181, } \\
\text { mqLA182, maOLR } \\
\text { maOC }\end{array}$ & RILs & Sunoleic97R/NC94022 (S population) & $\begin{array}{l}\text { ahFAD2B } \\
\text { GM1878 }\end{array}$ & $\begin{array}{l}\text { B9 } \\
\text { A5 }\end{array}$ & $\begin{array}{l}\text { Oleic acid, Linoleic acid, } \\
\text { Oleic/Linoleic acid } \\
\text { Oil content }\end{array}$ & (67) \\
\hline & $\begin{array}{l}\text { mqQA181, } \\
\text { mqLA182, mqOLR } \\
\text { mqOC }\end{array}$ & RILs & Tifrunner/GT-C2O (T population) & $\begin{array}{l}\text { ahFAD2A } \\
\text { GM2690-1 }\end{array}$ & $\begin{array}{l}\text { B9 } \\
\text { B8 }\end{array}$ & $\begin{array}{l}\text { Oleic acid, Linoleic acid, } \\
\text { Oleic/Linoleic acid } \\
\text { Oil content }\end{array}$ & (67) \\
\hline \multicolumn{2}{|c|}{ Mung beanqFe4.1, qZn6.4, } & RILs & ML776/Sattya & PVBR82 (SSR), CEDG248 (SSR) & 4,6 & $\mathrm{Fe}, \mathrm{Zn}$ & (68) \\
\hline & $\begin{array}{l}\text { SDPAP4.1 } \\
\text { SDIP10.1 } \\
\text { SDTP4.1 }\end{array}$ & $\mathrm{F}_{2}$ & V1725BG/Aus TRCF 321925 & $\begin{array}{l}\text { CEDG139-MB-SSR179 (SSR) } \\
\text { VR226-CEDG097 (SSR) } \\
\text { Bmd25-MB-SSR179 (SSR) }\end{array}$ & $\begin{array}{l}4 \\
10 \\
4\end{array}$ & $\begin{array}{l}\text { Low phytic acid } \\
\text { Inorganic phosphate } \\
\text { Total phosphorus }\end{array}$ & (69) \\
\hline \multirow[t]{4}{*}{ Soybean } & $\begin{array}{l}\text { qCys-7-2 } \\
\text { qMet-8-1 } \\
\text { qSAA-15-1 } \\
\text { qPC-8-1 }\end{array}$ & RILs & Kefeng no. 1 and Nannong 1138-2 & $\begin{array}{l}\text { Bin } 148(\mathrm{SNP}) \\
\text { Bin } 34(\mathrm{SNP}) \\
\text { Bin } 124(\mathrm{SNP}) \\
\text { Bin } 37(\mathrm{SNP})\end{array}$ & $\begin{array}{l}7 \\
8 \\
15 \\
8\end{array}$ & $\begin{array}{l}\text { Cysteine } \\
\text { Methionine } \\
\text { Sulfur containing amino } \\
\text { acids } \\
\text { Protein content }\end{array}$ & (70) \\
\hline & $\begin{array}{l}\text { qPRO001 } \\
\text { qOIL001 } \\
\text { qPAL002 } \\
\text { qOLE003 } \\
\text { qLIN001 } \\
\text { qGLU001 } \\
\text { qALA001 qCYS001 } \\
\text { qVAL001 } \\
\text { qHIS001 }\end{array}$ & RILs & Hamilton/Spencer & $\begin{array}{l}\text { ss249909538-ss249919445 } \\
\text { ss246100375-ss245879277 } \\
\text { ss245914593-ss245908292 } \\
\text { ss249909538-ss249506152 } \\
\text { ss245914593-ss245790648 } \\
\text { ss245914593-ss245908292 } \\
\text { ss246100375-ss245879277 } \\
\text { ss245898080-ss245908292 } \\
\text { ss249909538-ss249919445 } \\
\text { ss245898080-ss245908292 }\end{array}$ & $\begin{array}{l}18 / \mathrm{G} \\
6 / \mathrm{C} 2 \\
6 / \mathrm{C} 2 \\
18 / \mathrm{G} \\
6 / \mathrm{C} 2 \\
6 / \mathrm{C} 2 \\
6 / \mathrm{C} 2 \\
6 / \mathrm{C} 2 \\
18 / \mathrm{G} \\
6 / \mathrm{C} 2\end{array}$ & $\begin{array}{l}\text { Protein } \\
\text { Oil } \\
\text { Palmitic } \\
\text { Oleic } \\
\text { Linolenic } \\
\text { Glutamic } \\
\text { Alanine } \\
\text { Cysteine } \\
\text { Valine } \\
\text { Histidine }\end{array}$ & $(71)$ \\
\hline & qIF5-1 & RILS & Huachun 2/Wayro & Bin 799-800 (SNP) & 5 & Isoflavone content & (72) \\
\hline & $\begin{array}{l}\text { qProt_Gm20 and } \\
\text { qLsy_Gm20 } \\
\text { qThr_Gm20, qMet_Gm20 and } \\
\text { qMet+Cys_Gm20 } \\
\text { qCys_Gm10 }\end{array}$ & RILs & Benning/Danbaekkong & $\begin{array}{l}\text { GSM0012 (SNP)-satt354 (SSR) } \\
\text { GSM0012-BARC-020713 (SNP) } \\
\text { satt592 (SSR)-BARC043247 (SNP) }\end{array}$ & $\begin{array}{l}20 \\
20 \\
10\end{array}$ & $\begin{array}{l}\text { Protein content and } \\
\text { Lysine content } \\
\text { Threonine, Methionine and } \\
\text { Methionine + Cysteine } \\
\text { content } \\
\text { Cysteine content }\end{array}$ & d \\
\hline
\end{tabular}




\begin{tabular}{|c|c|c|c|c|c|c|c|}
\hline Crop & QTL /Loci & MP & Cross(s) & Markers & Chromosome & $\begin{array}{l}\text { Nutritional parameters } \\
\text { in the grain }\end{array}$ & References \\
\hline & suc1, suc3, suc2 & $\mathrm{F}_{3}$ & MFS-553/PI243545 & $\begin{array}{l}\text { ss245668753, ss249186914, } \\
\text { ss246796276 (SNP) }\end{array}$ & $5,9,16$ & Sucrose content & (74) \\
\hline & $q P C$ & RILS & 1.R05-1415/R05-638 2.V97-1346/R05-4256 & $\begin{array}{l}\text { satt451-satt614 (SSR) } \\
\text { ss250447161-ss250327854 (SNP) }\end{array}$ & $\begin{array}{l}20 \\
20\end{array}$ & Protein content & (75) \\
\hline & $q O C$ & RILs & R05-1415/R05-638 & satt451-satt614 (SSR) & 20 & Oil content & (76) \\
\hline & $\begin{array}{l}\text { qCa-8-1 } \\
\text { qMg-13 } \\
\text { qZn-11-1 } \\
\text { qFe-3 } \\
\text { qP-11 }\end{array}$ & RILs & Kefeng1/Nannong1138-2 & $\begin{array}{l}\text { sat_162-AW132402 } \\
\text { satt335-satt522 } \\
\text { satt251 } \\
\text { satt675-satt237 } \\
\text { satt197 }\end{array}$ & $\begin{array}{l}8 \\
13 \\
11 \\
3 \\
11\end{array}$ & $\begin{array}{l}\text { Calcium content } \\
\text { Magnesium } \\
\text { Zn } \\
\text { Fe } \\
\text { Phosphorous }\end{array}$ & (77) \\
\hline & $\begin{array}{l}\text { qPRO_B1 } \\
\text { qOIL_C1,J,O }\end{array}$ & RILs & $S_{02}-4-59 / A_{02}-381100$ & $\begin{array}{l}\text { BARCSoYSSR-17-0621 (SSR) } \\
\text { Sat_140, Sat_350, satt581 (SSR) }\end{array}$ & $\begin{array}{l}17 \\
4,16,10\end{array}$ & $\begin{array}{l}\text { Protein content } \\
\text { Oil content }\end{array}$ & (78) \\
\hline & $\begin{array}{l}\text { QPRO_13_1 } \\
\text { qPRO_13_1 }\end{array}$ & $\mathrm{F}_{2: 3}$ & $\begin{array}{l}\text { 1. Jidou 12/ZYD } 2738 \text { (G. soja) 2. Jidou 9/ZYD } \\
2738 \text { (G. soja) }\end{array}$ & $\begin{array}{l}\text { satt114 (SSR) } \\
\text { satt114 }\end{array}$ & 13 & Protein content & (79) \\
\hline & $\begin{array}{l}\text { qCys and qCys-Met } \\
\text { qMet }\end{array}$ & RILs & $\begin{array}{l}\text { 1.Williams } 82 \text { /DSR-173 } \\
\text { 2. Williams } 82 / N K S 19-90 \\
\text { 3. Williams } 82 \text { /Vinton } 81\end{array}$ & $\begin{array}{l}\text { BARC-038869-07364 - BARC-039753-07565 } \\
\text { (SNP) } \\
\text { BARC-018461-02916 - } \\
\text { BARC-066103-17539 (SNP) }\end{array}$ & & $\begin{array}{l}\text { Cysteine and Cystein }+ \\
\text { Methionine content } \\
\text { Methionine content }\end{array}$ & (80) \\
\hline & qIF20-2 & RILS & Luheidou2/Nanhuizao & M943408-M941848 (SLAF) & 20 & Isoflavone content & (81) \\
\hline & Ca5 & $\begin{array}{l}F_{2: 4} \\
F_{2: 5}\end{array}$ & $\begin{array}{l}\mathrm{PI} 408052 \mathrm{~B} / \mathrm{PI} 408052 \mathrm{C} \\
\text { PI408052B/KS 43035P }\end{array}$ & Sat_290- satt115 & 18 & Calcium content & (82) \\
\hline & $\begin{array}{l}\text { qPRO001 } \\
\text { qOILO08 } \\
\text { qPALO02 } \\
\text { qSTELO01 } \\
\text { qOLEO03 } \\
\text { qLINLOO3 } \\
\text { qLINNOO7 }\end{array}$ & RILs & MD96-5722/Spencer & $\begin{array}{l}\text { ss248293401-ss248275088 (SNP) } \\
\text { ss248308943-ss248309108 (SNP) } \\
\text { ss249629157-ss249621644 (SNP) } \\
\text { ss248977568-ss248979552 (SNP) } \\
\text { ss249037210-ss249039670 (SNP) } \\
\text { ss248981433-ss248993887 (SNP) } \\
\text { ss249010538-ss249039670 (SNP) }\end{array}$ & $\begin{array}{l}14 \\
14 \\
18 \\
16 \\
16 \\
16 \\
16\end{array}$ & $\begin{array}{l}\text { Protein content } \\
\text { Oil content } \\
\text { Palmatic acid Stearic acid } \\
\text { Oleic acid } \\
\text { Linoleic acid } \\
\text { Linolenic acid }\end{array}$ & (83) \\
\hline & $\begin{array}{l}\text { qPRO7-5 } \\
\text { QPRO20-1 }\end{array}$ & RILs & Charleston/Dongnong 594 & $\begin{array}{l}\text { satt358-Sat_001 (SSR) } \\
\text { satt331-satt173 (SSR) }\end{array}$ & $\begin{array}{l}7 \\
20\end{array}$ & Protein content & (84) \\
\hline \multirow[t]{3}{*}{$\begin{array}{l}\text { Brassica } \\
\text { napus }\end{array}$} & $\begin{array}{l}\text { qoil.1 } \\
\text { qpro-1 } \\
\text { qoil+pro-1 }\end{array}$ & $\mathrm{DH}$ & SGDH14/cv. Express & $\begin{array}{l}\text { pP12638473-p12699181 (SNP) } \\
\text { p18005556-p11609327 (SNP) } \\
\text { p12699181-scaff_17119_1_p115218 }\end{array}$ & $\begin{array}{l}\mathrm{A} 08 \\
\mathrm{~A} 07 \\
\mathrm{~A} 08\end{array}$ & $\begin{array}{l}\text { Oil content } \\
\text { Protein content } \\
\text { Oil and Protein content }\end{array}$ & (85) \\
\hline & $\begin{array}{l}\text { BnaA05g } 23520 \\
\text { BnaA05g } 23930\end{array}$ & $\mathrm{DHs}$ & RIL324/RIL622 & $\begin{array}{l}\text { UQSNP0001565 } \\
\text { UQSNP0001759 }\end{array}$ & & Oleic and Linoleic acid & (86) \\
\hline & $\begin{array}{l}\text { CqOC-A8-2 } \\
\text { uqFA-C3-3 } \\
\text { uqFA-A8-4 }\end{array}$ & $\mathrm{DH}$ & Tapidor × Ningyou 7 & & $\begin{array}{l}\text { A8 } \\
\text { C3 } \\
\text { A8 }\end{array}$ & $\begin{array}{l}\text { Oil content } \\
\text { C16:O/C18:0/C18:1/ } \\
\text { C18:2/C20:0/C20:1/ } \\
\text { C22:0/C22:1/C16:0/ } \\
\text { C18:0/C18:1/C18:2/ } \\
\text { C18:3/C20:0/C20:1/ } \\
\text { C22:0/C22:1/ }\end{array}$ & (87) \\
\hline
\end{tabular}


TABLE 1 | Continued

\begin{tabular}{|c|c|c|c|c|c|c|c|}
\hline Crop & QTL /Loci & MP & Cross(s) & Markers & Chromosome & $\begin{array}{l}\text { Nutritional parameters } \\
\text { in the grain }\end{array}$ & References \\
\hline & $\begin{array}{l}\text { Gene BrFAD5 } \\
\text { Gene BrFAD7 }\end{array}$ & $\mathrm{DHs}$ & YS 143 /Nai Bai Cai & $\begin{array}{l}\text { Bra027203 } \\
\text { Bra034863 }\end{array}$ & $\begin{array}{l}\text { A05 } \\
\text { A05 }\end{array}$ & $\begin{array}{l}\text { Erucic acid } \\
\text { Oleic and Linoleic acid }\end{array}$ & (88) \\
\hline & $\begin{array}{l}\text { qOIL-A10a } \\
\text { qC16:0-C8b } \\
\text { qC18:0-C3e, qC18:1-C3c, } \\
\text { qC18:2-C3c, } \\
\text { qC18:3-C3c, qC20:0-C3d }\end{array}$ & $\mathrm{DHs}$ & Polo 9/Topas & $\begin{array}{l}\text { BnGMS288-311/CB10536-163 } \\
\text { em1/bg9-434/bg23/pm59-285 } \\
\text { pm88/pm45-177/odd3/pm3-399 }\end{array}$ & $\begin{array}{l}\mathrm{A} 10 \\
\mathrm{C} 8 \\
\mathrm{C} 3\end{array}$ & $\begin{array}{l}\text { Oil content } \\
\text { Palmatic acid } \\
\text { Stearic, Oleic, Linoleic, } \\
\text { Linolenic, Arachidic acid }\end{array}$ & (89) \\
\hline & $\begin{array}{l}\text { Oil content } \\
\text { Protein content } \\
\text { Erucic acid and } \\
\text { Stearic acid } \\
\text { Linoleic acid } \\
\text { Glucosinolate }\end{array}$ & $\mathrm{DHs}$ & Tapidor/Ningyou7 & $\begin{array}{l}\text { Bn-scaff_23761_1-p249628 (SNP) } \\
\text { Bn-scaff_17119_1-p349622 (SNP) } \\
\text { Bn-scaff_15794_1-p347392 (SNP) } \\
\text { Bn-A02-p10850012 (SNP) } \\
\text { Bn-scaff_15794_1-p437864 (SNP) }\end{array}$ & $\begin{array}{l}\mathrm{C} 03 \\
\mathrm{CO} 3 \\
\mathrm{CO} 3 \\
\mathrm{~A} 02 \\
\mathrm{CO} 3\end{array}$ & $\begin{array}{l}\text { Oil content } \\
\text { Protein content } \\
\text { Erucic and } \\
\text { Stearic acid } \\
\text { Linoleic acid } \\
\text { Glucosinolate }\end{array}$ & (90) \\
\hline & OilC3-3 & DHs & Tapidor/Ningyou7 & Bn-scaff_23761_1-p249628 (SNP) & $\mathrm{CO3}$ & Oil content & (91) \\
\hline & $\begin{array}{l}\text { PRT.C6.w.1 } \\
\text { SUL.A2.w.1 } \\
\text { OLA.A9.w.1 } \\
\text { OIL.C3.w.1 } \\
\text { LIA.C3.w.1 } \\
\text { GSL.A2.w.1 } \\
\text { ERA.C3.w.1 }\end{array}$ & Inbreds & 405 Inbred lines & $\begin{array}{l}\text { Bn-ctg7180014756759-p1575 } \\
\text { Bn-ctg7180014748062-p8451 } \\
\text { Bn-Scaffold000110-p349432 } \\
\text { Bn-ctg7180014717095-p1564 } \\
\text { Bn-ctg7180014726380-p989 } \\
\text { Bn-ctg7180014748062-p8451 } \\
\text { Bn-ctg7180014717095-p1564 } \\
\text { (SNP markers) }\end{array}$ & $\begin{array}{l}\mathrm{C} 6 \\
\mathrm{~A} 2 \\
\mathrm{~A} 9 \\
\mathrm{C} 3 \\
\mathrm{C} 3 \\
\mathrm{~A} 2 \\
\mathrm{C} 3\end{array}$ & $\begin{array}{l}\text { Protein content } \\
\text { Sulphur content } \\
\text { Oleic acid } \\
\text { Oil content } \\
\text { Linolenic acid } \\
\text { Glucosinolate } \\
\text { Erucic acid }\end{array}$ & (92) \\
\hline & $\begin{array}{l}\text { qLysC-16-3 } \\
\text { qThrC-12-5 } \\
\text { qMetC-9-5 }\end{array}$ & $\mathrm{BC}$ & $\mathrm{BC}_{1} \mathrm{~F}_{1}$ (DHs/Tapidor) $\mathrm{BC}_{2} \mathrm{~F}_{1}$ (DHs/Ningyou 7$)$ & $\begin{array}{l}\text { HBR057/HBR047 } \\
\text { EM18ME6-220/NA12C03HBR096/IGF5385F }\end{array}$ & $\begin{array}{l}\text { C6 } \\
\text { C2 } \\
\text { A9 }\end{array}$ & $\begin{array}{l}\text { Lysine content } \\
\text { Threonine content } \\
\text { Methionine content }\end{array}$ & (93) \\
\hline & $\begin{array}{l}\text { qOC-2 } \\
q P C-1 \\
q O A C-2 \\
q G L C-1\end{array}$ & RILs & 827R/Darmor_Sin & $\begin{array}{l}\text { CB10369-220 - me5em16-170 } \\
\text { me1em1-400 - me4em7-400 } \\
\text { Olo9-A06-400 - me2em10-240 } \\
\text { me7em11-470 me8em20-230 } \\
\text { (SRAP markers) }\end{array}$ & $\begin{array}{l}11 \\
1 \\
12 \\
10\end{array}$ & $\begin{array}{l}\text { Oil content } \\
\text { Protein content } \\
\text { Oleic acid } \\
\text { Glucosinolate }\end{array}$ & (94) \\
\hline & $\begin{array}{l}q A 8-5 \\
q C 3-3\end{array}$ & $\mathrm{DHs}$ & Tapidor/Ningyou7 & $\begin{array}{l}\text { IGF1108c-sR7178 } \\
\text { IGF0235b-BRMS-093 }\end{array}$ & $\begin{array}{l}\text { A8 } \\
\text { C3 }\end{array}$ & $\begin{array}{l}\text { C16:0/C18:0/C18:1/ } \\
\text { C18:2/C18:3/C20:0/ } \\
\text { C20:1/C22:0/C22:1 }\end{array}$ & (95) \\
\hline & $\begin{array}{l}\text { qC16:0, qC18:1, qC18:2, } \\
\text { qC18:3, qC20:1 }\end{array}$ & $\mathrm{DHs}$ & Tapidor/Ningyou7 & HBr015 (A8) and JICB0633 (C3) & $\mathrm{A} 8$ and $\mathrm{C} 3$ & $\begin{array}{l}\text { C16:0, 18:1, C18:2, } \\
\text { C18:3, C20:1 }\end{array}$ & (96) \\
\hline & $F A D 3 A$ and $F A D 3 C$ genes & Inbreds & 21 Cultivars of winter and spring rape & \multicolumn{2}{|l|}{ LinAR-LinAF and LinCr-LinCF (dCAPS markers) } & Low Linolenic acid & (97) \\
\hline & BnFAD2-C5 & Inbred & Xiangyou & YG-C5-FAD2-F/YG-C5-FAD2-R & C5 & High oleic acid & (98) \\
\hline
\end{tabular}


TABLE 1 | Continued

\begin{tabular}{|c|c|c|c|c|c|c|c|}
\hline Crop & QTL /Loci & MP & Cross(s) & Markers & Chromosome & $\begin{array}{l}\text { Nutritional parameters } \\
\text { in the grain }\end{array}$ & References \\
\hline & $\begin{array}{l}\text { qOil_N19 } \\
\text { qC160180_N9 } \\
\text { qC181_N9 } \\
\text { qC182_N9 } \\
\text { qC183_N6 } \\
\text { qProtein_N16 }\end{array}$ & $\mathrm{DHs}$ & $\begin{array}{l}\text { DH12075 / } \\
\text { YN01-429 }\end{array}$ & $\begin{array}{l}- \\
- \\
- \\
- \\
-\end{array}$ & $\begin{array}{l}\text { N19 } \\
\text { N9 } \\
\text { N9 } \\
\text { N9 } \\
\text { N6 } \\
\text { N16 }\end{array}$ & $\begin{array}{l}\text { Oil content } \\
\text { C16:018:0 } \\
\text { C18:1 } \\
\text { C18:2 } \\
\text { C18:3 } \\
\text { Protein content }\end{array}$ & (99) \\
\hline & $\begin{array}{l}\text { qArgC-8-5 } \\
\text { qHisC-4-3 } \\
\text { qGluC-1-1 } \\
\text { qGlyC-4-1 } \\
\text { qProC-1-1 } \\
\text { qAlaC-7-3 } \\
\text { qAspC-9-4 }\end{array}$ & $\mathrm{BC}$ & $\begin{array}{l}\text { BC1F1 } 1 \text { (DHs/Tapidor) } \\
\text { BC1F1 } 2 \text { (DHs/'Ningyou7) }\end{array}$ & $\begin{array}{l}\text { HAU348/B034P14-1-1 } \\
\text { HS-k02-2/HBr094 } \\
\text { znS13M26-100/CB10081 } \\
\text { HBr094/CNU256 } \\
\text { znS13M26-100/CB10081 } \\
\text { znS13M26-340/JICB0571 } \\
\text { HBr075/JICB0516 }\end{array}$ & $\begin{array}{l}\text { A8 } \\
\text { A4 } \\
\text { A1 } \\
\text { A4 } \\
\text { A1 } \\
\text { A7 } \\
\text { A9 }\end{array}$ & $\begin{array}{l}\text { Arginine content } \\
\text { Histidine content } \\
\text { Glutamic acid } \\
\text { Glysine content } \\
\text { Protein content } \\
\text { Alanine content } \\
\text { Aspartic acid }\end{array}$ & $(100)$ \\
\hline & BnaFAD2 gene & Inbred & Tapidor & $\begin{array}{l}\text { BnaC.FAD2.a } \\
\text { BnaA.FAD2.b }\end{array}$ & $\begin{array}{l}\text { A5 } \\
\text { A1 }\end{array}$ & High Oleic and low PUFA & $(101)$ \\
\hline \multirow[t]{2}{*}{$\begin{array}{l}\text { Brassica } \\
\text { carinata }\end{array}$} & $\begin{array}{l}\text { qPRO10 } \\
\text { qEru.C4-1 } \\
\text { qLEN.C4-1 } \\
\text { qOLE.C4-1 } \\
\text { qLEI.B8-4 }\end{array}$ & $\mathrm{DHs}$ & Y-BcDH64/W-BcDH76 & $\begin{array}{l}100059607 \text { (DArTseq) } \\
100036778 \text { (DArTseq) } \\
100065508 \text { (DArTseq) } \\
100026342 \text { (DArTseq) } \\
100035893 \text { (DArTseq) }\end{array}$ & $\begin{array}{l}\text { B7 } \\
\text { C4 } \\
\text { C4 } \\
\text { C4 } \\
\text { B8 }\end{array}$ & $\begin{array}{l}\text { Protein content } \\
\text { Erucic acid } \\
\text { Linolenic acid } \\
\text { Oleic acid } \\
\text { Linoleic acid }\end{array}$ & (102) \\
\hline & $\begin{array}{l}\text { PRO-WH13 } \\
\text { OLE-WH14 } \\
\text { OC-WH13 } \\
\text { LEN-WH14 } \\
\text { ERU-WH13 }\end{array}$ & Inbreds & 81 diverse accessions & $\begin{array}{l}5121285 \text { (DArTseq) } \\
5859309 \text { (DArTseq) } \\
5121285 \text { (DArTseq) } \\
5863483-1 \text { (DArTseq) } \\
5121285 \text { (DArTseq) }\end{array}$ & $\begin{array}{l}\text { C8 } \\
\text { B2 } \\
\text { C8 } \\
\text { B3 } \\
\text { C8 }\end{array}$ & $\begin{array}{l}\text { Protein content } \\
\text { Oleic acid } \\
\text { Oil content } \\
\text { Linolenic acid } \\
\text { Erucic acid }\end{array}$ & $(102)$ \\
\hline \multirow[t]{2}{*}{$\begin{array}{l}\text { Brassica } \\
\text { juncea }\end{array}$} & $\begin{array}{l}\text { TGLC-S } \\
\text { GNA-S } \\
\text { SIN-S } \\
\text { IBE S } \\
\text { NEO-S } \\
\text { NAS-S } \\
\text { TOC } \\
\text { OIL }\end{array}$ & RILs & NUDH-YJ-04/RL-1359. & $\begin{array}{l}\text { SB3739a -SB3739b (SSR) } \\
\text { NA14B05- NIA138 (SSR) } \\
\text { CNU566- NA12D08 (SSR) } \\
\text { NIA010 -NIA046 (SSR) } \\
\text { NIA045 -NIA043 (SSR) } \\
\text { NA12H09- NA12G08 (SSR) } \\
\text { CNU111 -CNU483 (SSR) } \\
\text { NIA044- CNU402b (SSR) }\end{array}$ & $\begin{array}{l}\text { J18 } \\
\text { J1 } \\
\text { J4 } \\
\text { J9 } \\
\text { J7 } \\
\text { J4 } \\
\text { J6 } \\
\text { J9 }\end{array}$ & $\begin{array}{l}\text { Glucosinolate } \\
\text { Gluconapin } \\
\text { Sinigrin } \\
\text { Glucoiberin } \\
\text { Gluconeobrasscin } \\
\text { Gluconasturtin } \\
\text { Tocopherol } \\
\text { Oil content }\end{array}$ & (103) \\
\hline & $\begin{array}{l}\text { FAE1.1 } \\
\text { FAE1.2 }\end{array}$ & $\begin{array}{l}\text { Inbreds and } \\
\mathrm{BC}\end{array}$ & $\begin{array}{l}18 \text { Inbred lines PM24/Pusa Vijay PM30/Pusa } \\
\text { Bold }\end{array}$ & $\begin{array}{l}\text { CAPS591, CAPS1265 } \\
\text { CAPS237 }\end{array}$ & $\begin{array}{l}\text { A8 } \\
\text { B7 }\end{array}$ & Low erucic acid & (104) \\
\hline \multirow[t]{2}{*}{$\begin{array}{l}\text { Brassica } \\
\text { rapa }\end{array}$} & BnFAE1 gene & $\mathrm{BC}$ & Tori-7 / Kirariboshi & $\begin{array}{l}\text { BnFAE1.1-dcapsF } \\
\text { BnFAE1.1-3UTR-cR }\end{array}$ & $\begin{array}{l}\mathrm{A} \\
\mathrm{C}\end{array}$ & Low erucic acid & $(105)$ \\
\hline & $\begin{array}{l}\text { Gene BrFAD5 } \\
\text { Gene BrFAD7 }\end{array}$ & $\mathrm{DHs}$ & YS 143 /Nai Bai Cai & $\begin{array}{l}\text { Bra027203 } \\
\text { Bra034863 }\end{array}$ & $\begin{array}{l}\text { A05 } \\
\text { A05 }\end{array}$ & $\begin{array}{l}\text { Erucic acid } \\
\text { Oleic and linoleic acid }\end{array}$ & (88) \\
\hline $\begin{array}{l}\text { Brassica } \\
\text { oleracea }\end{array}$ & BoFAD3-2 gene & & alboglabra & $\begin{array}{l}\text { BoFAD3-2FY1 - BoFAD3-2FY2 (gene } \\
\text { specific markers) }\end{array}$ & & Low $\alpha$-linolenic acid & (106) \\
\hline
\end{tabular}




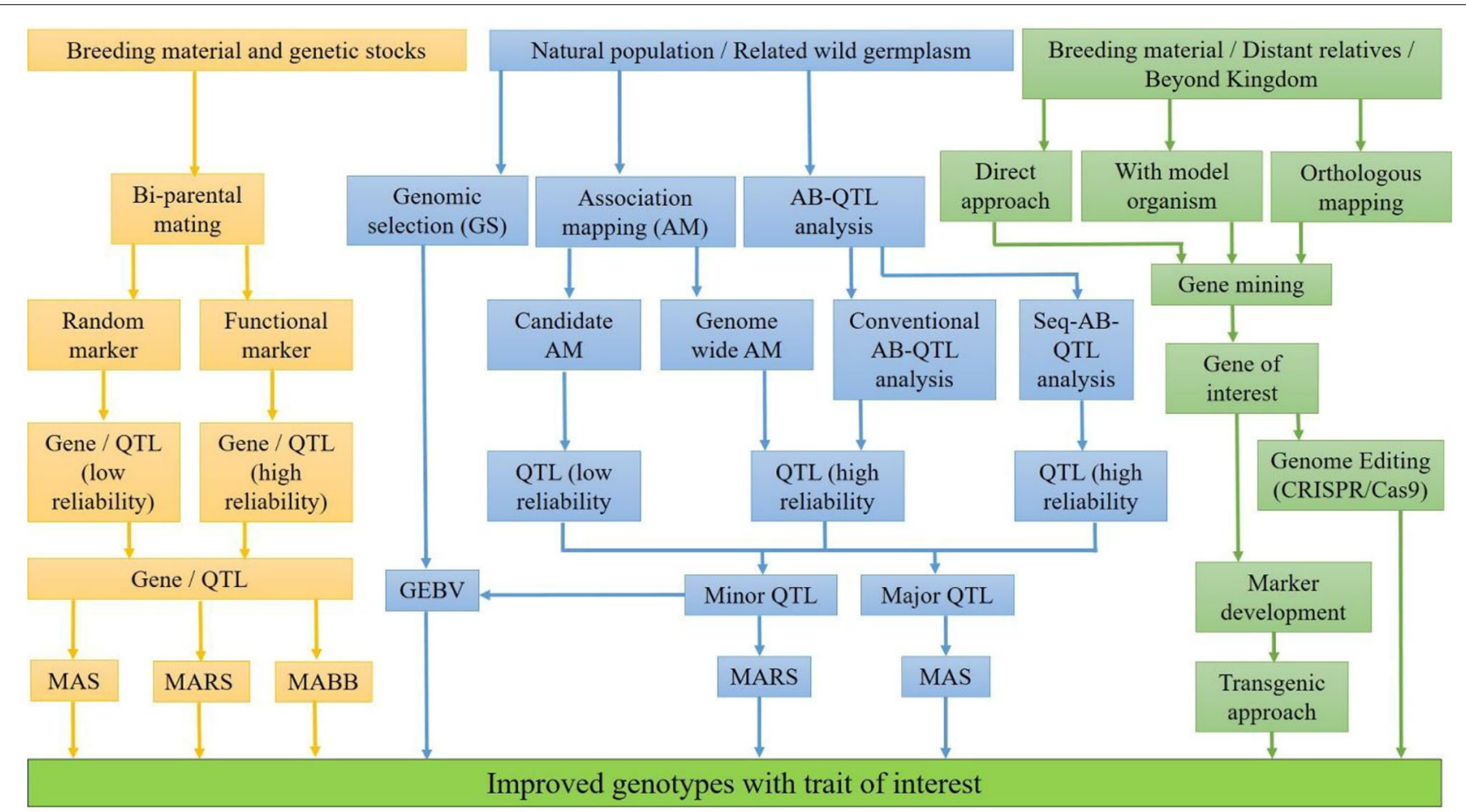

FIGURE 1 | Approaches for developing genotypes with enhanced nutritional traits.

A7, A8, and A9 (100). The details of the linked markers to these QTLs are given in Table 1.

\section{QTLs for Minor Elements}

Trace elements are required in a minimal amount, and these micronutrients are vital as they are often acting as catalysts in chemical reactions. In rice, QTLs, viz., qCo 1, qCu 2, qMn 7, and $q M o r$ in BILs for $\mathrm{Co}, \mathrm{Cu}, \mathrm{Mn}$, and $\mathrm{Mo}$ on chromosomes $1,2,5$, and 7, have been identified (41). Similarly, in maize, QTLs ( $q M n 1, q \mathrm{Co} 3, q \mathrm{Cu} 8, q \mathrm{~K} 4, q M o 1, q N a 5, q \mathrm{P} 4$, and $q S 1)$ for some trace elements $(\mathrm{Mn}, \mathrm{Co}, \mathrm{Cu}, \mathrm{K}, \mathrm{Mo}, \mathrm{Na}, \mathrm{P}$, and $\mathrm{S}$ ) have been identified on chromosomes 1, 3, 4, 5, and 8, respectively, by Baxter et al. (45). Furthermore, Ates et al. (56) identified four QTLs for selenium content using SNP markers in lentil (Table 1). QTLs [(K)—Ps5.1, (Mn)—Ps5.1, (Mo)-Ps5.1, and (P) - Ps3.1] for minor nutrients were identified through SSR markers in pea RILs (59). Sompong et al. (33) reported QTLs for low phytic acid (SDPAP4.1), inorganic phosphate (SDIP10.1), and total phosphorus (SDTP4.1) using SSR markers in mungbean. The details of the linked makers to these QTLs and QTLs linked to other minor elements are given in Table $\mathbf{1}$.

Improving the nutritional status of elite breeding lines requires functional information about the genetic network controlling important traits such as $\mathrm{Fe}, \mathrm{Zn}$, and protein content, oil content, pro-vitamin A, an array of amino acids, etc. Although impressive progress has been made in the identification of a large number of QTLs of nutritional traits in major food staples, an important challenge is their introgression into the agronomically superior background without compromising the yield and their popularization in malnourished areas.

\section{GENOMIC APPROACHES FOR ENHANCING NUTRITIONAL QUALITY IN MAJOR FOOD CROPS}

For the development of nutritionally rich cultivars, a suitable breeding strategy, available genetic diversity, and modern genomics information are needed. Sufficient genetic variability for nutritional quality traits has been explored in cultivars, direct progenitors, and wild relatives over the years. Moreover, a big treasurer of genetic diversity across the kingdoms can be explored and utilized through genetic engineering and genome editing. This diversity should be converted into nutritionally rich cultivars. Therefore, proper understanding of the genetic basis of nutritional quality traits and interaction with the environment is of utmost importance for an efficient breeding program. The integration of modern genomics, physiology coupled with precise phenotyping and advance breeding methodologies, has effectively revealed the genes and the metabolic pathways of quality traits. The identification and the incorporation of nutritional quality traits is being facilitated by several approaches (Figure 1), each having its own advantages and disadvantages.

The identification of loci for trait of interest can be facilitated with QTL mapping by the population developed from bi-parental mating or the natural population having large variability for the trait of interest. In bi-parental QTL mapping approach, two parents having a difference for the target trait are crossed 
to generate a mapping population of the mortal (F2, F3, etc.) or immortal (RILs, NILs, etc.) in nature. QTLs can also be identified with natural population with the help of association mapping (AM), viz., genome-wide AM, candidate-gene based $\mathrm{AM}$, and bulk segregant analysis (BSA). In genome-wide AM, polymorphism of the whole genome is considered at once for mapping of locus, while in candidate-gene based AM, the polymorphism of a particular genomic region for mapping of locus is considered. In BSA, target genomic regions linked with trait of interest are identified with simultaneous transfer from wild source into recipient during backcross program. The reliability of the identified QTLs depends on the closeness of the marker with the linked locus/QTLs. QTLs identified with functional markers/genic markers are more reliable because these markers are from the transcribed region and/or part of the identified locus. Therefore, SNP genotyping coupled with precise phenotyping, bioinformatics, and modern statistical algorithm specific to available data has enabled the identification of genomic regions controlling our traits of interest. Once genomic regions/QTLs associated with nutritional traits are identified, the next step is to utilize them for the development of nutritionally rich cultivars. The decision to transfer the target nutrional traits with molecular breeding depends on the relative eliteness and adaptation of the donor parent, the genetic complexity of the trait, the relative cost of phenotypic assays, the trait-linked marker assays, and genome profiling (119). If a nutritional trait is controlled by few loci, in elite genetic background, marker-assisted selection (MAS) is suggested. However, if the donor parents are landraces or distant relatives, marker-assisted backcross breeding (MABB) would be suitable for the transfer of trait. When the nutritional trait is controlled by a large number of genes with low genetic variance, genomic selection would be the choice to transfer the target trait (119). Genomic selection (GS) in crop breeding is gaining prominence due to the current availability of low-cost high-density genotyping with high-density DNA array chips and genotyping by sequencing (120, 121). In GS, all marker effects across the genome are estimated together by fitting the phenotypic and the marker data of the base training population with an appropriate statistical model (120). Then, genomic estimated breeding values (GEBVs) of any unknown genotypes having only marker information are generated with these marker effects. GEBVs predict the breeding values of unobserved genotypes for selection in the testing population $(122,123)$. With marker-assisted selection, nutritional traits like QPM and pro-vitamin A, controlled by major genes in maize, have been transferred in elite hybrids and released for commercial cultivation in India from ICARIARI, Delhi, India. These hybrids, namely, "Pusa Vivek QPM9 Improved" having high QPM and pro-vitamin A in 2017, "Pusa Vivek Hybrid-27 Improved" having high pro-vitamin A, and "Pusa HQPM5 Improved" and "Pusa HQPM7 Improved," both having high QPM and pro-vitamin A in 2019, have been released for commercial cultivation in India so far $(119,124)$. In bread wheat, some success stories, but not of commercial success, of the introgression of major gene Gpc-B1 (high protein content) with MAS have been reported by several researchers (125-129). In durum wheat, one successful example of a cultivar having high protein content using MAS, namely, "Desert KingHigh Protein," had been developed at the University of California wheat breeding program (130). However, little success has been achieved in the development of nutritionally rich commercial cultivars with MAS in other food crops. The simple reason is that most of these traits are controlled by a large number of genes having a little effect individually in the expression of these traits. In bread wheat, the major QTLs for Zn content, namely, QZn2A and QZn7B, were each having only $11.9 \%$ genetic variance (19); therefore, introgression of these QTLs with MAS into an elite background is very difficult. In addition, genotype by environment interaction increases the complexity many folds in the transfer of target traits. Therefore, for complex quantitative traits governed by many minor QTLs, genomic selection (GS) would be a better choice than MAS (131). As a matter of fact that the genes or major QTLs for nutritional qualities are known, there are large numbers of minor QTL effects on the expression of trait. To support this fact, the betaine aldehyde dehydrogenase $(\mathrm{BADH} 2)$ gene solely is not able to explain the variation of aroma in rice because background minor QTLs contribute to the overall fragrance in rice $(132,133)$. Therefore, integration of minor effect QTLs for nutritional traits can be effectively accomplished by employing the recurrent selection under breeding cycles under GS.

\section{Genome Editing Technologies for Enhancing Nutritional Quality in Major Food Crops}

Although conventional breeding is currently the most preferred and widely used approach for developing nutrient-enriched genotypes/varieties, it is labor-intensive and resource consuming, and it usually takes several years to develop a final product right from the screening of large germplasm lines, making crosses, and selection of desired recombinants that can finally turn into the commercial variety. Genetically modified (GM) crops that have beneficial traits are developed by the transfer of transgene(s) of known function into elite crop varieties. The transgenic approach can be an alternative for the development of biofortified crops when the genetic variation for a particular nutrient is limited or absent in the germplasm. Unlimited genetic variation across the boundary of species can be explored through this approach. However, their use is largely affected by unsubstantiated health and environmental safety concerns raised by non-governmental organizations and many developed countries. Government regulatory frameworks in many developed countries that aim to safeguard human and environmental biosafety have led to significant cost barriers to the rapid widespread adoption of new GM traits (134). As a result, the advantages of GM traits have been restricted to a small number of cultivated crops such as maize (high lysine), soybean (high oleic acid), potato (high amylopectin), cassava (high content of iron, $\beta$-carotene, and protein), and canola (phytate degradation) (3). Genome editing technologies facilitate efficient, precise, and targeted modifications at the genomic loci (135). The first-generation genome editing technologies 
that use ZFNs (136) and TALENs (137) have been around for two decades. They are labor-intensive, time-consuming, and involve a complex procedure to gain target specificity. However, second-generation genome editing techniques like CRISPR/Cas9 system (138) require less time and cost and provide simplicity and ease of targeted gene editing. All of these technologies use typical sequence-specific nucleases that can be induced to recognize specific DNA sequences and to generate double-stranded breaks (DSBs). The DSBs are repaired by plants' endogenous repair mechanisms, viz., nonhomologous end joining, which can lead to the insertion or deletion of nucleotides, thereby causing gene knockouts, or by homologous recombination, which can cause gene replacements and insertions (139). The risks involved in altering genomes through the use of genome editing technology are significantly lower than those associated with GM crops because most edits alter only a few nucleotides, producing changes that are not unlike those found throughout naturally occurring populations (140). Once the genomic editing agents have segregated out, there is no way to distinguish between a "naturally occurring" mutation and a gene edit. Thus, the introduction of genome editing into modern breeding programs should facilitate rapid and precise crop improvement. Genome editing techniques often produce gene knockout mutants, gene replacement, and insertion mutants, thus becoming a potent tool in the improvement of traits of nutritional quality in major food crops (Table 2).

The first-generation genome editing technique TALENs has been used for modifying and enhancing the nutritional profile of major food crops. Soybean oil contains high levels of polyunsaturated linoleic and linolenic acid, which contribute to oxidative instability. This problem is often addressed through partial hydrogenation. However, partial hydrogenation increases the levels of trans-fatty acids, which have been associated with cardiovascular disease. Altering the composition of soybean oil by increasing the level of oleic acid and decreasing the levels of linoleic and linolenic acids may help reduce the need for hydrogenation. Soybean lines with high oleic acid and low linoleic acid contents were generated by introducing mutations in the two fatty acid desaturase 2 genes (FAD2-1A and FAD2-1B) (149) and fatty acid desaturase $3 \mathrm{~A}(F A D 3 A)$ gene by directly delivering TALENs into fad21a fad2-1b soybean plants (150) and FAD2-2 gene using CRISPR/Cas9 (151), thus improving the shelf-life and heat stability of soybean oil. The same gene ahFAD2 was mutated for the isolation of high oleic acid lines in peanut using CRISPR/Cas9 (153).

Rice is a staple food crop feeding more than half of the world population. High amylose content and resistant starch improves human health and reduces the risk of serious diseases including hypertension, diabetes, and colon cancer (158). The CRISPR/Cas9 technology has been successfully used to create high-amylose rice by targeting two rice branching enzymes, $S B E I$ and SBEIIb (143). Rice bran oil (RBO) contains many valuable healthy constituents, including oleic acid. Fatty acid desaturase 2 (FAD2) catalyzes the conversion of oleic acid to linoleic acid in plants. To produce high oleic/low linoleic RBO, Abe et al.
(145) disrupted the OsFAD2-1 gene by CRISPR/Cas9-mediated targeted mutagenesis and developed rice lines with high oleic acid (twice that of the wild type) and low linoleic acid content. Enhancing the level of $\beta$-carotene is an important target of biofortification in major food crops because it is a precursor of pro-vitamin A. Endo et al. (146) were successful in accumulating $\beta$-carotene in rice callus (up to $2.86 \pm 1.41 \mu \mathrm{g} / \mathrm{g}$ fresh weight) by identifying the putative ortholog of the cauliflower or gene in rice, Osor, and modifying the Osor gene via genome editing using CRISPR/Cas9. The majority of the rice varieties grown over the world are having a white pericarp. However, rich diversity also exists for brown, red, or purple/black pericarp. The red pericarp contains high levels of proanthocyanidins and anthocyanins which have been recognized as health-promoting nutrients (159). The red coloration in the grains of wild rice is controlled by two complementary genes, $R c$ and $R d(160,161)$. Recently, Zhu et al. (147) successfully converted three elite white-pericarp rice varieties into red ones having high proanthocyanidin and anthocyanin content without compromising the yield potential through CRISPR/Cas9-mediated functional recovery of the recessive $\mathrm{rc}$ allele. Aromatic rice is popular worldwide for the characteristic fragrance of its grains. More than 100 volatile compounds were detected in the flavor of cooked fragrant rice. The presence of a defective badh2 allele encoding $\mathrm{BADH}$ 2 results in the synthesis of 2-acetyl-1-pyrroline, which is a major fragrance compound in aromatic rice. Shan et al. (144) reported the creation of fragrant rice from a non-fragrant variety via the targeted knockout of OsBADH2 using the TALEN method.

Using CRISPR/Cas9, DuPont Pioneer, in 2016, developed the first waxy corn hybrid by knocking out the maize waxy gene $W x 1$, making more than $97 \%$ amylopectin and essentially eliminating amylose from the kernel (148). The variety with high amylopectin starch content has higher digestibility and many industrial applications. The release of commercial hybrids with this trait is planned for 2020 (148). The researchers from Swedish Agricultural University developed high-amylopectin potatoes by knocking out the granule-bound starch synthase gene using CRISPR/Cas9 (154). The high-amylopectin potato starch has uses in both food and technical applications.

In potato tubers, the low temperature during cold storage stimulates the accumulation of reducing sugars that influence the quality of the product. Upon high-temperature processing, these reducing sugars react with free amino acids, resulting in brown, bitter-tasting products and elevated levels of acrylamide-a potential carcinogen. To minimize the accumulation of reducing sugars, vacuolar invertase (VInv) gene was knocked out by the TALENs technique (155).

The gluten protein of wheat which is responsible for the unique viscoelastic properties of wheat-derived foods also triggers gluten sensitivity in susceptible individuals commonly known as celiac disease. Because of the complexity of the Gli-2 locus and the high copy number of a-gliadin genes, conventional breeding and mutagenesis have failed to develop low-immunogenic wheat varieties for patients with celiac disease. Low-gluten, transgene-free wheat lines with much reduced immunoreactivity, using CRISPR/Cas9, have been developed 
TABLE 2 | Nutritional quality traits improved by genome editing technologies in major food crops.

\begin{tabular}{|c|c|c|c|c|}
\hline Crop species & Gene editing technology & Target gene & Trager trait & References \\
\hline Wheat & CRISPR/Cas9 & $\alpha$-gliadin gene family & Low gluten content & $(141)$ \\
\hline Wheat & CRISPR/Cas9 & $\alpha$-and $\gamma$-gliadin gene family & Low gluten content & $(142)$ \\
\hline Rice & CRISPR/Cas9 & SEBIlb & High amylose content & $(143)$ \\
\hline Rice & TALENs & OsBadh2 & Enhanced aroma & $(144)$ \\
\hline Rice & CRISPR/Cas9 & OsFAD2-1 & Oleic acid content & $(145)$ \\
\hline Rice & CRISPR/Cas9 & Osor & Enhanced $\beta$-carotene content & $(146)$ \\
\hline Rice & CRISPR/Cas9 & $R c$ & Proanthocyanidinis and anthocunins & $(147)$ \\
\hline Maize & CRISPR/Cas9 & $W \times 1$ & Waxy corn & $(148)$ \\
\hline Soybean & TALENS & FAD2-1A, FAD2-1B & High oleic acid content & $(149)$ \\
\hline Soybean & TALENs & FAD2-1A, FAD2-1B & High oleic, low linoleic acid content & $(150)$ \\
\hline Soybean & CRISPR/Cas9 & FAD2-2 & High oleic, low linoleic acid content & $(151)$ \\
\hline Soybean & TALENs & GmPDS11 and GmPDS18 & Carotenoid biosynthesis & $(152)$ \\
\hline Peanut & CRISPR/Cas9 & ahFAD2 & High oleic acid content & $(153)$ \\
\hline Potato & CRISPR/Cas9 & $W \times 1$ & High amylopectin & $(154)$ \\
\hline Potato & TALENs & VInv & Low reducing sugars & $(155)$ \\
\hline Rapeseed & CRISPR/Cas9 & FAD2 & High oleic acid content & $(156)$ \\
\hline Rapeseed & CRISPR/Cas9 & BnaFAD2 & High oleic acid content & $(157)$ \\
\hline
\end{tabular}

$(141,142)$ and will serve as donors for introgressing low gluten trait into elite wheat varieties.

Modification of fatty acid composition is one of the primary objectives in the improvement of oilseed brassicas. The fatty acid desaturase 2 gene, FAD2, is a key gene that affects oleic, linoleic, and linolenic acids. The CRISPR/Cas9-mediated genome editing system has been applied for developing lines with high oleic acid (more than $80 \%$ ) of rapeseed (Brassica napus) $(156,157)$ and in the emerging oilseed plant Camelina sativa $(162,163)$ by modifying the FAD2 gene. In yet another study in Camelina, the content of very long fatty acids was reduced by knocking out the FAE1 gene (164).

Genome editing technologies, because of their efficiency, high specificity, and amenability to multiplexing, have increasingly become popular genomic tools for enhancing the nutritional value of our major food crops. The mutant line generated through CRISPR/Cas9 will provide new genetic diversity for the traits of agronomic and nutritional importance for breeding in an unprecedented way.

\section{Developing Nutrient-Enriched Varieties Through Conventional and Genomics-Assisted Breeding}

Developing nutrient-enriched varieties of food crops through conventional plant breeding is the most preferred approach. The improvement of a particular trait requires the availability of sufficient and useful genetic diversity. This genetic diversity may be present in cultivated genotypes or can be introgressed from wild/related/progenitor species or can be created through directed mutagenesis. Work on the development of nutrientrich varieties of major food crops is ongoing in various public and private sector organizations with the support of important programs like HarvestPlus, Global Alliance for Improved Nutrition (GAIN), Project ENABLE (Expanding
Nutrition Access by Building capacity, Linking Initiative and Enhancing policy), HEALTHGRAIN Forum, etc. The purpose of all these programs is to improve the nutritional status of the food crops and make them available for all the people, especially the most vulnerable ones. Conventional breeding supported by the above-mentioned programs has yielded several nutrientenriched varieties in important food crops (Table 3).

\section{Wheat}

Wheat is a staple cereal crop worldwide and one of the most preferred food crops for biofortification. The major target in wheat biofortification is the improvement of iron $(\mathrm{Fe})$ and zinc $(\mathrm{Zn})$ content in the grain. Wheat is blessed with a wider genetic variation for these two traits contributed by wild/progenitor species. This rich genetic diversity introgressed from Triticum durum-, Triticum spelta-, and Triticum dicoccum-based synthetic hexaploid wheat (SHW) into cultivated bread wheat led to the development of four high-Zn varieties in India and Pakistan. In India, the variety "Zinc Shakti" (Chitra), with $14 \mathrm{ppm}$ $\mathrm{Zn}$, was developed through participatory variety selection and further registered by private seed companies and growers. This variety has profitable yield potential and matures nearly 2 weeks earlier than common wheat. Two more varieties, viz., "WB 02" and "HPBW 01," were developed in 2017 by ICAR-India Institute of Wheat and Barley Research (ICAR-IIWBR) and Punjab Agricultural University (PAU), Ludhiana, respectively, for cultivation in the North-Western Plain Zone (NWPZ) of India (166) (Table 3). Wheat variety HD 3171, developed by ICARIndian Agricultural Research Institute (ICAR-IARI), New Delhi, is shown to have high iron content (47.2 ppm) (167). In Pakistan, the variety Zncol 2016 with +9 ppm $\mathrm{Zn}$ has been released for commercial cultivation (166) (Table 3). Apart from SHW, variation for high zinc content is present in wheat germplasm and varieties. In 2018, Bangladesh has witnessed the release of the first biofortified blast-resistant variety "Bari Gom 33" with +7 
TABLE 3 | Nutrient-enriched important biofortified crops developed through conventional and genomics-assisted breeding.

\begin{tabular}{|c|c|c|c|c|c|}
\hline Crop & $\begin{array}{l}\text { Biofortified } \\
\text { nutrient }\end{array}$ & Name of variety & Country & $\begin{array}{l}\text { Developing } \\
\text { institute }\end{array}$ & References/source \\
\hline \multirow[t]{12}{*}{ Wheat } & Zinc and iron & WB 02 & India & $\begin{array}{l}\text { ICAR-IIWBR, India, } \\
\text { and CIMMYT }\end{array}$ & (165) \\
\hline & & HPBW 01 & India & $\begin{array}{l}\text { PAU, India, and } \\
\text { CIMMYT }\end{array}$ & (166) \\
\hline & Iron & HD 3171 & India & ICAR-IARI, India & (167) \\
\hline & Zinc & Zncol 2016 & Pakistan & CIMMYT & (166) \\
\hline & Zinc & BARI Gom 33 & Bangladesh & $\begin{array}{l}\text { Bangladesh Wheat } \\
\text { and Maize Research } \\
\text { Institute, and } \\
\text { CIMMYT }\end{array}$ & (166) \\
\hline & Zinc & $\begin{array}{l}\text { BHU } 1 \\
\text { BHU } 3 \text { (Akshay) } \\
\text { BHU } 5 \\
\text { BHU } 6\end{array}$ & India & $\begin{array}{l}\text { Banaras Hindu } \\
\text { University, CIMMYT, } \\
\text { and HarvestPlus }\end{array}$ & (168) \\
\hline & & PBW 757 & India & PAU, India & (170) \\
\hline & $\begin{array}{l}\text { Yellow pigment } \\
\text { (carotene) }\end{array}$ & $\begin{array}{l}\text { HI } 8627 \\
\text { HI } 8759 \\
\text { HI } 8777\end{array}$ & India & ICAR-IARI, India & (170) \\
\hline & $\begin{array}{l}\text { Anthocyanin } \\
\text { (colored wheat) }\end{array}$ & Scorpion & Austria & $\begin{array}{l}\text { Crop Research } \\
\text { Institute, Prague }\end{array}$ & (171) \\
\hline & & PS Karkulka & Slovak Republic & $\begin{array}{l}\text { National Agricultural } \\
\text { and Food Center }\end{array}$ & (172) \\
\hline & & $\begin{array}{l}\text { NABIMG } 9 \text { NABIMG } \\
10 \text { NABIMG } 11\end{array}$ & India & $\begin{array}{l}\text { National Agri-Food } \\
\text { Biotechnology } \\
\text { Institute, New Delhi }\end{array}$ & (173) \\
\hline & & Indigo & Austria & $\begin{array}{l}\text { BOKU-University of } \\
\text { Natural Resources } \\
\text { and Life Sciences, } \\
\text { Austria }\end{array}$ & (174) \\
\hline \multirow{3}{*}{ Rice } & Zinc & $\begin{array}{l}\text { BRRldhan } 62 \\
\text { BRRldhan } 64 \\
\text { BRRldhan } 72\end{array}$ & Bangladesh & $\begin{array}{l}\text { Bangladesh Rice } \\
\text { Research Institute } \\
\text { (BARI) and } \\
\text { HarvestPlus }\end{array}$ & $(177)$ \\
\hline & Zinc & CR Dhan 45 & India & $\begin{array}{l}\text { National Rice } \\
\text { Research Institute, } \\
\text { India }\end{array}$ & (178) \\
\hline & Protein & CR Dhan 310 & India & $\begin{array}{l}\text { National Rice } \\
\text { Research Institute, } \\
\text { India }\end{array}$ & (178) \\
\hline \multirow{2}{*}{$\begin{array}{l}\text { Maize: Quality } \\
\text { Protein Maize } \\
\text { (QPM) }\end{array}$} & $\begin{array}{l}\text { Lysine and } \\
\text { tryptophan }\end{array}$ & $\begin{array}{l}\text { Protina (composite) } \\
\text { Ratan (composite) }\end{array}$ & India & GBPUAT, Pantnagar & (179) \\
\hline & & $\begin{array}{l}\text { Shakti (composite) } \\
\text { Shakti1 } \\
\text { Shaktiman } 1 \\
\text { Shaktiman } 2 \\
\text { Shaktiman } 3 \\
\text { Shaktiman } 4\end{array}$ & India & $\begin{array}{l}\text { Indian Institute of } \\
\text { Maize Research } \\
\text { (IIMR), India }\end{array}$ & (179) \\
\hline
\end{tabular}


TABLE 3 | Continued

\begin{tabular}{|c|c|c|c|c|c|}
\hline Crop & $\begin{array}{l}\text { Biofortified } \\
\text { nutrient }\end{array}$ & Name of variety & Country & $\begin{array}{l}\text { Developing } \\
\text { institute }\end{array}$ & References/source \\
\hline & & $\begin{array}{l}\text { HQPM } 1 \\
\text { HQPM } 5 \\
\text { HQPM } 7\end{array}$ & India & $\begin{array}{l}\text { CCS Haryana } \\
\text { Agricultural University } \\
\text { (CCSHAU), Hisar, } \\
\text { India }\end{array}$ & (179) \\
\hline & & Vivek QPM 9 & India & $\begin{array}{l}\text { ICA-VPKAS Almora, } \\
\text { India }\end{array}$ & (179) \\
\hline & & $\begin{array}{l}\text { QPHM } 200 \text { and } \\
\text { QPHM } 300\end{array}$ & Pakistan & $\begin{array}{l}\text { National Agricultural } \\
\text { Research System } \\
\text { and CIMMYT }\end{array}$ & (180) \\
\hline & & $\mathrm{GH}-132-2$ & Ghana & $\begin{array}{l}\text { Agricultural Research } \\
\text { Centers Ethiopia and } \\
\text { CIMMYT }\end{array}$ & \\
\hline & & $\begin{array}{l}\text { BR-451 (OPV) } \\
\text { BR-473 (OPV) Assum } \\
\text { Preto (OPV) }\end{array}$ & Brazil & $\begin{array}{l}\text { University of Ghana } \\
\text { and CIMMYT }\end{array}$ & \\
\hline & & HB-PROTICTA & Guatemala & $\begin{array}{l}\text { Guatemala's Institute } \\
\text { for Agricultural } \\
\text { Science and } \\
\text { Technology and } \\
\text { CIMMYT }\end{array}$ & \\
\hline & & $\begin{array}{l}\text { Obatampa } \\
\text { Espoir }\end{array}$ & Burkina Faso & CIMMYT, Mexico & \\
\hline & & Obatampa & Cameroon & CIMMYT, Mexico & \\
\hline & & Obangaina (OPV) & Uganda & CIMMYT, Mexico & \\
\hline & & $\begin{array}{l}\text { HQ INTA-993 } \\
\text { NB-Nutrinta (OPV) }\end{array}$ & Nicaragua & CIMMYT, Mexico & \\
\hline & & EV 99 QPM & Nigeria & CIMMYT, Mexico & \\
\hline & & Ev 99 QPM & Togo & CIMMYT, Mexico & \\
\hline & & Lishe-K1 & Tanzania & CIMMYT, Mexico & \\
\hline & & $\begin{array}{l}\text { EV } 99 \text { QPM } \\
\text { DMRESR WQPM } \\
\text { Susuma }\end{array}$ & Senegal & CIMMYT, Mexico & \\
\hline & & $\mathrm{HQ}-31$ & Honduras & CIMMYT, Mexico & \\
\hline & & HQ-61 & El Salvador & CIMMYT, Mexico & \\
\hline & & ICA & Colombia & CIMMYT, Mexico & \\
\hline
\end{tabular}


TABLE 3 | Continued

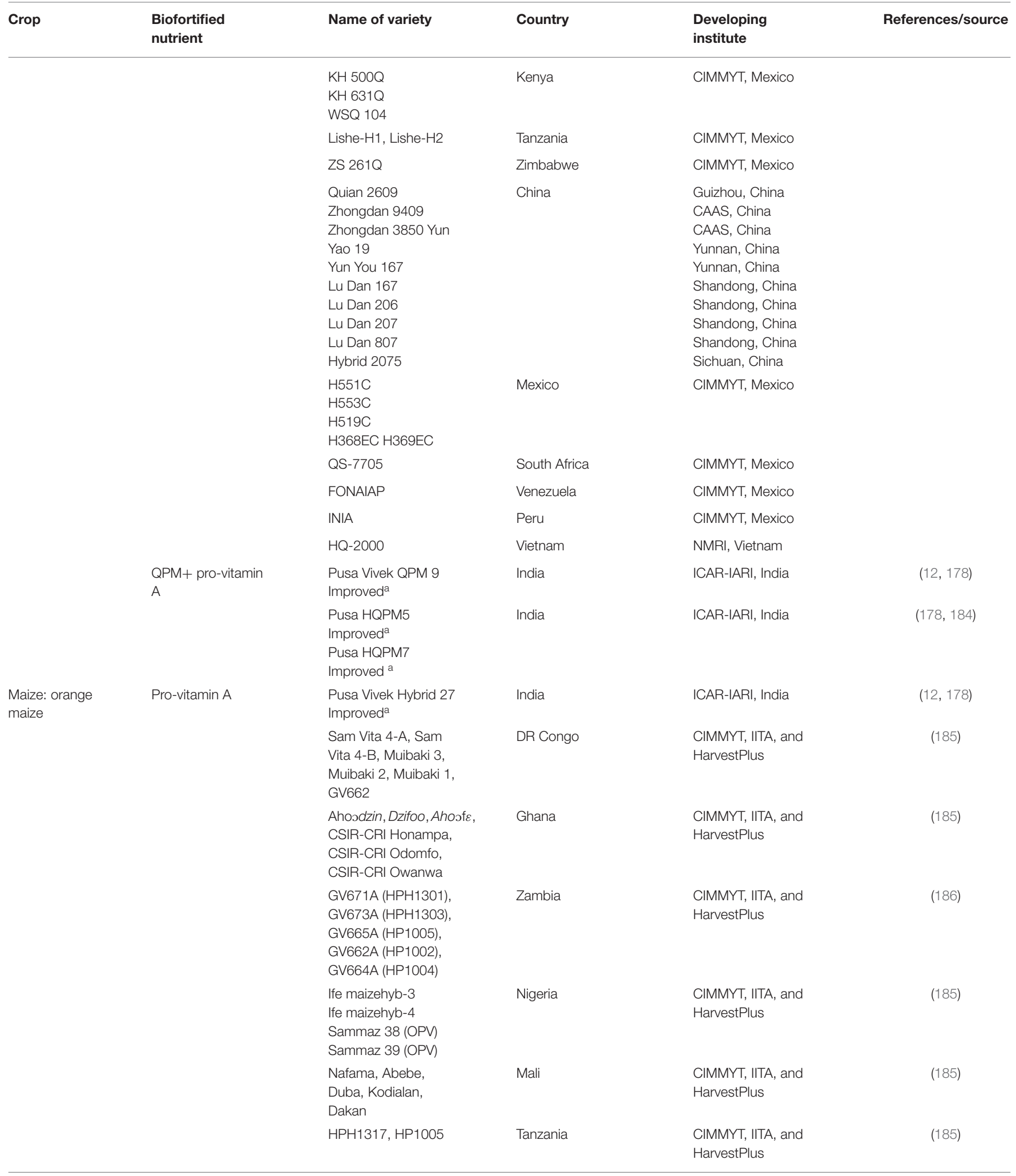


TABLE 3 | Continued

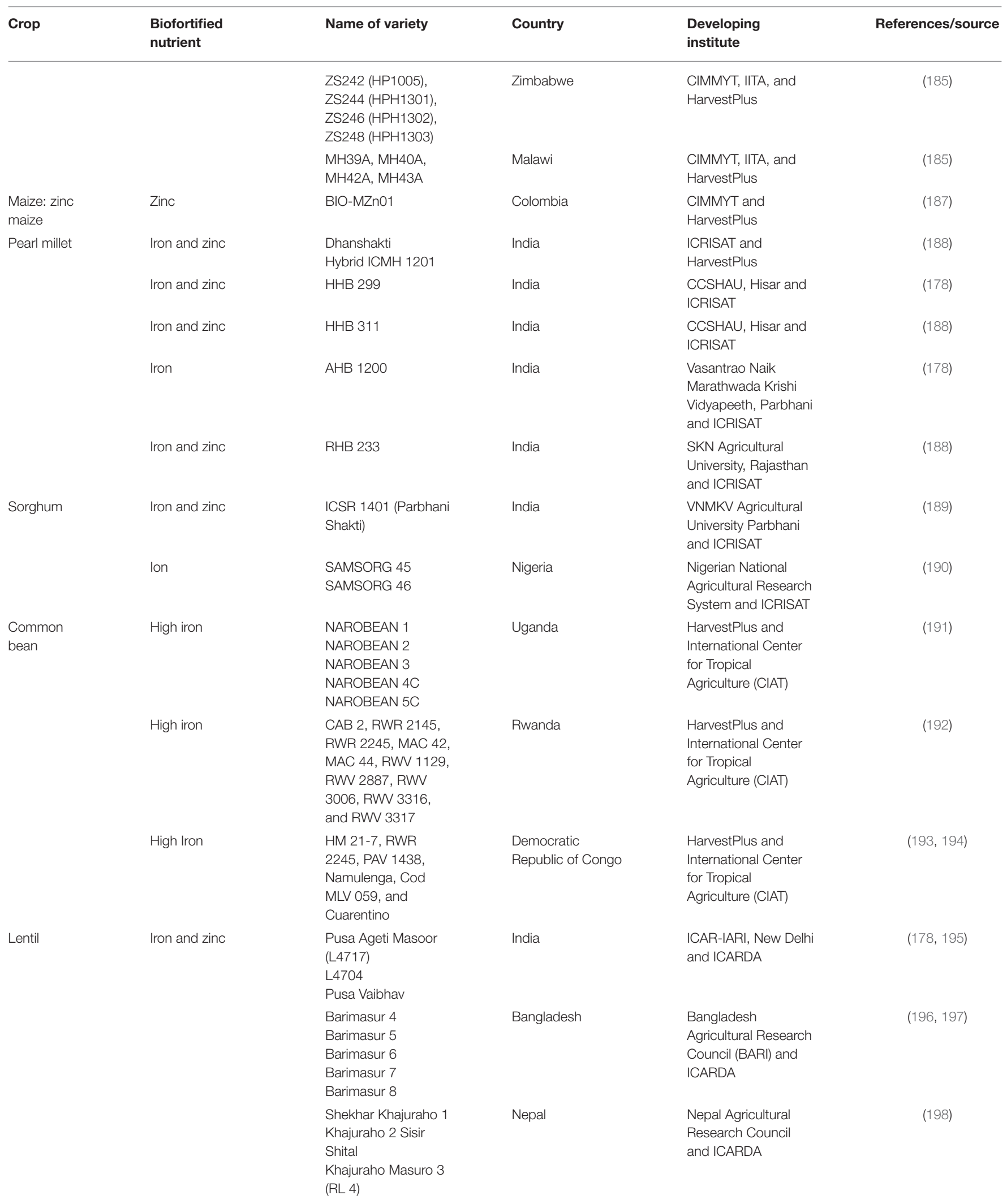


TABLE 3 | Continued

\begin{tabular}{|c|c|c|c|c|c|}
\hline Crop & $\begin{array}{l}\text { Biofortified } \\
\text { nutrient }\end{array}$ & Name of variety & Country & $\begin{array}{l}\text { Developing } \\
\text { institute }\end{array}$ & References/source \\
\hline & & Alemaya & Ethiopia & ICARDA & (199) \\
\hline & & Myveci-2001 & Turkey & ICARDA & \\
\hline & & Beleza & Portugal & ICARDA & \\
\hline Soybean & $\begin{array}{l}\text { Kuntiz trypsin } \\
\text { inhibitor (KTI)-free }\end{array}$ & $\mathrm{NRC} 127^{\mathrm{a}}$ & India & $\begin{array}{l}\text { ICAR-Indian institute } \\
\text { of Soybean } \\
\text { Research, India }\end{array}$ & (200) \\
\hline & & UEL $175^{a}$ & Brazil & $\begin{array}{l}\text { Sate University of } \\
\text { Londrina, Parana, } \\
\text { Brazil }\end{array}$ & (201) \\
\hline & $\begin{array}{l}\mathrm{KTI} \text { and } \\
\text { lipoxygenase } 2 \text { free }\end{array}$ & NRC $142^{a}$ & India & $\begin{array}{l}\text { ICAR-Indian institute } \\
\text { of Soybean } \\
\text { Research, India }\end{array}$ & (202) \\
\hline
\end{tabular}

a Varieties/hybrids developed through genomics-assisted breeding.

ppm $\mathrm{Zn}$ advantage, developed with the support of International Maize and Wheat Improvement Center (CIMMYT). In 2012, the HarvestPlus program of CGIAR, in alliance with Banaras Hindu University, India, and CIMMYT, developed high-Zn genotypes designated as BHU 1, BHU 3 (Akshay), BHU 5, and BHU 6. Through public-private partnership, HarvestPlus has reached more than 50,000 wheat farmers in the Eastern Gangetic Plain of India (168) (Table 3). Improvement in protein content is also an important target in wheat breeding. In general, protein content is believed to be negatively correlated with grain yield. However, in 2018, ICAR-Indian Agricultural Research Institute (IARI) New Delhi and PAU Ludhiana become successful in developing high-protein $(\sim 13 \%)$ and high-yielding bread varieties HD 3226 (169) and PBW 757, respectively, for NWPZ of India (170). Improvement of $\beta$-carotene content is a major target in durum wheat breeding program. The presence of yellow pigment in the grain generally measured as the Yellow Pigment Index (YPI), which is also a measure of $\beta$-carotene content, is an important quality trait and indicates an antioxidant in durum wheat. The bright yellow color of pasta due to the presence of yellow pigment in the flour is an indicator of good pasta quality. In India, durum wheat varieties such as WH 896, PDW 233, HI 8759, and HI 8777 with YPI $>19$ are developed and commercialized (170). In a wheat breeding program, one of the significant aims is to develop non-traditional genotypes having an added value which would impart a relevant health benefit. This biofortified colored wheat with high anthocyanin content is an extensive area of research. Anthocyanin can act as an antioxidant that removes harmful free radicals from the body and helps in the prevention of heart diseases, diabetes, obesity, and cancer. A winter wheat variety "Scorpion," with blue grain color, was registered in 2011 in Austria and in 2012 in Europe (171). Another winter wheat variety with purple grain "PS Karkulka" was developed by the National Agricultural and Food Center, Slovak Republic, in 2014 (172). In India, anthocyanin-rich wheat genotypes, viz., NABIMG 9, NABIMG 10, and NABIMG 11, with enhanced Zn content have been developed and registered (173) (Table 3).

\section{Rice}

The majority of the Asian population prefers rice as their staple food. This milled and polished rice is a poor source of some minerals and proteins; therefore, it becomes a preferred crop for biofortification. Gregorio et al. (176) screened improved cultivars, new plant type lines, local landraces, and lines from wild/related species for $\mathrm{Fe}$ and $\mathrm{Zn}$ content. It was reported that Fe $(15.6 \mathrm{mg} / \mathrm{kg})$ and $\mathrm{Zn}(378 \mathrm{mg} / \mathrm{kg})$ content was higher in wild/related species. They also reported that an Indian floating rice local cultivar "Jalmagna" had $40 \%$ more Zn concentration than the mega variety IR 64. An improved line (IR 68144-3132-2-3) with high grain Fe concentration (21 ppm in brown rice) was developed by the International Rice Research Institute. This line was derived from a cross of IR 72 and "Zawa Bonday," a traditional variety from India (176). In 2015, Bangladesh Rice Research Institute (BARI) has released their third high-Zn rice variety "BRRI dhan 72" with the support of HarvestPlus (177) (Table 3). Two earlier varieties, viz., BRRI dhan 62 (2013) and BRRI dhan 64 (2014), were also enriched with $\mathrm{Zn}$. The Indian Council of Agricultural Research (ICAR) has one of the mandates to improve the nutritional quality in high-yielding varieties of cereals, pulses, oilseeds, vegetables, and fruit crops. In rice, India has released two biofortified varieties of rice, viz., "CR Dhan 310 ” having high protein content (10.3\% in polished rice), which was developed by the National Rice Research Institute, Cuttack, and "DRR Dhan 45," having high Zn content (22.6 ppm) in polished grain, developed by the Indian Institute of Rice Research Hyderabad (178) (Table 3).

\section{Maize}

Maize is the third most important food grain, following wheat and rice. In recent years, the pro-vitamin A-enriched maize hybrids/varieties developed across the globe tell a success story of the biofortification program. In India, maize hybrid Pusa Vivek Hybrid Improved, enriched in pro-vitamin A, has been released for commercial cultivation (12). In Zimbabwe, orange maize rich in pro-vitamin A is bred especially for human consumption. The 
CIMMYT with HarvestPlus has also released biofortified orange maize variety "ZS 422" for commercial cultivation in Zimbabwe. Similarly, in many African countries where malnutrition is a serious concern, biofortified orange maize varieties have been released $(185,186)$ (Table 3$)$. Maize has a significant limitation; it lacks two essential amino acids, lysine and tryptophan, which are not synthesized in the human body. Maize breeders E. Villegas and SK Vasal at CIMMYT had developed quality protein maize (QPM) genotypes with high lysine and tryptophan by incorporating "opaque 2" gene along with genetic modifiers. In recent years, India has released a good number of QPM hybrids for commercial cultivation which includes Ratan, Protina, Shakti, Shakti 1, Shaktiman 1, Shaktiman 2, Shaktiman 3, Shaktiman 4, Shaktiman 5, HQPM 1, HQPM 5, HQPM 7, Vivel QPM 9, Vivek QPM 21, and Pratap QPM hybrid 1 with enhanced endospermic content of lysine and tryptophan (179) (Table 3). In addition to this, new QPM hybrids, viz., Pusa HM 4 Improved, Pusa HM 8 improved, and Pusa HM 9 Improved, with 0.68$1.06 \%$ lysine and $2.97-4.18 \%$ tryptophan were released by IARI, New Delhi, in 2017 (124) (Table 3). India released its first provitamin A-enriched QPM hybrid "Pusa Vivek QPM 9 Improved” with high pro-vitamin A (8.5 ppm), lysine (2.76 ppm), and tryptophan $(0.74 \%)$ (178). In the year 2020, IARI, New Delhi, again came up with three more hybrids, viz., Pusa HQPM 5 Improved and Pusa HQPM 7 Improved, having high pro-vitamin A in the QPM hybrid background, and one hybrid Pusa Vivek Hybrid 27 Improved, having high pro-vitamin A (200) (Table 3). The Global Maize Program of CIMMYT is the frontrunner in developing and releasing QPM hybrids for commercial cultivation in developing countries. The CIMMYT has also released QPM hybrids in Pakistan (QPHM 200 and QPHM 300), Ethiopia (BHQPY 545 and BHQP 542), Ghana (GH132-28), Guatemala (HB-PROTICTA), Nicaragua (HQ INTA993), Honduras (HQ-31), El Salvador (HQ-61), Colombia (ICA), Kenya (KH 500Q, KH 631Q), Tanzania (Lishe-H1, Lishe-H2), Ethiopia (Melkassa 1Q, Melkassa 6Q, MHQ 138), Zimbabwe (ZS 261Q), and China (Quian 2609, Zhong Dan 9409) (Table 3). Thus, QPM can be a better alternative to eliminate malnutrition in developing countries such as African nations where maize is a staple diet. In 2018, CIMMYT has released a new zincenriched maize variety, BIO-MZn01, in Colombia to help combat malnutrition in South America. This variety contains 36\% more zinc on average than other maize varieties, with grain yield of 6-8 t/ha (187). Furthermore, QPM combined with high Fe and $\mathrm{Zn}$, if developed, will be a favorable choice to tackle the problem of malnutrition.

\section{Pearl Millet}

Anemia is a significant health concern in millions of women and children in developing countries, especially in African and Asian countries. The reason for this is their staple diet which is deficient in iron (Fe). The pearl millet variety "Shakti" officially released in African country Niger and developed by International Crop Research Institute for Semi-Arid and Tropics (ICRISAT) has higher $\mathrm{Fe}(65 \mathrm{mg} / \mathrm{kg})$ and $\mathrm{Zn}$ (icrisat.org). In India, high$\mathrm{Fe}(75 \mathrm{mg} / \mathrm{kg})$ and high-Zn $(40 \mathrm{mg} / \mathrm{kg})$ variety "Dhanashakti" and hybrid "ICMH 1201" have been released by ICRISAT during
2012-2014 (Table 3). Recently, ICRISAT, in collaboration with state agricultural universities in India, has developed and released four biofortified hybrids, viz., AHB 1200 (iron 73.0 ppm), HHB 299 (iron 73.0 ppm; zinc 41.0 ppm), and HHB 311 and RHB 233 with high Fe and $\mathrm{Zn}$ content (178) (Table 3). These biofortified varieties and hybrids have 7.5 to $8.0 \mathrm{mg}$ of iron and 3.5 to $4.5 \mathrm{mg}$ zinc per 100 gm of pearl millet. It is estimated that consumption of $200 \mathrm{~g} /$ day biofortified pearl millet-based food will provide $70 \%$ of dietary Fe and $\mathrm{Zn}$ requirement in men and women, and $130 \mathrm{~g}$ /day will give $100 \%$ requirement for children (188). With these improvements, pearl millet is becoming 'Smart Food Crop' on account of its high $\mathrm{Fe}$ and $\mathrm{Zn}$ content, improved levels of drought, heat, and salinity tolerance, high protein with balanced amino acids, high dietary fibers, and gluten-free protein.

\section{Sorghum}

Sorghum is the grain of the twenty first century for Africa. It is the only viable food grain for millions of food-insecure people. In Nigeria, the Nigerian National Agricultural Research System and ICRISAT have released two sorghum varieties, "SAMSORG 45" and "SAMSORG 46," with 129 ppm Fe content. These biofortified varieties are higher yielding and resistant to Striga. In India, during 2018, ICRISAT has developed sorghum variety ICSR 14001 (later named as Parbhani Shakti), with 45 ppm $\mathrm{Fe}$ and $32 \mathrm{ppm} \mathrm{Zn}$. Besides these, it has low phytate content ( $4.14 \mathrm{mg} / 100 \mathrm{~g})$, which will increase the bioavailability of nutrients (Table 3 ).

\section{Common Bean}

Common bean is important for nutrient and poverty alleviation in the developing countries of Central America, Andean regions of South America, and Eastern and Southern Africa (203). Common bean has wide variation for Fe content, i.e., up to $110 \mathrm{ppm}$, which is much higher than the target of $40 \mathrm{ppm}$ set by HarvestPlus. Therefore, developing Fe-rich varieties through breeding is quite easy. The HarvestPlus and International Center for Tropical Agriculture (CIAT) have released five varieties (NAROBEAN 1, NAROBEAN 2, NAROBEAN 3, NAROBEAN 4C, and NAROBEAN 5C) in Uganda, 10 varieties (CAB 2, RWR 2145, RWR 2245, MAC 42, MAC 44, RWV 1129, RWV 2887, RWV 3006, RWV 3316, and RWV 3317) in Rwanda, and six varieties (HM 21-7, RWR 2245, PAV 1438, Namulenga, Cod MLV 059, and Cuarentino) in the Democratic Republic of Congo (191-194) (Table 3).

\section{Lentil}

Lentil is a highly nutritious pulse crop, well-adapted to dry land, in poor-soil-fertility areas of Africa, Middle-East, Indian-SubContinent, Southern Europe, America, Australia, and West Asia (204). Lentil has been a choice in priority for biofortification of mainly Fe, Zn, and Se. The International Center for Agricultural Research in Dry Areas (ICARDA) is working with national programs in India, Bangladesh, and Nepal to breed Fe- and $\mathrm{Zn}$-enriched lentil varieties. In recent years, ICARDA's research partnership with Bangladesh Agricultural Research Council (BARI) led to the release of five Fe- and Zn-enriched lentil varieties, namely, Barimasur 4, Barimasur 5, Barimasur 6, 
Barimasur 7, and Barimasur 8 (196, 197) (Table 3). These biofortified lentil varieties have reached 8,20,000 farmers in Bangladesh. In Nepal, five varieties (Shekhar, Khajuraho 1, Khajuraho 2, Sisir, and Shital) with 81-98 ppm Fe and $>54$ ppm $\mathrm{Zn}$ are rapidly gaining acceptance and popularity among 4,00,000 farmers (198). In India, three biofortified Fe-rich lentil varieties "Pusa Vaibhav" (102 ppm), IPL 220 (73-114 ppm), and L 4704 are becoming popular among the farmers of North Eastern India $(178,195)$ (Table 3).

\section{Soybean}

Elimination of Kunitz trypsin inhibitor (KTI) from soybean seeds is one of the important breeding objectives. For its inactivation, preheating of soy flour is required before using it in food and feed products. The heat treatment not only increases the processing but also affects the seed protein quality and solubility (205). For the development of KTI-free soybean lines, MABB approach was adopted to introgress the kti allele (source PI542044) in the genetic background of popular varieties in India: DS9712, DS9814(204), and JS97-52 (206). At ICAR-Indian Institute of Soybean Research, India, a soybean variety NRC 142, free from KTI and lipoxygenase 2, has been developed through marker-assisted breeding (https://iisrindore.icar.gov.in/pdfdoc/ NRC142News.pdf). In Brazil, a KTI-free variety UEL 175 has also been developed through marker-assisted breeding (201).

\section{CONVENTIONAL AND GENOMIC-ASSISTED BREEDING: ADVANTAGES AND LIMITATIONS}

Biofortification is targeted mainly to the rural poor in developing countries who are mainly dependent on staple foods for meeting their energy and nutrient demand and have very remote access to nutrient-rich fortified foods. The design of conventional plant breeding programs capitalizes on the available variability in the germplasm to breed nutrient-rich crops. The breeding for biofortification has been successful as several varieties have been released in different crops and are in cultivation in India, Pakistan, Zambia, etc. Both conventional and genomics-assisted breeding programs capitalize on the existing variability in the germplasm to breed nutrient-rich crops. The main advantage of both breeding strategies is that the varieties developed through these approaches have no yield penalty from an economic point of view and are also equally competitive to the earlier varieties in cultivation. The acceptability of varieties developed does not have an issue involved as in the case of genetically modified crops. Catering the nutrients through biofortification will uplift the livelihood as well as the nutritional status of the target population as this system is cost-effective and highly sustainable. Most of the quality traits are quantitative in nature and are highly influenced by environmental factors, because of which the progress of breeding varieties will be slow through conventional plant breeding. In many cases, the primary gene pool has limited variability; then, transferring the target trait from wild relatives poses serious constraints with respect to time duration and linked unfavorable genes (linkage drag). A large number of QTLs linked to key nutrient traits have been identified in different crops, which are amenable for marker-assisted selection, but in crops having a large genome size, like wheat, finding closely linked genes to the target traits was challenging, but with the advent of novel genomic tools, like genome-wide association mapping, genomic selection and also whole genome sequence data finding markers tightly linked to the trait of interest are possible. Markerassisted selection can be applied to transfer the genes/QTLs once closely linked markers are available. Numerous studies have been reported in literature regarding mapping of QTLs linked to different biofortification traits. By using genomicsassisted breeding, the time duration for the development of biofortified varieties can be reduced to 5 to 6 years as compared to conventional breeding, and the problem of linkage drag is also addressed in genomics-assisted breeding while transferring the target trait from wild relatives.

However, both of these approaches have some limitations; for example, if the target micronutrient does not have variability in the crop germplasm, then genetic improvement is impossible. In some cases, it would be impossible to breed for a specific trait using conventional means, and the timescale and the effort involved may be quite unrealistic, e.g., improving Se concentration in wheat grains (207). Rice plants possess the whole pathway to synthesize $\beta$-carotene; this pathway is fully active in leaves but turned off in the grain. By adding only two genes, a plant phytoene synthase (psy) and a bacterial phytoene desaturase (crt I), the pathway is turned back on and $\beta$-carotene consequently accumulates in the grain. On the other hand, it is not possible either through conventional or genomics-assisted breeding to switch on the pathway. In marker-assisted breeding, the linkage between the trait and the marker determines the success of a biofortification program. In some cases, the genetic background can alter the expression of the transferred traits while transferring QTLs. In case of cross-pollinated crops, the biofortified hybrids may lose the trait in one or few generations of inbreeding, so the farmers have to buy a fresh hybrid seed to maintain the required trait. In most of the developing countries, there is no demarcation or segregated procurement with respect to normal or biofortified wheat crop after harvest. So, all wheats get equal treatment with respect to the procurement price. If there is a scope for the segregated procurement of biofortified crops to be linked with some monetary benefit, such will encourage the adoptability of biofortified crops. Given the widespread prevalence of micronutrient deficiencies, conventional breeding coupled with the integration of genomic tools will be effective in providing access to micronutrientrich food to the target deficient population in developing countries as they capitalize on staple food crops for the delivery of micronutrients.

\section{PERSPECTIVES OF ENHANCING NUTRIENTS WITH CONVENTIONAL AND GENOMICS-ASSISTED BREEDING}

Increasing the content of nutritional elements/minerals through genetic biofortification has been demonstrated in major food 
crops, and several varieties have been released (Table 3). The traits governing nutritional contents are mostly polygenic and involved GX E interaction; hence, improvement through conventional plant breeding is possible, provided that the time required for the release of the variety is generally long. This is a sustainable and cost-effective solution for the elimination of malnutrition. Advancements in molecular genetics have aided in precise mapping and dissecting the molecular basis of biofortification. Several major-effect and stable QTLs for biofortification traits, such as GPC, minerals, and pigments, have been identified in different crops (Table 1). These QTLs can be transferred into elite lines using markerassisted selection, provided that the markers are tightly linked to the trait of interest, which is also sometimes challenging in crops having a large genome size. The advent of genomic selection has led to drastically reduced cost and increased throughput of genotyping assays, in combination with advances in high-throughput phenotyping. Through genomic selection, several QTLs pertaining to nutritional traits have been mapped and combined into elite lines in wheat (20). Increasing the micronutrients through genetic and genomic approaches is well-demonstrated where genetic variation exists in the germplasm. However, if genetic variation is not available in the germplasm, then novel techniques of genome editing (Table 2) and genetic engineering need to be employed, as demonstrated by establishing the pro-vitamin A pathway in golden rice (208). Golden rice has proved to elevate the pro-vitamin A level in rice, but due to strict GMO regulations and unexpected health risks, transgenic crops still have not found acceptance. Otherwise, golden rice has ample to offer to the world's malnutritionprone population.

The other less explored nutritional elements, like low sugar and omega 3- fatty acids, are gaining importance with the upsurge of chronic illnesses like diabetes and cardiovascular diseases. The challenge is to alter the starch structure of rice to lower its digestibility so that it provides a slow and steady supply of energy but without sacrificing its cooking and eating quality. A low-glycemic-index (GI) rice variety with hard texture is not preferred in the Southeast Asian market, where soft and sticky types are the benchmarks of rice quality. Variations in rice germplasm for low GI along with hard and cohesive texture have been assessed using genome-wide association mapping and are currently being used as pre-breeding materials to develop low-GI lines targeted toward certain market segments, particularly in Asia (209). Omega-3 fatty acids have proven to be very essential for human health due to their multiple health benefits like skin and hair growth as well as for proper visual, neural, and reproductive functions of the body, but the concern is that they are not produced by the human body. The requirement of these fatty acids is generally met by deep sea fishes as variation in plants is not that much. The entire DHA biosynthetic pathway was reconstituted in oilseed crop Brassica juncea by stepwise metabolic engineering. Transgenic plants produced up to $25 \%$ arachidonic acid and $15 \%$ eicosapentaenoic acid (EPA), as well as up to $1.5 \%$ docosahexaenoic acid (DHA) in seeds (210). However, the accumulation of EPA and DHA was low in transgenic lines, and therefore it was difficult to commercialize. An emerging oilseed crop, Camelina sativa seed, contains $>30 \%$ alpha-linolenic acid, which is the starting substrate required for the synthesis of EPA and DHA, making it a good platform for assembling the $\omega$-3-LC-PUFA synthesis pathway. The co-expression of five genes (OtD6 desaturase from the eukaryotic microalgae Ostreococcus tauri, TcD5 desaturase from marine fungus Thraustochytrium sp, Piw-3 desaturase from Phytophthora infestans, PsD12 desaturase from Phytophthora sojae, and D6 fatty acid carbon chain elongation enzyme PSE1 from Physcomitrella patens) has demonstrated EPA accumulation of up to $31 \%$ in camelina oil (211). The contents of EPA and DHA in this transgenic camelina were comparable to the levels found in fish oil, representing the successful assembly of biosynthetic pathways in commercial oil crop seeds, but there are regulatory issues in the case of transgenics. Genome-wide analysis tools have revealed several genomic regions associated with fatty acid composition in seed oil that had never before been implicated in lipid metabolism (212). By mining sequence data, the candidate enzymes for target fatty acids were identified and subsequently used to manipulate $C$. sativa oil composition toward a superior biofuel and bio-based lubricant oil $(213,214)$.

In view of this, conventional and genomics-assisted breeding both provide a way of improving the micronutrient status of crops as they have no acceptance problem like the transgenics. An additional advantage that these approaches offer is that farmers can use and retain the seed for food as well as for the next crop plantation. Staple crop biofortification will offer the advantage of delivering the much-needed micronutrients through seeds directly to the malnuorished population in the developing world. Additionally, further studies are needed to improve the bioavailability of these elevated protein, mineral, and pigment concentrations, and the target micronutrients for each crop need to be set by a nutrition specialist in consultation with breeders. The most important issue is making the farmers and populations aware about the nutritional benefits of consuming biofortified foods for their acceptability among the masses. With the immense potential and scope of biofortification, it can be envisaged that this technology can breed nutrientdense crops that will be crucial in addressing the problem of malnutrition.

\section{CONCLUSION}

Making our staple food crops nutrient-rich either by enriching our soils with nutrients or making nutrient-rich crops by modern breeding and biotechnological tools such as discovery and mapping of QTLs and their use in marker-assisted breeding will certainly help in eliminating malnutrition to a larger extent. The advanced QTL mapping tools like GWAS and the annotated whole-genome sequence of wheat and other cereals offer new opportunities to study the exact nature of allelic variation and explore the underlying genetic basis and putative gene(s) associated with quality traits. In conclusion, the compiled information on the QTLs on different nutrients flanked by linked marker systems, viz., SSR, SNP, DArT, and CAPS, would help in their precise introgression into elite breeding lines/cultivated 
varieties through MAS in biofortification breeding programs. Impressive work has been done in the identification of QTLs pertaining to nutritional quality and the release of nutritionally rich varieties through conventional breeding in major food crops. However, new avenues in genome editing research are making impressive findings in enriching our food crops to make them nutritionally rich. Genome editing technologies that can rapidly modify genomes in a precise way and will directly enrich the nutritional status of elite varieties could hold a bright future to address the challenge of malnutrition.

\section{AUTHOR CONTRIBUTIONS}

KG prepared the outline of the contents and coordinated the drafting of the manuscript. KG, SR, MK, VG, PB, and RY wrote the manuscript. NB assisted in final editing and addressing the comments. All authors contributed to the edits and the review.

\section{CONSENT FOR PUBLICATION}

The manuscript has been approved by all authors.

\section{REFERENCES}

1. Food and Agriculture Organization of the United Nations. The State of Food Insecurity in the World 2014: Strengthening the Enabling Environment for Food. Security and Nutrition. Food \& Agriculture Org. (2015).

2. Field CB, Barros V, Stocker TF, Dahe Q. Managing the Risks of Extreme Events and Disasters to Advance Climate Change Adaptation: Special Report of the Intergovernmental Panel on Climate Change. Cambridge University Press (2012).

3. Garg M, Sharma N, Sharma S, Kapoor P, Kumar A, Chunduri V, et al. Biofortified crops generated by breeding, agronomy, and transgenic approaches are improving lives of millions of people around the world. Front Nutr. (2018) 5:12. doi: 10.3389/fnut.2018.00012

4. White PJ, Broadley MR. Biofortifying crops with essential mineral elements. Trends Plant Sci. (2005) 10:586-93. doi: 10.1016/j.tplants.2005.10.001

5. Myers SS, Zanobetti A, Kloog I, Huybers $\mathrm{P}$, Leakey AD, Bloom AJ, et al. Increasing CO 2 threatens human nutrition. Nature. (2014) 510:13942. doi: $10.1038 /$ nature 13179

6. Loladze I. Hidden shift of the ionome of plants exposed to elevated CO2 depletes minerals at the base of human nutrition. elife. (2014) 3:e02245. doi: 10.7554/eLife.02245

7. Myers SS, Wessells KR, Kloog I, Zanobetti A, Schwartz J. Effect of increased concentrations of atmospheric carbon dioxide on the global threat of zinc deficiency: a modelling study. Lancet Glob Health. (2015) 3:e63945. doi: 10.1016/S2214-109X(15)00093-5

8. Palmer AC, Healy K, Barffour MA, Siamusantu W, Chileshe J, Schulze KJ, et al. Provitamin A carotenoid-biofortified maize consumption increases pupillary responsiveness among Zambian children in a randomized controlled trial. J Nutr. (2016) 146:2551-8. doi: 10.3945/jn.116.239202

9. Talsma EF, Brouwer ID, Verhoef H, Mbera GN, Mwangi AM, Demir AY, et al. Biofortified yellow cassava and vitamin A status of Kenyan children: a randomized controlled trial. Am J Clin Nutr. (2016) 103:25867. doi: 10.3945/ajen.114.100164

10. Babu R, Rojas NP, Gao S, Yan J, Pixley K. Validation of the effects of molecular marker polymorphisms in LcyE and CrtRB1 on provitamin A concentrations for 26 tropical maize populations. Theor Appl Genet. (2013) 126:389-99. doi: 10.1007/s00122-012-1987-3

11. Azmach G, Gedil M, Menkir A, Spillane C. Marker-trait association analysis of functional gene markers for provitamin A levels across diverse tropical yellow maize inbred lines. BMC Plant Biol. (2013) 13:227. doi: $10.1186 / 1471-2229-13-227$

12. Muthusamy V, Hossain F, Thirunavukkarasu N, Choudhary M, Saha S, Bhat JS, et al. Development of $\beta$-carotene rich maize hybrids through markerassisted introgression of $\beta$-carotene hydroxylase allele. PLoS ONE. (2014) 9:e113583. doi: 10.1371/journal.pone.0113583

13. Liu L, Jeffers D, Zhang Y, Ding M, Chen W, Kang MS, et al. Introgression of the crtRB1 gene into quality protein maize inbred lines using molecular markers. Mol Breed. (2015) 35:154. doi: 10.1007/s11032-015-0349-7

14. Yan J, Kandianis CB, Harjes CE, Bai L, Kim E-H, Yang X, et al. Rare genetic variation at Zea mays crtRB1 increases $\beta$-carotene in maize grain. Nat Genet. (2010) 42:322-7. doi: 10.1038/ng.551

15. Guo Y, Zhang G, Guo B, Qu C, Zhang M, Kong F, et al. QTL mapping for quality traits using a high-density genetic map of wheat. PLoS ONE. (2020) 15:e0230601. doi: 10.1371/journal.pone.0230601

16. Liu J, Wu B, Singh RP, Velu G. QTL mapping for micronutrients concentration and yield component traits in a hexaploid wheat mapping population. J Cereal Sci. (2019) 88:57-64. doi: 10.1016/j.jcs.2019.05.008

17. Fatiukha A, Filler N, Lupo I, Lidzbarsky G, Klymiuk V, Korol AB, et al. Grain protein content and thousand kernel weight QTLs identified in a durum $\times$ wild emmer wheat mapping population tested in five environments. Theor Appl Genet. (2020) 133:119-31. doi: 10.1007/s00122-019-03444-8

18. Gorafi YS, Ishii T, Kim J-S, Elbashir AAE, Tsujimoto H. Genetic variation and association mapping of grain iron and zinc contents in synthetic hexaploid wheat germplasm. Plant Genet Resour. (2018) 16:917. doi: $10.1017 / \mathrm{S} 1479262116000265$

19. Velu G, Singh RP, Crespo-Herrera L, Juliana P, Dreisigacker S, Valluru R, et al. Genetic dissection of grain zinc concentration in spring wheat for mainstreaming biofortification in CIMMYT wheat breeding. Sci Rep. (2018) 8:13526. doi: 10.1038/s41598-018-31951-Z

20. Velu G, Tutus Y, Gomez-Becerra HF, Hao Y, Demir L, Kara R, et al. QTL mapping for grain zinc and iron concentrations and zinc efficiency in a tetraploid and hexaploid wheat mapping populations. Plant Soil. (2017) 411:81-99. doi: 10.1007/s11104-016-3025-8

21. Crespo-Herrera L, Velu G, Singh R. Quantitative trait loci mapping reveals pleiotropic effect for grain iron and zinc concentrations in wheat. Ann Appl Biol. (2016) 169:27-35. doi: 10.1111/aab.12276

22. Tiwari C, Wallwork H, Arun B, Mishra VK, Velu G, Stangoulis J, et al. Molecular mapping of quantitative trait loci for zinc, iron and protein content in the grains of hexaploid wheat. Euphytica. (2016) 207:56370. doi: 10.1007/s10681-015-1544-7

23. Srinivasa J, Arun B, Mishra VK, Singh GP, Velu G, Babu R, et al. Zinc and iron concentration QTL mapped in a Triticum spelta $\times$ T. aestivum cross. Theor Appl Genet. (2014) 127:1643-51. doi: 10.1007/s00122-0142327-6

24. Hao Y, Velu G, Peña RJ, Singh S, Singh RP. Genetic loci associated with high grain zinc concentration and pleiotropic effect on kernel weight in wheat (Triticumaestivum L.). Mol Breed. (2014) 34:1893902. doi: 10.1007/s11032-014-0147-7

25. Sadeghzadeh B, Rengel Z, Li C. Quantitative trait loci (QTL) of seed Zn accumulation in barley population clipper X Sahara. J Plant Nutr. (2015) 38:1672-84. doi: 10.1080/01904167.2014.991036

26. Islam M, Arifuzzaman M, Banik S, Hossain M, Ferdous J, Khalequzzaman $M$, et al. Mapping QTLs underpin nutrition components in aromatic rice germplasm. PLoS ONE. (2020) 15:e0234395. doi: 10.1371/journal.pone.0234395

27. Pradhan S, Pandit E, Pawar S, Naveenkumar R, Barik S, Mohanty S, et al. Linkage disequilibrium mapping for grain $\mathrm{Fe}$ and $\mathrm{Zn}$ enhancing QTLs useful for nutrient dense rice breeding. BMC Plant Biol. (2020) 20:57. doi: 10.1186/s12870-020-2262-4

28. Jang S, Han J-H, Lee YK, Shin N-H, Kang YJ, Kim C-K, et al. Mapping and validation of QTLs for the amino acid and total protein content in brown rice. Front Genet. (2020) 11:240. doi: 10.3389/fgene.2020.00240 
29. Dixit S, Singh UM, Abbai R, Ram T, Singh VK, Paul A, et al. Identification of genomic region (s) responsible for high iron and zinc content in rice. Sci Rep. (2019) 9:8136. doi: 10.1038/s41598-019-43888-y

30. Zhong M, Wang LQ, Yuan DJ, Luo LJ, Xu CG, He YQ. Identification of QTL affecting protein and amino acid contents in rice. Rice Sci. (2011) 18:187-95. doi: 10.1016/S1672-6308(11)60026-7

31. Kinoshita N, Kato M, Koyasaki K, Kawashima T, Nishimura T, Hirayama Y, et al. Identification of quantitative trait loci for rice grain quality and yieldrelated traits in two closely related Oryza sativa L. subsp. japonica cultivars grown near the northernmost limit for rice paddy cultivation. Breed Sci. (2017) 67:191-206. doi: 10.1270/jsbbs.16155

32. Descalsota-Empleo GI, Amparado A, Inabangan-Asilo MA, Tesoro F, Stangoulis J, Reinke R, et al. Genetic mapping of QTL for agronomic traits and grain mineral elements in rice. Crop J. (2019) 7:56072. doi: 10.1016/j.cj.2019.03.002

33. Chattopadhyay K, Behera L, Bagchi TB, Sardar SS, Moharana N, Patra NR, et al. Detection of stable QTLs for grain protein content in rice (Oryza sativa L.) employing high throughput phenotyping and genotyping platforms. Sci Rep. (2019) 9:3196. doi: 10.1038/s41598-019-39863-2

34. Jeong OY, Lee JH, Jeong EG, Chun A, Bombay M, Banzon Ancheta M, et al. Analysis of QTL responsible for grain iron and zinc content in doubled haploid lines of rice (Oryza sativa) derived from an intra-japonica cross. Plant Breed. (2020) 139:344-55. doi: 10.1111/pbr.12787

35. Swamy B, Kaladhar K, Anuradha K, Batchu AK, Longvah T, Sarla N. QTL analysis for grain iron and zinc concentrations in two O. nivara derived backcross populations. Rice Sci. (2018) 25:197207. doi: 10.1016/j.rsci.2018.06.003

36. Descalsota GIL, Swamy B, Zaw H, Inabangan-Asilo MA, Amparado A, Mauleon R, et al. Genome-wide association mapping in a rice MAGIC Plus population detects QTLs and genes useful for biofortification. Front Plant Sci. (2018) 9:1347. doi: 10.3389/fpls.2018.01347

37. Xu Q, Zheng T-Q, Hu X, Cheng L-R, Xu J-L, Shi Y-M, et al. Examining two sets of introgression lines in rice (Oryza sativa L.) reveals favorable alleles that improve grain $\mathrm{Zn}$ and Fe concentrations. PLoS ONE. (2015) 10:e0131846. doi: 10.1371/journal.pone.0131846

38. Agarwal S, VGN TV, Kotla A, Mangrauthia SK, Neelamraju S. Expression patterns of QTL based and other candidate genes in Madhukar× Swarna RILs with contrasting levels of iron and zinc in unpolished rice grains. Gene. (2014) 546:430-6. doi: 10.1016/j.gene.2014.05.069

39. Yun BW, Kim MG, Handoyo T, Kim KM. Analysis of rice grain qualityassociated quantitative trait loci by using genetic mapping. Am J Plant Sci. (2014) 5:1125-32. doi: 10.4236/ajps.2014.59125

40. Lee G-H, Yun B-W, Kim K-M. Analysis of QTLs associated with the rice quality related gene by double haploid populations. Int J Genomics. (2014) 2014:781832. doi: 10.1155/2014/781832

41. Zhang M, Pinson SR, Tarpley L, Huang X-Y, Lahner B, Yakubova E, et al. Mapping and validation of quantitative trait loci associated with concentrations of 16 elements in unmilled rice grain. Theor Appl Genet. (2014) 127:137-65. doi: 10.1007/s00122-013-2207-5

42. Gande NK, Kundur PJ, Soman R, Ambati R, Ashwathanarayana $\mathrm{R}$, Bekele $\mathrm{BD}$, et al. Identification of putative candidate gene markers for grain zinc content using recombinant inbred lines (RIL) population of IRRI38 X Jeerigesanna. Afr J Biotechnol. (2014) 13:657-63. doi: 10.5897/AJB2013.13334

43. Peng B, Wang L, Fan C, Jiang G, Luo L, Li Y, et al. Comparative mapping of chalkiness components in rice using five populations across two environments. BMC Genet. (2014) 15:49. doi: 10.1186/1471-2156-15-49

44. Gu R, Chen F, Liu B, Wang X, Liu J, Li P, et al. Comprehensive phenotypic analysis and quantitative trait locus identification for grain mineral concentration, content, and yield in maize (Zea mays L.). Theor Appl Genet. (2015) 128:1777-89. doi: 10.1007/s00122-015-2546-5

45. Baxter IR, Gustin JL, Settles AM, Hoekenga OA. Ionomic characterization of maize kernels in the intermated B73 $\times$ Mo17 population. Crop Sci. (2013) 53:208-20. doi: 10.2135/cropsci2012.02.0135

46. Kumar S, Hash CT, Nepolean T, Mahendrakar MD, Satyavathi CT, Singh $\mathrm{G}$, et al. Mapping grain iron and zinc content quantitative trait loci in an iniadi-derived immortal population of pearl millet. Genes. (2018) 9:248. doi: 10.3390/genes 9050248
47. Anuradha N, Satyavathi CT, Bharadwaj C, Nepolean T, Sankar SM, Singh SP, et al. Deciphering genomic regions for high grain iron and zinc content using association mapping in pearl millet. Front Plant Sci. (2017) 8:412. doi: 10.3389/fpls.2017.00412

48. Izquierdo P, Astudillo C, Blair MW, Iqbal AM, Raatz B, Cichy KA. Meta-QTL analysis of seed iron and zinc concentration and content in common bean (Phaseolus vulgaris L.). Theor Appl Genet. (2018) 131:164558. doi: 10.1007/s00122-018-3104-8

49. Blair MW, Izquierdo P, Astudillo C, Grusak MA. A legume biofortification quandary: variability and genetic control of seed coat micronutrient accumulation in common beans. Front Plant Sci. (2013) 4:275. doi: 10.3389/fpls.2013.00275

50. Casanas F, Pérez-Vega E, Almirall A, Plans M, Sabaté J, Ferreira JJ. Mapping of QTL associated with seed chemical content in a RIL population of common bean (Phaseolus vulgaris L.). Euphytica. (2013) 192:27988. doi: 10.1007/s10681-013-0880-8

51. Blair MW, Izquierdo P. Use of the advanced backcross-QTL method to transfer seed mineral accumulation nutrition traits from wild to Andean cultivated common beans. Theor Appl Genet. (2012) 125:101531. doi: 10.1007/s00122-012-1891-x

52. Blair MW, Astudillo C, Rengifo J, Beebe SE, Graham R. QTL analyses for seed iron and zinc concentrations in an intra-genepool population of Andean common beans (Phaseolus vulgaris L.). Theor Appl Genet. (2011) 122:511-21. doi: 10.1007/s00122-010-1465-8

53. Ates D, Aldemir S, Yagmur B, Kahraman A, Ozkan H, Vandenberg A, et al. QTL mapping of genome regions controlling manganese uptake in lentil seed. G3. (2018) 8:1409-16. doi: 10.1534/g3.118.200259

54. Aldemir S, Ateş D, Temel HY, Yagmur B, Alsaleh A, Kahriman A, et al. QTLs for iron concentration in seeds of the cultivated lentil (Lens culinaris Medic.) via genotyping by sequencing. Turkish J Agric. Forest. (2017) 41:24355. doi: 10.3906/tar-1610-33

55. Kundu SS. Zinc Biofortification of Lentil. University of Saskatchewan (2016).

56. Ates D, Sever T, Aldemir S, Yagmur B, Temel HY, Kaya HB, et al. Identification QTLs controlling genes for Se uptake in lentil seeds. PLOS ONE. (2016) 11:e0149210. doi: 10.1371/journal.pone. 0149210

57. Aldemir S, Sever T, Ates D, Yagmur B, Kaya H, Temel H, et al. QTL mapping of genes controlling Fe uptake in lentil (Lens culinaris L.) seed using recombinant inbred lines. In: Plant and Animal Genome Conference (San Diego, CA) (2014).

58. Ates D, Aldemir S, Sever T, Yagmur B, Kaya H, Temel H, et al. "Identification of QTLs controlling genes to Mn and Zn uptake in lentil seeds," in Plant and Animal Genome XXII, San Diego, CA (2014). p. 11-5.

59. Ma Y, Coyne CJ, Grusak MA, Mazourek M, Cheng P, Main D, et al. Genome-wide SNP identification, linkage map construction and QTL mapping for seed mineral concentrations and contents in pea (Pisum sativum L.). BMC Plant Biol. (2017) 17:43. doi: 10.1186/s12870-0160956-4

60. Bangar P. Effect of Iron and Carotenoid Concentration on Iron Bioavailability in Pea Pisum sativum L. Saskatoon, SK: University of Saskatchewan (2016).

61. Shunmugam A, Liu X, Stonehouse R, Tar'An B, Bett K, Sharpe A, et al. Mapping seed phytic acid concentration and iron bioavailability in a pea recombinant inbred line population. Crop Sci. (2015) 55:82836. doi: 10.2135/cropsci2014.08.0544

62. Upadhyaya HD, Bajaj D, Das S, Kumar V, Gowda C, Sharma S, et al. Genetic dissection of seed-iron and zinc concentrations in chickpea. Sci Rep. (2016) 6:24050. doi: 10.1038/srep24050

63. Jadhav A, Rayate S, Mhase L, Thudi M, Chitikineni A, Harer P, et al. Markertrait association study for protein content in chickpea (Cicer arietinum L.). J Genet. (2015) 94:279-86. doi: 10.1007/s12041-015-0529-6

64. Diapari M, Sindhu A, Bett K, Deokar A, Warkentin TD, Tar'an B. Genetic diversity and association mapping of iron and zinc concentrations in chickpea (Cicer arietinum L.). Genome. (2014) 57:459-68. doi: 10.1139/gen-2014-0108

65. Huang L, He H, Chen W, Ren X, Chen Y, Zhou X, et al. Quantitative trait locus analysis of agronomic and quality-related traits in cultivated peanut (Arachis hypogaea L.). Theor Appl Genet. (2015) 128:110315. doi: 10.1007/s00122-015-2493-1 
66. Wang ML, Khera P, Pandey MK, Wang H, Qiao L, Feng S, et al. Genetic mapping of QTLs controlling fatty acids provided insights into the genetic control of fatty acid synthesis pathway in peanut (Arachis hypogaea L.). PLoS ONE. (2015) 10:e0119454. doi: 10.1371/journal.pone.0119454

67. Pandey MK, Wang ML, Qiao L, Feng S, Khera P, Wang H, et al. Identification of QTLs associated with oil content and mapping FAD2 genes and their relative contribution to oil quality in peanut (Arachis hypogaea L.). BMC Genet. (2014) 15:133. doi: 10.1186/s12863-014-0133-4

68. Vijayata S. Genotypic Response and QTL Identification for Micronutrient (Iron and Zinc) Contents in Mungbean [Vigna radiata (L.) Wilczek]. CCSHAU. (2013).

69. Sompong U, Somta P, Raboy V, Srinives P. Mapping of quantitative trait loci for phytic acid and phosphorus contents in seed and seedling of mungbean (Vigna radiata (L.) Wilczek). Breeding Sci. (2012) 62:8792. doi: $10.1270 /$ jsbbs. 62.87

70. Ma Y, Ma W, Hu D, Zhang X, Yuan W, He X, et al. QTL mapping for protein and sulfur-containing amino acid contents using a high-density bin-map in soybean (Glycine max L. Merr.). J Agric Food Chem. (2019) 67:12313-21. doi: 10.1021/acs.jafc.9b04497

71. Akond M, Yuan J, Liu S, Kantartzi SK, Meksem K, Bellaloui $\mathrm{N}$, et al. Detection of QTL underlying seed quality components in soybean [Glycine $\max$ (L.) Merr.]. Can J Plant Sci. (2018) 98:881-8. doi: 10.1139/cjps-2017-0204

72. Cai Z, Cheng Y, Ma Z, Liu X, Ma Q, Xia Q, et al. Fine-mapping of QTLs for individual and total isoflavone content in soybean (Glycine max L.) using a high-density genetic map. Theor Appl Genet. (2018) 131:55568. doi: 10.1007/s00122-017-3018-x

73. Warrington C, Abdel-Haleem H, Hyten D, Cregan P, Orf J, Killam A, et al. QTL for seed protein and amino acids in the Benning $\times$ Danbaekkong soybean population. Theor Appl Genet. (2015) 128:83950. doi: 10.1007/s00122-015-2474-4

74. Zeng A, Chen P, Shi A, Wang D, Zhang B, Orazaly M, et al. Identification of quantitative trait loci for sucrose content in soybean seed. Crop Sci. (2014) 54:554-64. doi: 10.2135/cropsci2013.01.0036

75. Wang J, Chen P, Wang D, Shannon G, Zeng A, Orazaly M, et al. Identification and mapping of stable QTL for protein content in soybean seeds. Mol Breed. (2015) 35:92. doi: 10.1007/s11032-015-0285-6

76. Wang J, Chen P, Wang D, Shannon G, Shi A, Zeng A, et al. Identification of quantitative trait loci for oil content in soybean seed. Crop Sci. (2015) 55:23-34. doi: 10.2135/cropsci2014.04.0280

77. Ning L, Sun P, Wang Q, Ma D, Hu Z, Zhang D, et al. Genetic architecture of biofortification traits in soybean (Glycine max L. Merr.) revealed through association analysis and linkage mapping. Euphytica. (2015) 204:35369. doi: 10.1007/s10681-014-1340-9

78. Wang X, Jiang G-L, Green M, Scott RA, Song Q, Hyten DL, et al. Identification and validation of quantitative trait loci for seed yield, oil and protein contents in two recombinant inbred line populations of soybean. Mol Genet Genomics. (2014) 289:935-49. doi: 10.1007/s00438-014-0865-X

79. Yan L, Xing L-L, Yang C-Y, Chang R-Z, Zhang M-C, Qiu L-J. Identification of quantitative trait loci associated with soybean seed protein content using two populations derived from crosses between glycine max and glycine soja. Plant Genet Resour. (2014) 12:S104. doi: 10.1017/S1479262114000379

80. Ramamurthy RK, Jedlicka J, Graef GL, Waters BM. Identification of new QTLs for seed mineral, cysteine, and methionine concentrations in soybean [Glycine max (L.) Merr.]. Mol Breed. (2014) 34:43145. doi: 10.1007/s11032-014-0045-Z

81. Li B, Tian L, Zhang J, Huang L, Han F, Yan S, et al. Construction of a high-density genetic map based on large-scale markers developed by specific length amplified fragment sequencing (SLAF-seq) and its application to QTL analysis for isoflavone content in Glycine max. BMC Genomics. (2014) 15:1086. doi: 10.1186/1471-2164-15-1086

82. Orazaly $M$, Chen $\mathrm{P}$, Zhang $\mathrm{B}$, Zeng A. Quantitative trait loci mapping for seed calcium content of soybean. Crop Sci. (2014) 54:500-6. doi: 10.2135/cropsci2013.01.0026

83. Akond M, Liu S, Boney M, Kantarrtzi SK, Meksem K, Bellaloui N, et al. Identification of quantitative trait loci (QTL) underlying protein, oil, and five major fatty acids' contents in soybean. Am J Plant Sci. (2014) 5:158-67. doi: 10.4236/ajps.2014.51021
84. Qi Z, Hou M, Han X, Liu C, Jiang H, Xin D, et al. Identification of quantitative trait loci (QTL s) for seed protein concentration in soybean and analysis for additive effects and epistatic effects of QTL s under multiple environments. Plant Breed. (2014) 133:499-507. doi: 10.1111/pbr.12179

85. Behnke N, Suprianto E, Möllers C. A major QTL on chromosome C05 significantly reduces acid detergent lignin (ADL) content and increases seed oil and protein content in oilseed rape (Brassica napus L.). Theor Appl Genet. (2018) 131:2477-92. doi: 10.1007/s00122-018-3167-6

86. Gacek K, Bayer PE, Bartkowiak-Broda I, Szala L, Bocianowski J, Edwards D, et al. Genome-wide association study of genetic control of seed fatty acid biosynthesis in Brassica napus. Front Plant Sci. (2017) 7:2062. doi: 10.3389/fpls.2016.02062

87. Gu J, Chao H, Wang H, Li Y, Li D, Xiang J, et al. Identification of the relationship between oil body morphology and oil content by microstructure comparison combining with QTL analysis in Brassica napus. Front Plant Sci. (2017) 7:1989. doi: 10.3389/fpls.2016.01989

88. Basnet RK, Del Carpio DP, Xiao D, Bucher J, Jin M, Boyle K, et al. A systems genetics approach identifies gene regulatory networks associated with fatty acid composition in Brassica rapa seed. Plant Physiol. (2016) 170:568-85. doi: 10.1104/pp.15.00853

89. Javed N, Geng J, Tahir M, McVetty P, Li G, Duncan RW. Identification of QTL influencing seed oil content, fatty acid profile and days to flowering in Brassica napus L. Euphytica. (2016) 207:191-211. doi: 10.1007/s10681-015-1565-2

90. Zou J, Zhao Y, Liu P, Shi L, Wang X, Wang M, et al. Seed quality traits can be predicted with high accuracy in Brassica napus using genomic data. PLoS ONE. (2016) 11:e0166624. doi: 10.1371/journal.pone.0166624

91. Jiang C, Shi J, Li R, Long Y, Wang H, Li D, et al. Quantitative trait loci that control the oil content variation of rapeseed (Brassica napus L.). Theor Appl Genet. (2014) 127:957-68. doi: 10.1007/s00122-014-2271-5

92. Körber N, Bus A, Li J, Parkin IA, Wittkop B, Snowdon RJ, et al. Agronomic and seed quality traits dissected by genome-wide association mapping in Brassica napus. Front Plant Sci. (2016) 7:386. doi: 10.3389/fpls.2016.00386

93. Xu J, Long $\mathrm{Y}, \mathrm{Wu} \mathrm{J}, \mathrm{Xu} \mathrm{H}$, Wen J, Meng J, et al. QTL mapping and analysis of the embryo and maternal plant for three limiting amino acids in rapeseed meal. Eur Food Res Technol. (2015) 240:14758. doi: 10.1007/s00217-014-2316-7

94. Huang X-Q, Huang T, Hou G-Z, Li L, Hou Y, Lu Y-H. Identification of QTLs for seed quality traits in rapeseed (Brassica napus L.) using recombinant inbred lines (RILs). Euphytica. (2016) 210:1-16. doi: 10.1007/s10681-016-1675-5

95. Wang X, Long Y, Yin Y, Zhang C, Gan L, Liu L, et al. New insights into the genetic networks affecting seed fatty acid concentrations in Brassica napus. BMC Plant Biol. (2015) 15:91. doi: 10.1186/s12870-015-0475-8

96. Wen J, Xu J, Long Y, Xu H, Wu J, Meng J, et al. Mapping QTLs controlling beneficial fatty acids based on the embryo and maternal plant genomes in Brassica napus L. J Am Oil Chem Soc. (2015) 92:54152. doi: 10.1007/s11746-015-2618-3

97. Lemesh V, Mozgova G, Grushetskaya Z, Sidorenko E, Piluk YE, Bakanovskaya A. The use of specific DNA markers for the identification of alleles of the FAD3 genes in rape (Brassica napus L.). Russ J Genet. (2015) 51:765-73. doi: 10.1134/S1022795415080049

98. Liu F, Wang G, Liu R, Guan C. The promoter of fatty acid desaturase on chromosome C5 in Brassica napus drives high-level expression in seeds. Plant Biotechnol Rep. (2016) 10:369-81. doi: 10.1007/s11816-0160407-6

99. Yu B, Boyle K, Zhang W, Robinson SJ, Higgins E, Ehman L, et al. Multitrait and multi-environment QTL analysis reveals the impact of seed colour on seed composition traits in Brassica napus. Mol Breed. (2016) 36:111. doi: 10.1007/s11032-016-0521-8

100. Wen J, Xu JF, Long Y, Wu JG, Xu HM, Meng JL, et al. QTL mapping based on the embryo and maternal genetic systems for non-essential amino acids in rapeseed (Brassica napus L.) meal. J Sci Food Agric. (2016) 96:46573. doi: $10.1002 /$ jsfa.7112

101. Wells R, Trick M, Soumpourou E, Clissold L, Morgan C, Werner $\mathrm{P}$, et al. The control of seed oil polyunsaturate content in the polyploid crop species Brassica napus. Mol Breed. (2014) 33:34962. doi: $10.1007 / \mathrm{s} 11032-013-9954-5$ 
102. Zhang W, Hu D, Raman R, Guo S, Wei Z, Shen X, et al. Investigation of the genetic diversity and quantitative trait loci accounting for important agronomic and seed quality traits in Brassica carinata. Front Plant Sci. (2017) 8:615. doi: 10.3389/fpls.2017.00615

103. Gupta S, Sangha M, Kaur G, Banga S, Gupta M, Kumar H, et al. QTL analysis for phytonutrient compounds and the antioxidant molecule in mustard (Brassica juncea L.). Euphytica. (2015) 201:345-56. doi: 10.1007/s10681-014-1204-3

104. Saini N, Singh N, Kumar A, Vihan N, Yadav S, Vasudev S. Development and validation of functional CAPS markers for the FAE genes in Brassica juncea and their use in marker-assisted selection. Breed Sci. (2016) 66:8317. doi: $10.1270 /$ jsbbs. 16132

105. Karim MM, Tonu NN, Hossain MS, Funaki T, Meah MB, Hossain DM, et al. Marker-assisted selection of low erucic acid quantity in short duration Brassica rapa. Euphytica. (2016) 208:535-44. doi: 10.1007/s10681-015-1596-8

106. Singer SD, Weselake RJ, Rahman H. Development and characterization of low $\alpha$-linolenic acid Brassica oleracea lines bearing a novel mutation in a 'class a'FATTY ACID DESATURASE 3gene. BMC Genet. (2014) 15:94. doi: 10.1186/s12863-014-0094-7

107. Cakmak I, Pfeiffer WH, McClafferty B. Biofortification of durum wheat with zinc and iron. Cereal Chem. (2010) 87:10-20. doi: 10.1094/CCHEM-87-1-0010

108. Singh U, Praharaj C, Singh SS, Singh NP. Biofortification of Food Crops. New Delhi: Springer (2016).

109. Khazaei H, Podder R, Caron CT, Kundu SS, Diapari M, Vandenberg A, et al. Marker-trait association analysis of iron and zinc concentration in lentil (Lens culinaris Medik.) seeds. Plant Genome. (2017) 10:18. doi: 10.3835/plantgenome2017.02.0007

110. Kumar J, Gupta DS, Kumar S, Gupta S, Singh NP. Current knowledge on genetic biofortification in lentil. J Agric Food Chem. (2016) 64:638396. doi: 10.1021/acs.jafc.6b02171

111. Singh A, Sharma V, Dikshit H, Singh D, Aski M, Prakash P, et al. Microsatellite marker-based genetic diversity analysis of elite lentil lines differing in grain iron and zinc concentration. J Plant Biochem Biotechnol. (2017) 26:199-207. doi: 10.1007/s13562-016-0382-6

112. Saini N, Koramutla MK, Singh N, Singh S, Singh R, Yadav S, et al. Promoter polymorphism in FAE1. 1 and FAE1. 2 genes associated with erucic acid content in Brassica juncea. Mol Breed. (2019) 39:75. doi: 10.1007/s11032-019-0971-x

113. Gunstone FD, Harwood JL, Dijkstra AJ. The Lipid Handbook with CD-ROM. Buca Raton, FL: CRC Press (2007)

114. Norden A, Gorbet D, Knauft D, Young C. Variability in oil quality among peanut genotypes in the Florida breeding program. Peanut Sci. (1987) 14:7-11. doi: 10.3146/i0095-3679-14-1-3

115. Moore K, Knauft D. The inheritance of high oleic acid in peanut. J Hered. (1989) 80:252-3. doi: 10.1093/oxfordjournals.jhered.a110845

116. Hu X, Zhang S, Miao H, Cui F, Shen Y, Yang W, et al. High-density genetic map construction and identification of QTLs controlling oleic and linoleic acid in peanut using SLAF-seq and SSRs. Sci Rep. (2018) 8:5479. doi: 10.1038/s41598-018-23873-7

117. Rehman HM, Cooper JW, Lam HM, Yang SH. Legume biofortification is an underexploited strategy for combatting hidden hunger. Plant Cell Environ. (2019) 42:52-70. doi: 10.1111/pce.13368

118. Nöthlings U, Murphy SP, Wilkens LR, Henderson BE, Kolonel LN. Dietary glycemic load, added sugars, and carbohydrates as risk factors for pancreatic cancer: the multiethnic cohort study. Am J Clin Nutr. (2007) 86:1495501. doi: 10.1093/ajcn/86.5.1495

119. Prasanna BM, Palacios-Rojas N, Hossain F, Muthusamy V, Menkir A, Dhliwayo T, et al. Molecular breeding for nutritionally enriched maize: status and prospects. Front Genet. (2020) 10:1392. doi: 10.3389/fgene.2019.01392

120. Guzman C, Peña RJ, Singh R, Autrique E, Dreisigacker S, Crossa J, et al. Wheat quality improvement at CIMMYT and the use of genomic selection on it. Appl Transl Genomics. (2016) 11:3-8. doi: 10.1016/j.atg.2016. 10.004

121. Poland JA, Rife TW. Genotyping-by-sequencing for plant breeding and genetics. Plant Genome. (2012) 5:92102. doi: $10.3835 /$ plantgenome2012.05.0005
122. Heffner EL, Jannink JL, Sorrells ME. Genomic selection accuracy using multifamily prediction models in a wheat breeding program. Plant Genome. (2011) 4:65-75. doi: 10.3835/plantgenome2010.12.0029

123. Lorenz AJ, Chao S, Asoro FG, Heffner EL, Hayashi T, Iwata H, et al. Genomic selection in plant breeding: knowledge and prospects. Adv Agron. (2011) 110:77-123. doi: 10.1016/B978-0-12-385531-2.00002-5

124. Hossain F, Muthusamy V, Pandey N, Vishwakarma AK, Baveja A, Zunjare $\mathrm{RU}$, et al. Marker-assisted introgression of opaque2 allele for rapid conversion of elite hybrids into quality protein maize. J Genet. (2018) 97:287-98. doi: 10.1007/s12041-018-0914-Z

125. Kumar J, Jaiswal V, Kumar A, Kumar N, Mir R, Kumar S, et al. Introgression of a major gene for high grain protein content in some Indian bread wheat cultivars. Field Crops Res. (2011) 123:226-33. doi: 10.1016/j.fcr.2011. 05.013

126. Tyagi S, Mir R, Kaur H, Chhuneja $\mathrm{P}$, Ramesh B, Balyan H, et al. Marker-assisted pyramiding of eight QTLs/genes for seven different traits in common wheat (Triticum aestivum L.). Mol Breed. (2014) 34:16775. doi: 10.1007/s11032-014-0027-1

127. Vishwakarma MK, Arun B, Mishra V, Yadav P, Kumar H, Joshi AK. Marker-assisted improvement of grain protein content and grain weight in Indian bread wheat. Euphytica. (2016) 208:313-21. doi: 10.1007/s10681-0151598-6

128. Vishwakarma MK, Mishra V, Gupta P, Yadav P, Kumar H, Joshi AK. Introgression of the high grain protein gene $\mathrm{Gpc}-\mathrm{B} 1$ in an elite wheat variety of Indo-Gangetic plains through marker assisted backcross breeding. Curr Plant Biol. (2014) 1:60-7. doi: 10.1016/j.cpb.2014.09.003

129. Randhawa HS, Asif M, Pozniak C, Clarke JM, Graf RJ, Fox SL, et al. Application of molecular markers to wheat breeding in Canada. Plant Breed. (2013) 132:458-71. doi: 10.1111/pbr.12057

130. Wheat Cap Coordinated Agricultural Project. University of California. Available online at: https://www.triticeaecap.org/t-cap-variety-andgermplasm-releases_2011_2013/(accessed July 20, 2020)

131. Lau WCP, Latif MA. Current breeding approaches for developing rice with improved grain and nutritional qualities. In: A. M. Iqbal Qureshi, Z. A. Dar, and S. H. Wani, eds. Quality Breeding in Field Crops. Srinagar: Springer (2019). p. 199-216.

132. Amarawathi Y, Singh R, Singh AK, Singh VP, Mohapatra T, Sharma TR, et al. Mapping of quantitative trait loci for basmati quality traits in rice (Oryza sativa L.). Mol Breed. (2008) 21:49-65. doi: 10.1007/s11032-007-9108-8

133. Lau WCP, Rafii MY, Ismail MR, Puteh A, Latif MA, Asfaliza R, et al. Development of advanced fragrant rice lines from MR269× Basmati 370 through marker-assisted backcrossing. Euphytica. (2017) 213:11. doi: 10.1007/s10681-016-1794-z

134. Prado JR, Segers G, Voelker T, Carson D, Dobert R, Phillips J, et al. Genetically engineered crops: from idea to product. Annu Rev Plant Biol. (2014) 65:769-90. doi: 10.1146/annurev-arplant-050213-040039

135. Gao C. Genome editing in crops: from bench to field. Natl Sci Rev. (2015) 2:13-5. doi: 10.1093/nsr/nwu054

136. Kim Y-G, Cha J, Chandrasegaran S. Hybrid restriction enzymes: zinc finger fusions to Fok I cleavage domain. Proc Natl Acad Sci USA. (1996) 93:115660. doi: 10.1073/pnas.93.3.1156

137. Christian M, Cermak T, Doyle EL, Schmidt C, Zhang F, Hummel A, et al. Targeting DNA double-strand breaks with TAL effector nucleases. Genetics. (2010) 186:757-61. doi: 10.1534/genetics.110.120717

138. Jinek M, Chylinski K, Fonfara I, Hauer M, Doudna JA, Charpentier E. A programmable dual-RNA-guided DNA endonuclease in adaptive bacterial immunity. Science. (2012) 337:816-21. doi: 10.1126/science.1225829

139. Symington LS, Gautier J. Double-strand break end resection and repair pathway choice. Annu Rev Genet. (2011) 45:24771. doi: 10.1146/annurev-genet-110410-132435

140. Voytas DF, Gao C. Precision genome engineering and agriculture: opportunities and regulatory challenges. PLoS Biol. (2014) 12:e1001877. doi: 10.1371/journal.pbio.1001877

141. Sánchez-León S, Gil-Humanes J, Ozuna CV, Giménez MJ, Sousa C, Voytas DF, et al. Low-gluten, nontransgenic wheat engineered with CRISPR/Cas9. Plant Biotechnol J. (2018) 16:902-10. doi: 10.1111/pbi.12837

142. Jouanin A, Schaart JG, Boyd LA, Cockram J, Leigh FJ, Bates R, et al. Outlook for coeliac disease patients: towards bread wheat with hypoimmunogenic 
gluten by gene editing of $\alpha$-and $\gamma$-gliadin gene families. BMC Plant Biol. (2019) 19:333. doi: 10.1186/s12870-019-1889-5

143. Sun Y, Jiao G, Liu Z, Zhang X, Li J, Guo X, et al. Generation of high-amylose rice through CRISPR/Cas9-mediated targeted mutagenesis of starch branching enzymes. Front Plant Sci. (2017) 8:298. doi: 10.3389/fpls.2017.00298

144. Shan $\mathrm{Q}$, Zhang $\mathrm{Y}$, Chen $\mathrm{K}$, Zhang $\mathrm{K}$, Gao C. Creation of fragrant rice by targeted knockout of the Os BADH 2 gene using TALEN technology. Plant Biotechnol J. (2015) 13:791-800. doi: 10.1111/pbi.12312

145. Abe K, Araki E, Suzuki Y, Toki S, Saika H. Production of high oleic/low linoleic rice by genome editing. Plant Physiol Biochem. (2018) 131:5862. doi: 10.1016/j.plaphy.2018.04.033

146. Endo A, Saika H, Takemura M, Misawa N, Toki S. A novel approach to carotenoid accumulation in rice callus by mimicking the cauliflower Orange mutation via genome editing. Rice. (2019) 12:81. doi: 10.1186/s12284-019-0345-3

147. Zhu Y, Lin Y, Chen S, Liu H, Chen Z, Fan M, et al. CRISPR/Cas9-mediated functional recovery of the recessive rc allele to develop red rice. Plant Biotechnol J. (2019) 17:2096-105. doi: 10.1111/pbi.13125

148. Pioneer. DuPont Pioneer's Next Generation of Waxy Corn Shows the Green Side of CRISPR-Cas9. Feburary 16, 2017. Available online at: https://biovox. eu/dupont-pioneer-rsquo-s-next-generation- of-waxy-corn-shows-the-gr/ (accessed July 16, 2020).

149. Haun W, Coffman A, Clasen BM, Demorest ZL, Lowy A, Ray E, et al. Improved soybean oil quality by targeted mutagenesis of the fatty acid desaturase 2 gene family. Plant Biotechnol J. (2014) 12:93440. doi: $10.1111 /$ pbi.12201

150. Demorest ZL, Coffman A, Baltes NJ, Stoddard TJ, Clasen BM, Luo S, et al. Direct stacking of sequence-specific nuclease-induced mutations to produce high oleic and low linolenic soybean oil. BMC Plant Biol. (2016) 16:225. doi: 10.1186/s12870-016-0906-1

151. Al Amin N, Ahmad N, Wu N, Pu X, Ma T, Du Y, et al. CRISPRCas9 mediated targeted disruption of FAD2-2 microsomal omega6 desaturase in soybean (Glycine max. L). BMC Biotechnol. (2019) 19:9. doi: 10.1186/s12896-019-0501-2

152. Du H, Zeng X, Zhao M, Cui X, Wang Q, Yang H, et al. Efficient targeted mutagenesis in soybean by TALENs and CRISPR/Cas9. J Biotechnol. (2016) 217:90-7. doi: 10.1016/j.jbiotec.2015.11.005

153. Yuan M, Zhu J, Gong L, He L, Lee C, Han S, et al. Mutagenesis of FAD2 genes in peanut with CRISPR/Cas9 based gene editing. BMC Biotechnol. (2019) 19:24. doi: 10.1186/s12896-019-0516-8

154. Andersson M, Turesson H, Nicolia A, Fält A-S, Samuelsson M, Hofvander P. Efficient targeted multiallelic mutagenesis in tetraploid potato (Solanum tuberosum) by transient CRISPR-Cas9 expression in protoplasts. Plant Cell Rep. (2017) 36:117-28. doi: 10.1007/s00299-016-2062-3

155. Clasen BM, Stoddard TJ, Luo S, Demorest ZL, Li J, Cedrone F, et al. Improving cold storage and processing traits in potato through targeted gene knockout. Plant Biotechnol J. (2016) 14:169-76. doi: 10.1111/pbi.12370

156. Okuzaki A, Ogawa T, Koizuka C, Kaneko K, Inaba M, Imamura J, et al. CRISPR/Cas9-mediated genome editing of the fatty acid desaturase 2 gene in Brassica napus. Plant Physiol Biochem. (2018) 131:639. doi: 10.1016/j.plaphy.2018.04.025

157. Huang H, Cui T, Zhang L, Yang Q, Yang Y, Xie K, et al. Modifications of fatty acid profile through targeted mutation at BnaFAD2 gene with CRISPR/Cas9-mediated gene editing in Brassica napus. Theor Appl Genet. (2020) 133:2401-11. doi: 10.1007/s00122-020-03607-y

158. Chen L, Magliano DJ, Zimmet PZ. The worldwide epidemiology of type 2 diabetes mellitus-present and future perspectives. Nat Rev Endocrinol. (2012) 8:228-36. doi: 10.1038/nrendo.2011.183

159. Qiu Y, Liu Q, Beta T. Antioxidant properties of commercial wild rice and analysis of soluble and insoluble phenolic acids. Food Chem. (2010) 121:140-7. doi: 10.1016/j.foodchem.2009.12.021

160. Furukawa T, Maekawa M, Oki T, Suda I, Iida S, Shimada H, et al. The Rc and Rd genes are involved in proanthocyanidin synthesis in rice pericarp. Plant J. (2007) 49:91-102. doi: 10.1111/j.1365-313X.2006.02958.x

161. Sweeney MT, Thomson MJ, Pfeil BE, McCouch S. Caught red-handed: Rc encodes a basic helix-loop-helix protein conditioning red pericarp in rice. Plant Cell. (2006) 18:283-94. doi: 10.1105/tpc.105.038430
162. Jiang WZ, Henry IM, Lynagh PG, Comai L, Cahoon EB, Weeks DP. Significant enhancement of fatty acid composition in seeds of the allohexaploid, Camelina sativa, using CRISPR/Cas9 gene editing. Plant Biotechnol J. (2017) 15:648-57. doi: 10.1111/pbi.12663

163. Morineau C, Bellec Y, Tellier F, Gissot L, Kelemen Z, Nogué F, et al. Selective gene dosage by CRISPR-Cas9 genome editing in hexaploid Camelina sativa. Plant Biotechnol J. (2017) 15:729-39. doi: 10.1111/pbi.12671

164. Ozseyhan ME, Kang J, Mu X, Lu C. Mutagenesis of the FAE1 genes significantly changes fatty acid composition in seeds of Camelina sativa. Plant Physiol Biochem. (2018) 123:1-7. doi: 10.1016/j.plaphy.2017. 11.021

165. Chatrath R, V Tiwari, V Gupta, S Kumar, SK Singh, CN Mishra, et al. WB 2: a high yielding bread wheat variety for irrigated timely sown conditions of North Western plains zone of India. Wheat Barley Res. (2018) 10:404. doi: 10.25174/2249-4065/2018/81917

166. Singh R, Govindan V, Andersson MS. Zinc-biofortified wheat: harnessing genetic diversity for improved nutritional quality. Science Brief: Biofortification No. 1 (May 2017). CIMMYT, HarvestPlus, and the Global Crop Diversity Trust. Bonn, Germany. (2017).

167. Gupta A, Singh C, Kumar V, Tyagi B, Tiwari V, Chatrath R, et al. Wheat Varieties Notified in India Since 1965. ICAR-Indian Institute of Wheat \& Barley Research, Karnal (2018).

168. Velu G, Singh R, Balasubramaniam A, Mishra VK, Chand R, Tiwari C, et al. Reaching out to farmers with high zinc wheat varieties through public-private partnerships: an experience from eastern-gangetic plains of India. Adv Food Technol Nutr Sci. (2015) 3:73-5. doi: 10.17140/AFTNSO $\mathrm{J}-1-112$

169. Yadav R, Gaikwad KB, Singh GP, Kumar M, Singh PK, Sai Prasad SV, et al. HD 3226 Notification of crop varieties and registration of germplasm. Indian J Genet Plant Breed. (2019) 79:632-3.

170. Ravish C, Tiwari V, Singh G, Tiwari R, Tyagi BS. ICAR-Indian Institute of Wheat and Barley Research, Karnal, Haryana, India. ICAR-IIWBR Progress Report of AICRP on Wheat Barley 2017-18, Crop Improvement. (2018). p. 206.

171. Martinek P, Škorpík M, Chrpova J, Schweiger JFP. Development of the new winter wheat variety Skorpion with blue grain. Czech J Genet Plant Breed. (2013) 49:90-4. doi: 10.17221/7/2013-CJGPB

172. Hanková A, Matúšková K, Rückschloss, L, Žofajová A. Winter wheat Ps Karkulka. Agriculture. (2014) 60:159-61.

173. Garg M, Chawla M, Chunduri V, Kumar R, Sharma S, Sharma NK, et al. Transfer of grain colors to elite wheat cultivars and their characterization. J Cereal Sci. (2016) 71:138-44. doi: 10.1016/j.jcs.2016. 08.004

174. Eticha F, Grausgruber H, Siebenhandl-Ehn S, Berghofer E. Some agronomic and chemical traits of blue aleurone and purple pericarp wheat (Triticum L.). J Agric Sci Technol B. (2011) 1:48-58.

175. Bai Y, Li W, Sun S, Sun Y, Pei Z, Corke H. Evaluation on some quality characteristics of black-grained wheat 76. J Chin Cereal Oil Assoc. (2000) 15:6-9.

176. Gregorio GB, Senadhira D, Htut H, Graham RD. Breeding for trace mineral density in rice. Food Nutr Bull. (2000) 21:382-6. doi: 10.1177/156482650002100407

177. HarvestPlus. Bangladesh Releases New, Improved Zinc Rice Variety. (2015). Available online at: https://www.harvestplus.org/knowledge-market/inthe-news/bangladesh-releases-new-improved-zinc-rice-variety- 0 (accessed October 07, 2020).

178. Yadava D, Choudhury P, Hossain F, Kumar D. Biofortified Varieties: Sustainable way to Alleviate Malnutrition. New Delhi: Indian Council of Agricultural Research (2017).

179. Gupta HS, Hossain F, Muthusamy V. Biofortification of maize: an Indian perspective. Indian $J$ Genet. (2015) 75:122. doi: 10.5958/0975-6906.2015.00001.2

180. CIMMYT. Pakistan Releases First Quality Protein Maize Varieties. (2017). Available online at: https://www.cimmyt.org/news/pakistan-releases-firstquality-protein-maize-varieties/ (accessed July 10, 2020).

181. Vasal S. Quality protein maize development: an exciting experience. In: D. K. Friesen and A.F.E. Palmer eds. Integrated Approaches to Higher Maize Productivity in the New Millennium. Nairobi: CIMMYT and KARI (Kenya Agricultural Research Institute) (2002) 3-6. 
182. Sofi P, Wani SA, Rather A, Wani SH. Quality protein maize (QPM): genetic manipulation for the nutritional fortification of maize. J Plant Breed Crop Sci. (2009) 1:244-53. doi: 10.5897/JPBCS.9000113

183. Chaudhary D, Kumar S, Yadav O. Nutritive value of maize: improvements, applications and constraints. In: Maize: Nutrition Dynamics and Novel Uses. New Delhi: Springer. (2014). p. 3-17.

184. Zunjare RU, Hossain F, Muthusamy V, Baveja A, Chauhan HS, Bhat JS, et al. Development of biofortified maize hybrids through marker-assisted stacking of $\beta$-carotene hydroxylase, lycopene- $\varepsilon$-cyclase and opaque 2 genes. Front Plant Sci. (2018) 9:178. doi: 10.3389/fpls.2018.00178

185. Andersson MS, Saltzman A, Virk PS, Pfeiffer WH. Progress update: crop development of biofortified staple food crops under HarvestPlus. Afr J Food Agric Nutr Dev. (2017) 17:11905-35. doi: 10.18697/ajfand.78.HarvestPlus05

186. CIMMYT. CIMMYT-SARO Steps Up Efforts in War Against Malnutrition. (2015). Available online at: https://www.cimmyt.org/news/cimmyt-sarosteps-up-efforts-in-war-against-malnutrition/ (accessed July 10, 2020).

187. CIMMYT. Available online at: https://www.cimmyt.org/news/first-zincmaize-variety-launched-to-reduce-malnutrition-in-colombia/ (accessed July 10, 2020)

188. ICRISAT. Retrieved from: http://www.icrisat.org/africas-first-biofortifiedpearl-millet-variety-aims-to-combat-anaemia/ (accessed July 16, 2020).

189. ICRISAT. India Gets Its First Biofortified Sorghum. (2018). Retrieved from: https://www.icrisat.org/india-gets-its-first-biofortified-sorghum/ (accessed July 16, 2020)

190. ICRISAT. New Sorghum Varieties Released in Nigeria With Higher Iron, Yields and Drought Resilience. (2016). Available online at: https://www. icrisat.org/new-sorghum-varieties-will-fight-malnutrition-and-climatechange-in-sudan-and-sahel-region-of-nigeria/ (accessed July 10, 2020).

191. HarvestPlus. Uganda Releases Biofortified Beans to Address Iron Deficiency Anemia. (2016). Available online at: https://www.harvestplus.org/ knowledge-market/in-the-news/uganda-releases-biofortified-beansaddress-iron-deficiency-anemia (accessed July 10, 2020).

192. HarvestPlus. RWANDA Country Brief. Available online at: https://www. harvestplus.org/sites/default/files/HarvestPlus_CountryBrief_Rwanda.pdf (accessed July 10, 2020).

193. HarvestPlus. Democratic Republic of Congo Country Brief. Available online at: www.harvestplus.org

194. Mulambu J, Andersson M, Palenberg M, Pfeiffer W, Saltzman A, Birol E, et al. Iron beans in Rwanda: crop development and delivery experience. Afr J Food Agric Nutr Dev. (2017) 17:12026-50. doi: 10.18697/ajfand.78.HarvestPlus10

195. ICARDA. Food From Fallows: Capitalizing on Idle Land for Better Food Security in South Asia. Retrieved from: https://www.icarda.org/project/ alleviating-malnutrition-biofortified-lentil (accessed July 16, 2020).

196. Sarker A, Erskine W, Abu Bakr M, Matiur Rahman M, Ali Afzal M, Saxena MC. Lentil improvement in Bangladesh. A Success Story of Fruitful Partnership Between the Bangladesh Agricultural Research Institute and the International Center for Agricultural Research in the dry Areas. APAARI Publication (2004). p. 1-38.

197. ICARDA. 'BARIMasur-8': A New Micronutrient-Rich Lentil Variety Released in Bangladesh. Available online at: https://www.icarda.org/media/news/ barimasur-8-new-micronutrient-rich-lentil-variety-released-bangladesh (accessed July 11, 2020).

198. ICARDA. New Micronutrient Rich Lentil Varieties Released in Nepal and India. Available online at: https://www.icarda.org/media/news/newmicronutrient-rich-lentil-varieties-released-nepal-and-india (accessed July 11, 2020).

199. ICARDA. Pulses for Food Security, Nutrition and Environment: the Role of Science \& Technology to Enhance Productivity and Production of Pulses. (2015). Available online at: https://iyp2016.org/resources/documents/ related-documents/72-iyp-launch-dr-m-solh-presentation/file (accessed July 11, 2020).
200. Yadav DK, Hossain Firoz, Choudhary PR, Kumar Dinesh, Singh AK, Mohapatra T. Crop Cultivars Developed Through Molecular Breeding. New Delhi: Indian Council of Agricultural Research (2020). p. 52.

201. Carpentieri-Pipolo V. UEL 175: a novel lipoxygenase-free soybean cultivar with kunitz trypsin inhibitor absence. Crop Breed Appl Biotechnol. (2015) 15:191-2. doi: 10.1590/1984-70332015v15n3c33

202. Available online at: https://iisrindore.icar.gov.in/pdfdoc/NRC142News.pdf (accessed July 27, 2020).

203. Singh SP. Common Bean Improvement in the Twenty-First Century. Springer Science \& Business Media (2013).

204. Erskine W. Lessons for breeders from land races of lentil. Euphytica. (1997) 93:107-12. doi: 10.1023/A:1002939704321

205. Maranna S, Verma K, Talukdar A, Lal SK, Kumar A, Mukherjee K. Introgression of null allele of Kunitz trypsin inhibitor through markerassisted backcross breeding in soybean (Glycine max L. Merr.). BMC Genet. (2016) 17:106. doi: 10.1186/s12863-016-0413-2

206. Kumar V, Rani A, Rawal R, Mourya V. Marker assisted accelerated introgression of null allele of kunitz trypsin inhibitor in soybean. Breed Sci. (2015) 65:447-52. doi: 10.1270/jsbbs.65.447

207. Lyons G, Ortiz-Monasterio I, Stangoulis J, Graham R. Selenium concentration in wheat grain: is there sufficient genotypic variation to use in breeding? Plant Soil. (2005) 269:369-80. doi: 10.1007/s11104-0040909-9

208. Ye X, Al-Babili S, Klöti A, Zhang J, Lucca P, Beyer P, et al. Engineering the provitamin A ( $\beta$-carotene) biosynthetic pathway into (carotenoid-free) rice endosperm. Science. (2000) 287:303-5. doi: 10.1126/science.287.5451.303

209. Anacleto R, Badoni S, Parween S, Butardo Jr VM, Misra G, Cuevas $\mathrm{RP}$, et al. Integrating a genome-wide association study with a largescale transcriptome analysis to predict genetic regions influencing the glycaemic index and texture in rice. Plant Biotechnol J. (2019) 17:126175. doi: $10.1111 / \mathrm{pbi} .13051$

210. Wu G, Truksa M, Datla N, Vrinten P, Bauer J, Zank T, et al. Stepwise engineering to produce high yields of very long-chain polyunsaturated fatty acids in plants. Nat Biotechnol. (2005) 23:1013-7. doi: 10.1038/nbt1107

211. Ruiz-Lopez N, Haslam RP, Napier JA, Sayanova O. Successful high-level accumulation of fish oil omega-3 long-chain polyunsaturated fatty acids in a transgenic oilseed crop. Plant J. (2014) 77:198-208. doi: 10.1111/tpj.12378

212. Branham SE, Wright SJ, Reba A, Linder CR. Genome-wide association study of Arabidopsis thaliana identifies determinants of natural variation in seed oil composition. J Hered. (2016) 107:248-56. doi: 10.1093/jhered/esv100

213. Kim HJ, Silva JE, Vu HS, Mockaitis K, Nam J-W, Cahoon EB. Toward production of jet fuel functionality in oilseeds: identification of FatB acyl-acyl carrier protein thioesterases and evaluation of combinatorial expression strategies in Camelina seeds. J Exp Bot. (2015) 66:425165. doi: 10.1093/jxb/erv225

214. Nguyen HT, Park H, Koster KL, Cahoon RE, Nguyen HT, Shanklin J, et al. Redirection of metabolic flux for high levels of omega-7 monounsaturated fatty acid accumulation in camelina seeds. Plant Biotechnol J. (2015) 13:3850. doi: $10.1111 / \mathrm{pbi} .12233$

Conflict of Interest: The authors declare that the research was conducted in the absence of any commercial or financial relationships that could be construed as a potential conflict of interest.

Copyright (c) 2020 Gaikwad, Rani, Kumar, Gupta, Babu, Bainsla and Yadav. This is an open-access article distributed under the terms of the Creative Commons Attribution License (CC BY). The use, distribution or reproduction in other forums is permitted, provided the original author(s) and the copyright owner(s) are credited and that the original publication in this journal is cited, in accordance with accepted academic practice. No use, distribution or reproduction is permitted which does not comply with these terms. 